AFRL-SN-RS-TR-1999-250

Final Technical Report

December 1999

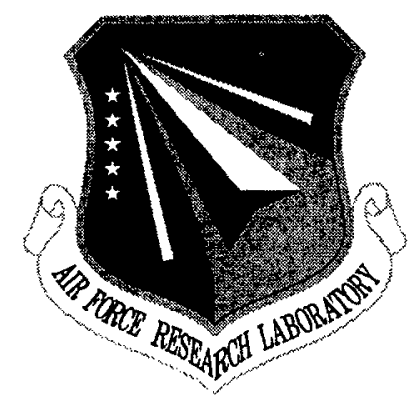

\title{
MULTICHANNEL RECEIVER
}

CHARACTERIZATION FOR ADAPTIVE ARRAY APPLICATIONS PHASE 5: EXPERIMENTS/DSP SOFTWARE

Eastern Shore Research Corporation

William L. Simkins

APPROVED FOR PUBLIC RELEASE; DISTRIBUTION UNLIMITED.

\section{5}

\author{
AIR FORCE RESEARCH LABORATORY \\ SENSORS DIRECTORATE \\ ROME RESEARCH SITE \\ ROME, NEW YORK
}


Although this report references limited documents (*), listed on page 81, no limited information has been extracted.

This report has been reviewed by the Air Force Research Laboratory, Information Directorate, Public Affairs Office (IFOIPA) and is releasable to the National Technical Information Service (NTIS). At NTIS it will be releasable to the general public, including foreign nations.

AFRL-SN-RS-TR-1999-250 has been reviewed and is approved for publication.

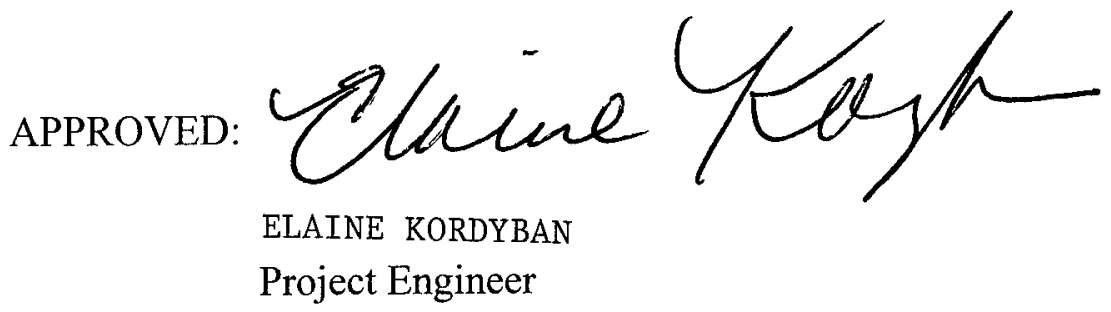

FOR THE DIRECTOR:

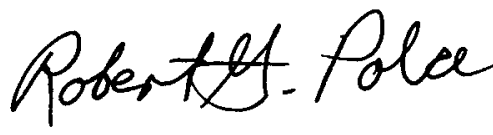

ROBERT G. POLCE, Acting Chief

Rome Operations Office

If your address has changed or if you wish to be removed from the Air Force Research Laboratory Rome Research Site mailing list, or if the addressee is no longer employed by your organization, please notify AFRL/SNRD, 26 Electronic Pky, Rome, NY 134414514. This will assist us in maintaining a current mailing list.

Do not return copies of this report unless contractual obligations or notices on a specific document require that it be returned. 


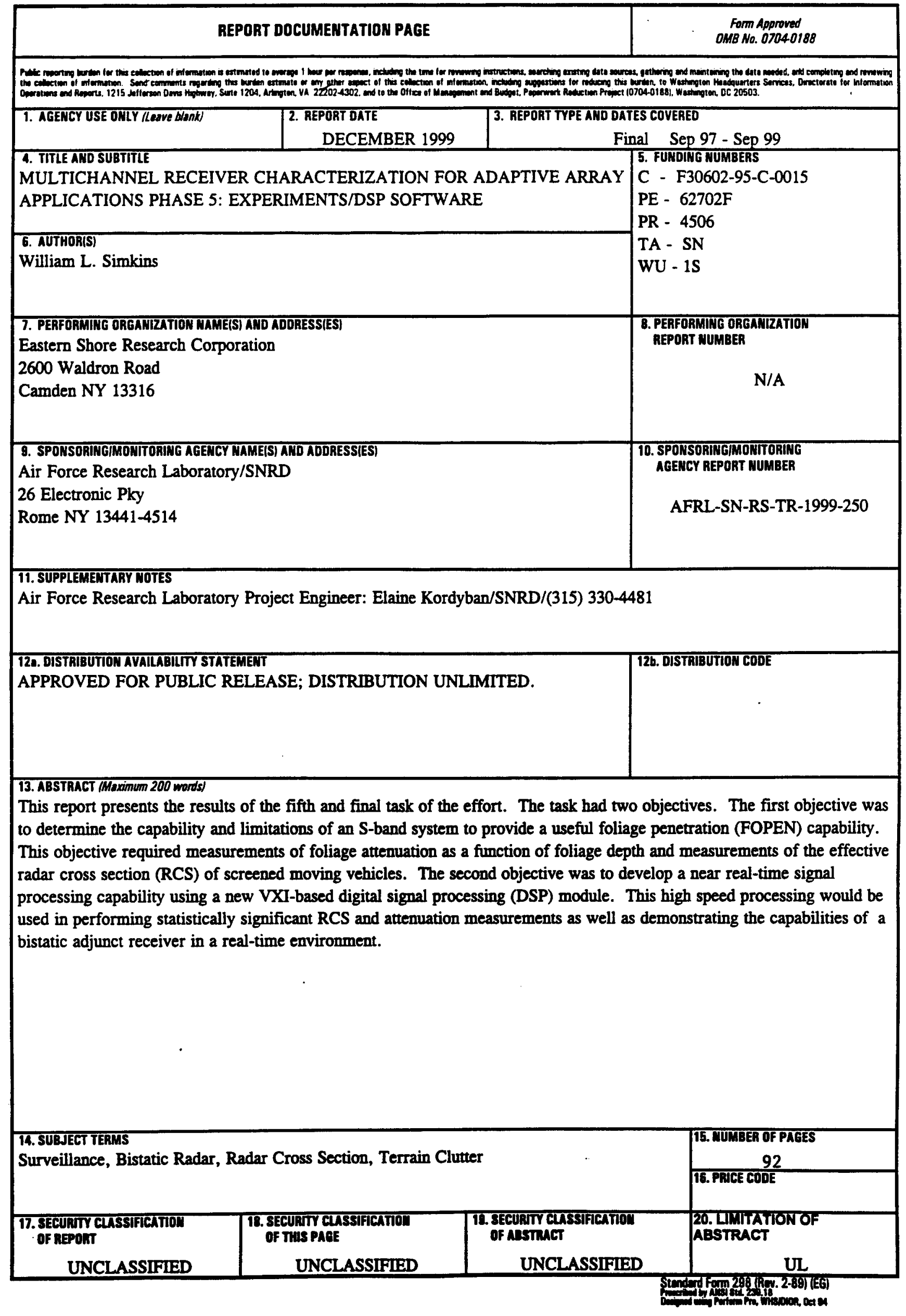




\section{TABLE OF CONTENTS}

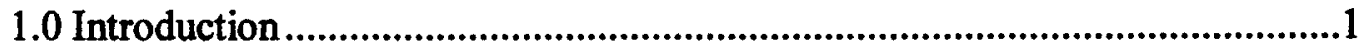

2.0 Review of the Bistatic Facility ..............................................................................

2.1 Waveform Generation and Up-Converter Subsystem...................................5

2.2 Optical and Transmitter Subsystem............................................................6

2.3 Antenna and Receiver Subsystem ..............................................................8

2.4 The A/D Converter, Digital and Timing Subsystem ...................................12

3.0 Attenuation of Trees and Foliage at S-Band Frequencies ..................................15

3.1 Attenuation Measurements ..........................................................................16

3.1.1 Town Barn Site ..................................................................................16

3.1.2 Screened Measurement Sites ................................................................. 21

3.1.2.1 Measurements at the Farrell Road Site..............................................22

3.2.2, Measurements at the Newport Road Site........................................29

4.0 Moving Target Experiments ............................................................................31

4.1 Test Setup and Procedures.................................................................................31

4.2 Vehicle Measurements at the Town Barn Site..............................................31

4.3 Vehicle Measurements at the Farren Road Site 34

$\$ .4$ Vehicle Measurements at the Newport Road Site .........................................38

5.0 VXI DSP Processing Subsystem ....................................................................40

5.1 LeMans VX8 Carrier Board .......................................................................42 
5.1.2 DSP Software Development Tools ........................................50

5.1.2.1 VX8 Software Libraries ..............................................50

5.1.2.2 TI TMS320C3x/C4x Code Generation Tools .....................52

5.1.2.2. GO DSP Code Composer............................................53

5.1.2.3. Mountain-510 Universal DSP Emulator- PC-AT/ISA .........54

5.1.2.4 Sinectoanalysis Math Libraries........................................54

5.2 C4x DSP Signal Processing Software ............................................55

5.2.1 Nodes C and D - Digital I/Q Demodulation ............................57

5.2.2 Nodes C1 and D1 - Range-Doppler Processing ..........................62

5.2.3 Node B - Digital Antenna Beamforming .................................66

5.2.4 Node A - Host Interface and Interrupt Servicing.........................70

5.2.5 Data Transfer and System Thruput ...........................................71

5.3 Lessons Learned .........................................................................74

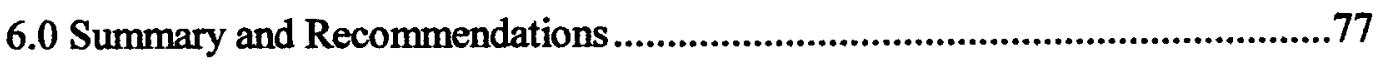

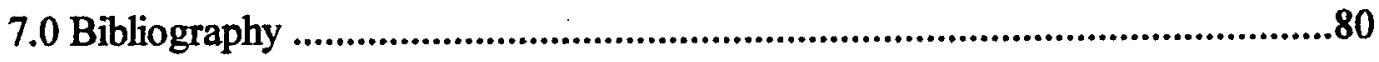

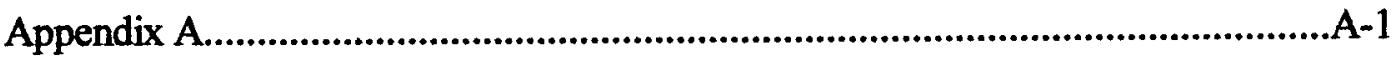

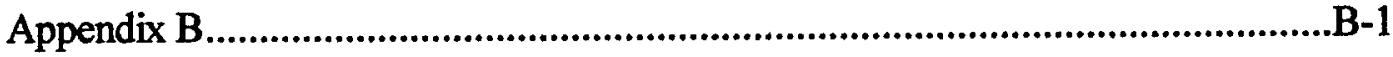




\section{LIST OF FIGURES}

Figure 2.1 Block diagram of bistatic receiver (August, 1996) ...............................4

Figure 2.2 User console with computer display, AWG and test equipment............5

Figure 2.3 Up-converter block diagram (October 1995) .......................................6

Figure 2.4 Optical transmitter.....................................................................................7

Figure 2.5 S-Band antenna (front view) ..............................................................8

Figure 2.6 S-Band antenna (rear view) .................................................................8

Figure 2.7 S-Band antenna with radome .................................................................9

Figure 2.8 S-Band preamp and first mixer ........................................................10

Figure 2.9 IF subsystem ...................................................................................10

Figure 2.10 SAW IF filter response .......................................................................11

Figure 2.11 LO chain ..........................................................................................12

Figure 3.1 Topographic map of town barn reference site .......................................17

Figure 3.2 Radar display of calibrator signal over the topographic map.................18

Figure 3.3 Aerial orthophoto view of the Newport Road and Farrell Road sites...20

Figure 3.4 View of Newport Road and Farrell Road reference sites

from the bistatic receiver ............................................................................. 21

Figure 3.5 View toward the bistatic transmitter and receiver

from Farrell Road reference site ........................................................ 22

Figure 3.6 Screening vegetation toward receiver at $100 \mathrm{ft}$ location .......................24

Figure 3.7 Screening vegetation in direction of receiver at $200 \mathrm{ft}$ location.............24

Figure 3.8 Screening vegetation in direction of receiver at $400 \mathrm{ft}$ location.............24

Figure 3.9 Range-Doppler display of calibrator reference signal............................24 
Figure 3.10 Foliage attenuation measurements as a function of location. .28

Figure 3.11 Foliage attenuation measurements as a function of vegetation depth .28

Figure 4.1 Topographic map of town barn reference site .32

Figure 4.2 Processed signal from the test vehicle near the Town Barn site .32

Figure 4.3 Range-Doppler-RCS map of auto traveling away from radar .33

Figure 4.4 Power and effective RCS from vehicle

in filter 7 of 16 . (Auto 0,6/24/98) 34

Figure 4.5 Range Doppler display of vehicle return

at the reference site. (Auto 0,8/19/98) .35

Figure 4.6 Range-Doppler display of vehicle return at $200 \mathrm{ft}$ west. (Auto 200, 8/19/98) .35

Figure 4.7 Effective vehicle RCS as a function site location .37

Figure 4.8 Effective vehicle RCS as a function of foliage depth .37

Figure 4.9 The power and effective radar cross section from the test vehicle screened by the canopy of a tree .38

Figure 4.10 Range-Doppler display of vehicle RCS and unknown interference.....38

Figure 5.1 LeMans VX8 Bus Architecture 41

Figure 5.2 System Definition (SDF) created for the existing VX8 Board ..............43

Figure 5.3 Embedded Node A architecture .....................................................46

Figure 5.4 MDC44ST Block Diagram .........................................................47

Figure 5.5 Comm port routing................................................................48

Figure 5.6 Example of an Load Definition File for the existing VX8 system..........51 
Figure 5.8 Code Composer Graphic Interface......................................................53

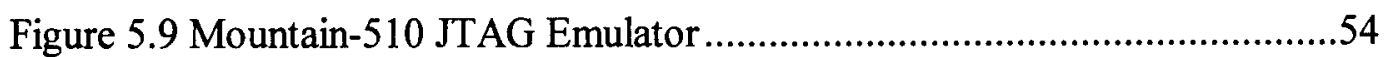

Figure 5.10 Block Diagram of Signal Processing Distribution

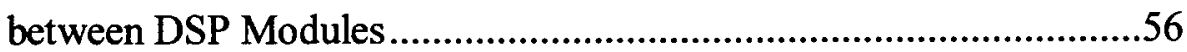

Figure 5.11 Structure of the Pipeline Parallel Processing Approach ......................56

Figure 5.12 Command File for Nodes $\mathrm{C}$ and $\mathrm{D}$, processors 0 and $1 \ldots \ldots \ldots \ldots \ldots \ldots . . . . .58$

Figure 5.13 Preprocessing and Digital I/Q processing (Node C0/D0)..................61

Figure 5.14 Channel Compensation and Range-Doppler Processing....................64

Figure 5.15 Beamforming Processing (Node B)..................................................68 


\section{LIST OF TABLES}

Table 2.1 Measured amplitude loss in calibration cable ..........................................9

Table 2.2 Specifications of the HP E1429A/B $20 \mathrm{MSa} / \mathrm{s} 2$-channel digitizers........14

Table 3.1 Median attenuation through a forest canopy in Maine ..........................15

Table 3.2 Town barn calibration measurements .......................................................18

Table 3.3 One way attenuation measurements at the Farrell Road site...................26

Table 3.4 Bistatic attenuation measurements at Farrell Road site ...........................27

Table 3.5 Newport Road Two-Way Attenuation Measurements ............................30

Table 4.1 Summary of Vehicle Measurements at Town Barn site...........................33

Table 4.2 Summary of Screened Vehicle Measurements at Farrell Road ...............36

Table 4.3 Summary of Screened Vehicle Measurements

at Newport road (site C) ...........................................................................39

Table 5.1 VX8 Timing Cycles .............................................................................49

Table 5.2 Timing Profile for Node C0 Processing per 64K record ........................62

Table 5.3 Timing Profile for Node C1/D1 Processing per 64K record..................67

Table 5.4 Timing Profile for Node B Processing per 64K record .........................69

Table 5.5 Total System Timing per 64K Record ....................................................73 


\subsection{INTRODUCTION}

The Department of Defense has an interest in the detection of low visibility threats. One approach, currently under investigation by the Air Force Research Laboratory (AFRL), involves the development of Advanced Offboard Bistatic Technology for improved detection and tracking of low visibility targets. For the purpose of this report, low visibility targets are those with an inherently low radar cross section (RCS) or those that use natural features to reduce or mask the targets return. Stealth technology is an example of the first type while the aircraft using terrain shadowing or vehicles using the attenuation and clutter of a vegetation canopy are examples of the second type. The sensor technology involves the development of a modern adaptive multichannel bistatic radar system for use with cooperative and non-cooperative transmitters.

One goal of an adaptive ground-based or airborne surveillance radar system is to have optimum or near-optimum detection and tracking of weak targets in the presence of strong clutter and interference while maintaining a low false alarm rate. Several adaptive techniques have been suggested to meet these criteria including adaptive space-time processing [1,2], adaptive multipath and jamming cancellation $[3,4]$ and adaptive beamforming [5]. The performance of all these techniques depends on the target-to-noise ratios (T/N), the clutter-plus-interference-tonoise ratios $(\mathrm{C}+\mathrm{I} / \mathrm{N})$ and the spatial-temporal amplitude and correlation statistics of the target, the clutter and the interference. Radar measurements and experiments are required to demonstrate the performance of these techniques and to quantify the target and clutter statistics that determined each technique's limitations.

The objective of this effort is to assist the Air Force Research Laboratory in creating a fundamental multichannel measurement capability to perform multidomain adaptive radar experiments. A multi-phase effort was performed to assist AFRL in the development of the existing multichannel receiver into a multi-use bistatic testbed. The first phase report [6] provided an evaluation of the existing system with recommendations for improving performance. The second phase recommended the procedures and post- $A / D$ algorithms for maintaining real-time calibration of the adaptive array receiver [7]. The third task presented an assessment of the existing adaptive array receiver's utility for investigating clutter phenomenology and defined two experiments that demonstrate the capabilities of AFRL's adaptive receiver's capabilities [8]. The fourth task developed procedures for using the calibrated transponder in the radar cross section measurements of clutter and targets and demonstrated these procedures in the measurement of the local clutter environment [9]. 
This report presents the results of the fifth and final task of this effort. This task had two objectives. The first objective was to determine the capability and limitations of an S-Band system to provide a useful foliage penetration (FOPEN) capability. This objective required measurements of foliage attenuation as a function of foliage depth and measurements of the effective RCS of screened moving vehicles. The second objective was to develop a near real-time signal processing capability using a new VXI-based DSP module. This high speed processing would be used in performing statistically significant RCS and attenuation measurements as well as demonstrating the capabilities of a bistatic adjunct receiver in a real-time environment.

Chapter 2.0 presents a brief review of the existing bistatic array receiver and its auxiliary equipment. The discussion documents the changes and upgrades that have occurred since 1995 and describes the system that was used to collect the data presented in this report. Chapter 3.0 presents the foliage attenuation measurements at S-Band frequencies. Chapter 4.0 presents the results of some screened target detection experiments. Chapter 5.0 presents a description of the LeMans VX8 DSP module and its associated software. This section presents the radar processing developed for use in measurements or as a bistatic adjunct receiver. Timing profiles are presented to show that the current near real-time processing can provide real-time demonstrations when used with many current monostatic radars. Chapter 6.0 presents a summary of the effort's results and recommendations for future projects and system upgrades. 


\subsection{REVIEW OF THE BISTATIC FACILITY}

This section provides a brief overview of the bistatic system as it has developed from 1995 through 1998. Figure 2.1 shows the configuration of the Newport testbed during 1998. The system consists of several subsystems for waveform generation, control and data collection. A standard Pentium-based personal computer (PC) operating under the WinNT operating system provides the user interface and provides a long-term storage system for data. The PC also controls the VXI data collection system via a HP E1482B VXIbus Extender and a HP E1489C ISA-to-MXIbus Interface. The Arbitrary Waveform Generator (AWG) provides a programmed waveform on a $5 \mathrm{MHz}$ carrier to the up-converter. The output of the upconverter is manually transferred to either a calibration port for internal calibration or to the optical transmitter. The optical signal is received and demodulated at the transmitter site on Tanner Hill. This S-Band signal is provided to either the low power transmitter for array compensation measurements or to the high power transmitter for most experiments. The output of the bistatic receiver array is down-converted to a $5 \mathrm{MHz}$ carrier, digitized and stored in an internal high speed buffer memory. The data is then transferred to the DOS-based storage in the controller. System coherence is maintained in the converter oscillators and the digitizers by locking all clocks to a $10 \mathrm{MHz}$ reference.

A second optical system for remote control purposes was under development and was not used thru 1998. Also shown is an auxiliary receiver using a 4 foot dish and a log amplifier. This subsystem is used during synchronization when the bistatic system is used with another host transmitter.

In 1997, the high power transmitter subsystem was moved to a location on Irish Hill. The 10 foot dish and pedestal were placed in a concrete pad on the northeast side of the main building with the transmitter placed in a nearby location within the building. The low power $\mathrm{CW}$ transmitter remained on Tanner Hill and used a sheltered 10 foot dish to provide a calibration signal. Use of the low power and high power transmitter required manually switching the optical output from the receiver at the optical junction box. 


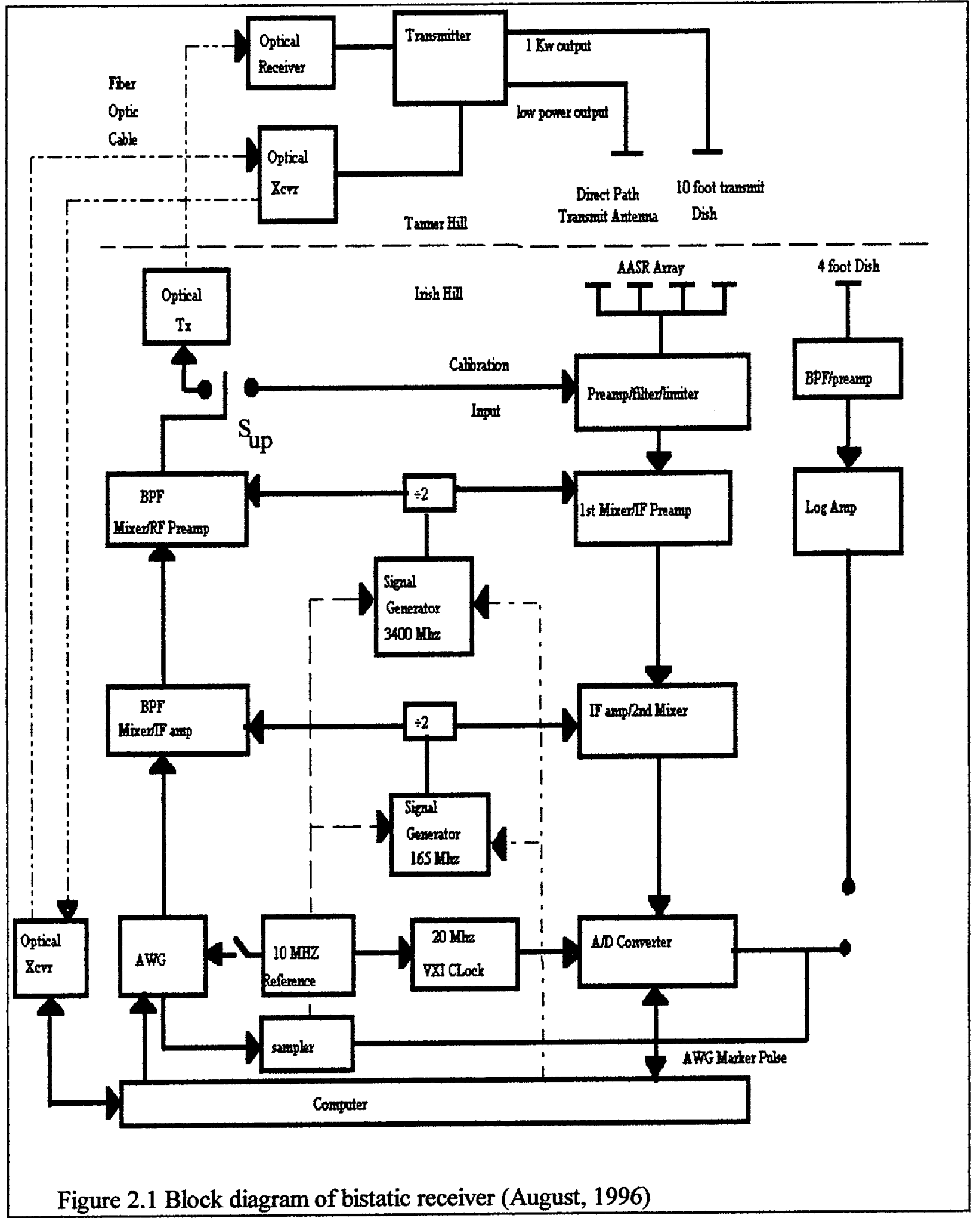




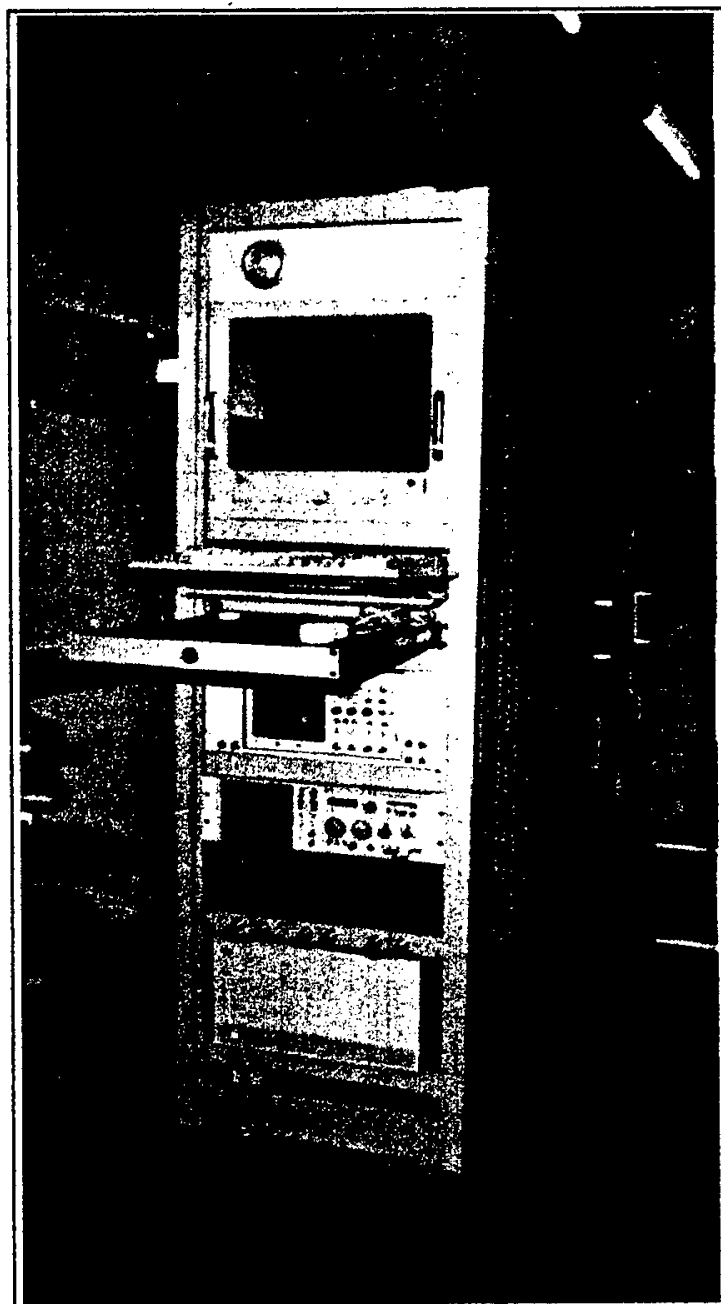

Figure 2.2 User console with computer display, AWG and test equipment

\subsection{Waveform Generation and Up-Converter}

Subsystem

The waveform generation and up-converter subsystem provides calibration waveforms as well as radar transmitter signals for use at the Newport site. The waveform is generated using a HP $8770 \mathrm{~A}$ Arbitrary Waveform Generator (AWG), a programmable device that provides waveforms with frequencies up to $50 \mathrm{MHz}$ via a high-speed 125 $\mathrm{MHz}, 12$ bit Digital-to-Analog Converter (D/A). Figure 2.2 shows the user console that houses the AWG in the lower shelf and the user console and test equipment in the higher shelves. The high dynamic range and high degree of oversampling allow the AWG to provide a high quality signal centered at 5 $\mathrm{MHz}$. The manufacturer [10] sites phase linearity of $+/-5$ degrees, harmonic distortion less that $50 \mathrm{dBc}$ and single-sideband (SSB) phase noise of less than $120 \mathrm{dBc}$ at a $10 \mathrm{kHz}$ offset. Within the $2.5 \mathrm{MHz}$ to 7.5 $\mathrm{MHz}$ passband of the receiver, the signal-tophase-noise ratio $(\mathrm{S} / \mathrm{N})$ at the $A W G$ output is approximately $60 \mathrm{~dB}$. The AWG is programmed to provide different waveforms via a software interface developed by Rome Research, Inc. System coherence is maintained by phase-locking the AWG's internal sampling clock to the system's $10 \mathrm{MHz}$ reference oscillator.

The AWG signals are up-converted to S-Band using the same LO sources as those used in the receiver. Figure 2.3 presents a block diagram of the up-converter fabricated by Rome Lab personnel. The noise contributed by the up-converter components include both thermal and phase noise components. The $\mathrm{S} / \mathrm{N}$ limitation of the thermal noise components is approximately $80 \mathrm{~dB}$, over $10 \mathrm{~dB}$ below the AWG noise level, and does not significantly impact the test signal's quality.

The primary phase noise contributors are the oscillator used to create the signal, the oscillator used to provide the A/D sampling clock and the two local oscillators used in frequency conversion. When the same oscillators are used in both the transmitter up-conversion and receiver 
down-conversion, the phase noise power is a function of the short-term stability of these oscillators and the time delay $t_{d}$ between transmission and reception. The power in the phase noise sidebands $S(f)$ and the total phase noise $N_{\text {phase }}$ can be given as [11,12]

$$
\begin{aligned}
& S\left(f_{m}\right)=4\left(\frac{\Delta f}{f_{m}}\right)^{2}\left[\sin \left(\pi f_{m} t_{d}\right)\right]^{2} \\
& N_{\text {phase }}=\int_{0}^{B_{c a t}} S\left(f_{m}\right) d f_{m}
\end{aligned}
$$

where $f_{m}$ is the offset frequency of the phase modulation, $\Delta f$ is the frequency deviation in $\mathrm{Hz}$ and $B_{c o h}$ is the noise bandwidth of the receiver after coherent processing. For stable crystal oscillators, the fractional frequency deviation $\left(\Delta \mathrm{f} / \mathrm{f}_{\mathrm{m}}\right)$ is typically less that $10^{-5}$ at $\mathrm{f}_{\mathrm{m}}=10 \mathrm{kHz}$ dropping to a floor of less than $10^{-7}$ at $f_{m}=100 \mathrm{kHz}$ and higher offset frequencies. For the calibration measurements discussed in the next section, the time delay through the up-converter, calibration cables and the receiver is approximately 4 usec. For a noise bandwidth of $5.6 \mathrm{MHz}$, this results in a phase noise power of less than $60 \mathrm{~dB}$ below the carrier. With the stable VHF and microwave oscillators used in the system, the phase noise corresponding to such a small time delay is insignificant. However, at longer ranges or when another transmitter source is used, phase noise will become more important.

\subsection{Optical and Transmitter Subsystem}

The Newport test site consists of two hilltop sites that are approximately 6600 feet apart and 330 feet above the intervening valley. Optical fiber cable is available for the transmission of

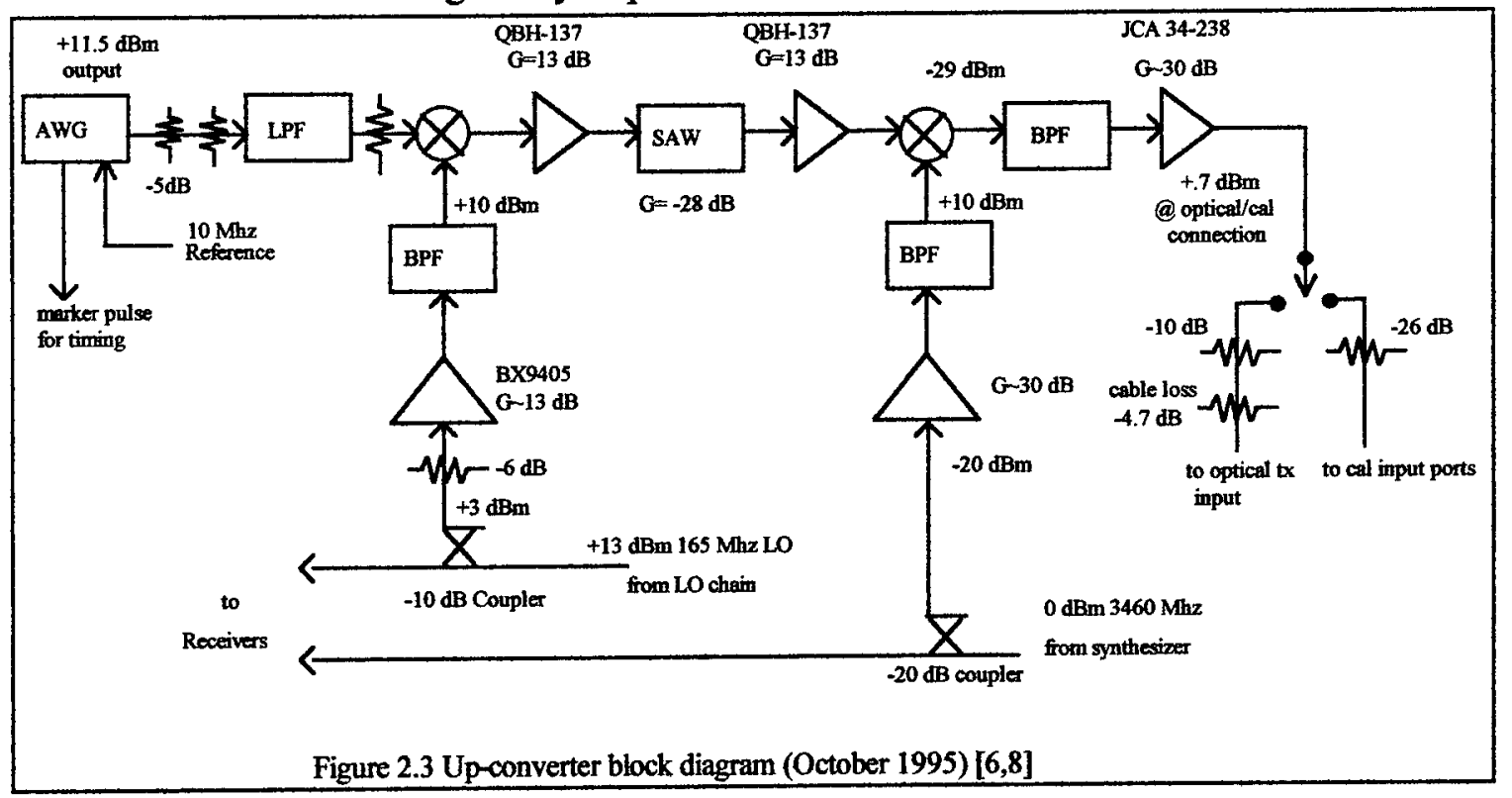




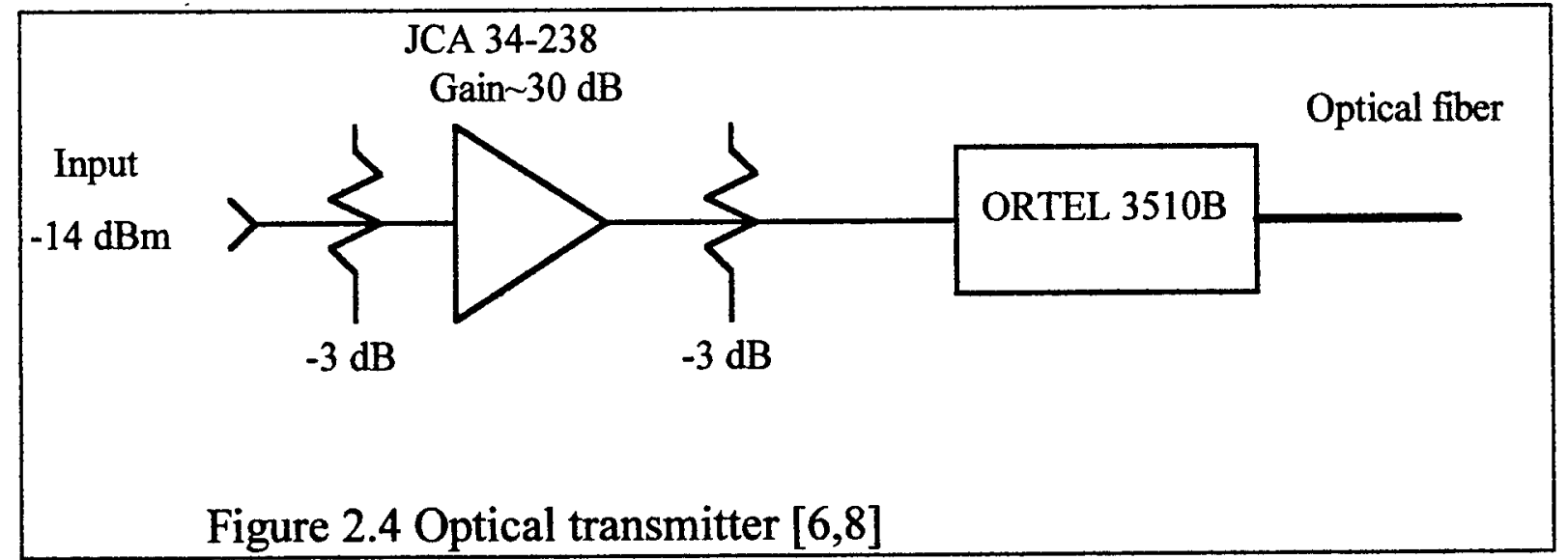

timing and RF signals between the Tanner Hill and Irish Hill sites. The bistatic testbed shelter is located on Irish Hill and uses this optical link to send transmit signals to a TWT transmitter located on Tanner Hill. The optical transceiver system consists of an Ortel 3510B optical transmitter and an Ortel 4508 optical receiver. As of 1997, a second optical transceiver system was to be installed to provide timing and remote control of the recently installed $1 \mathrm{~kW}$ (peak) pulsed transmitter and to allow remote monitoring of the parameters of the pulse transmitter, $\mathrm{cw}$ transmitter and the transmit antenna.

At S-Band, the maximum linear signal is obtained at the optical receiver with an input of $+10 \mathrm{dBm}$ into the optical transmitter. The loss in the optical system is approximately $-52 \mathrm{~dB}$ and the measured signal-to-noise ratio $(\mathrm{S} / \mathrm{N})$ ratio at the output is approximately $45 \mathrm{~dB}$. The measurement of optical loss is highly dependent on the quality and cleanliness of the optical cable connectors. Repeated removals and reinsertions of the fiber cable can easily provide several $\mathrm{dB}$ of change in the observed optical loss and $\mathrm{S} / \mathrm{N}$.

The third-order intercept point of the optical system is listed at over $+25 \mathrm{dBm}$. This is not important for typical radar waveforms such as gated CW, pseudorandom phase codes and LFM, because such waveforms provide only one frequency at a given instant in time. Such waveforms can be transmitted at levels as high as $+10 \mathrm{dBm}$ input with good fidelity. However, tests using the simultaneous transmissions of multiple frequency waveforms require a compromise in total input power and fidelity.

Both low power ( $<25$ watts) and high power pulsed TWT's have been discussed for use in future experiments. The low power transmitter is used primarily with a fixed antenna for receiver calibration and equalization. The $1 \mathrm{~kW}$ (peak) pulsed TWT uses a steerable 10 foot dish to provide signals for local experiments. 


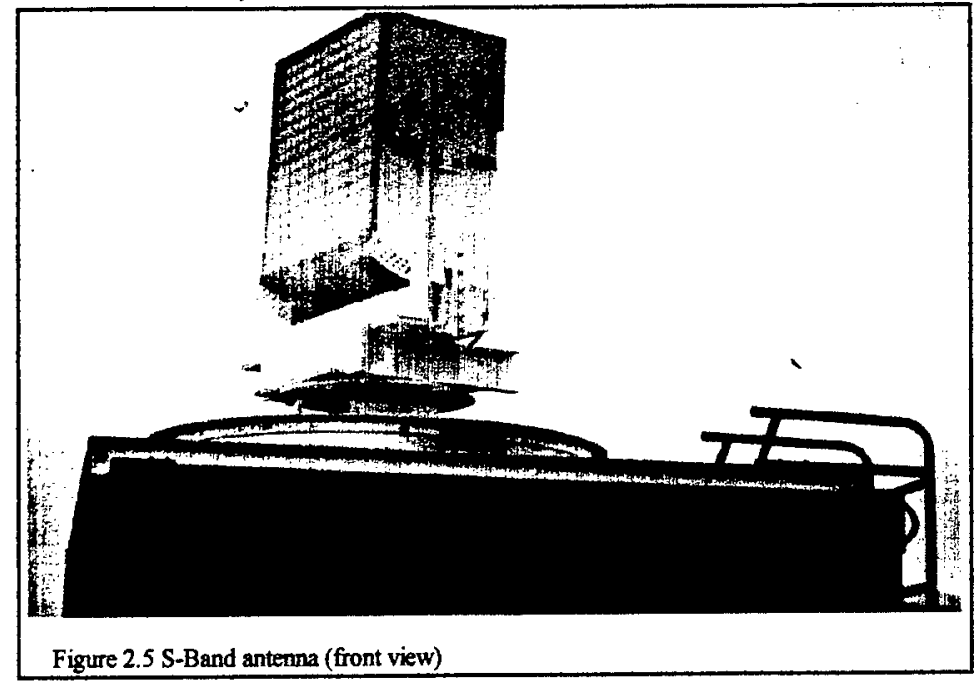

2.3 Antenna and Receiver Subsystem

Figures 2.5 and 2.6 show the front and rear view of the antenna respectively while Figure 2.7 shows the antenna with the radome in place. The antenna is a passive device consisting of 16 columns, each column consisting of 16 patch stripline antennas coupled with stripline couplers. The antenna is mounted on a

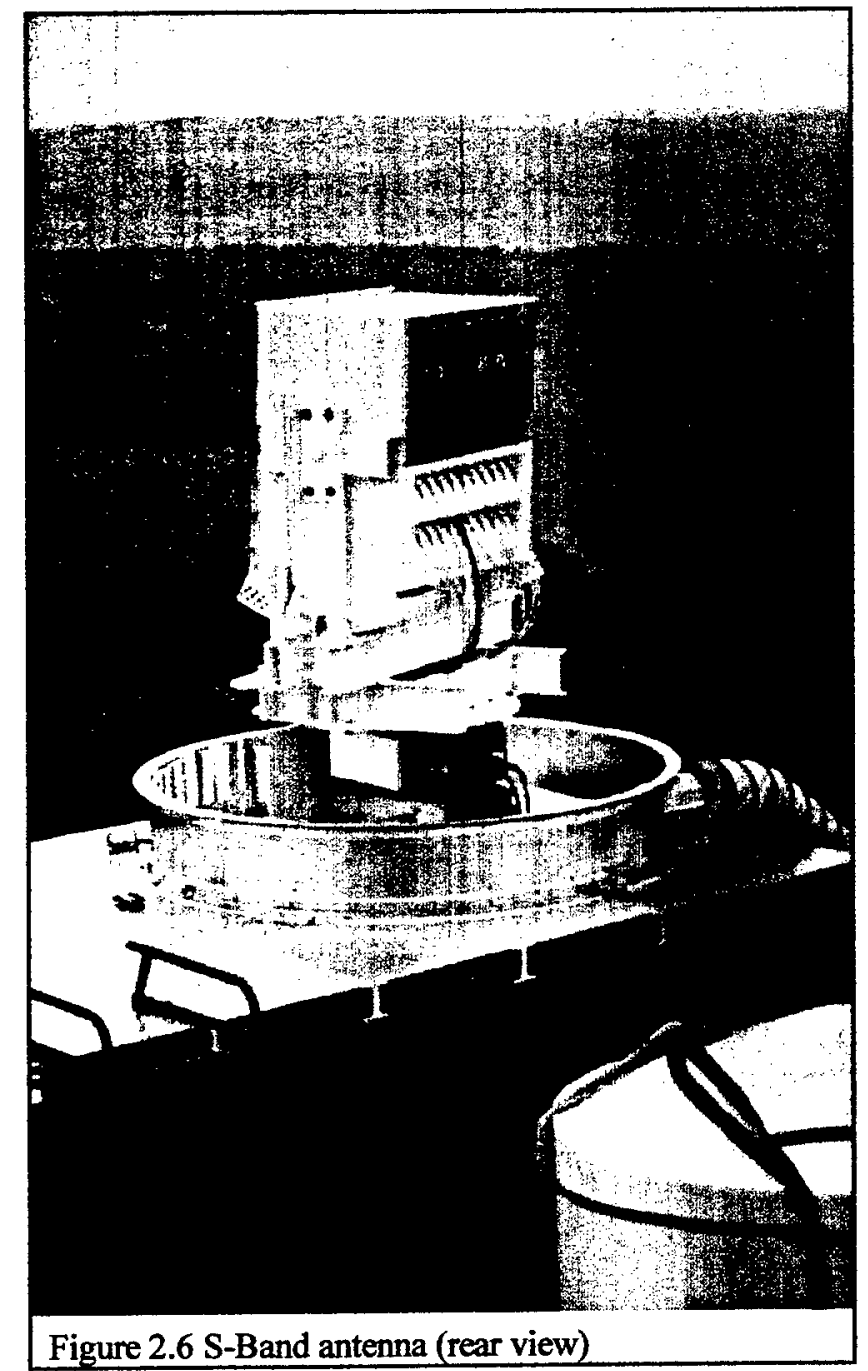
pedestal that provides digital-controlled azimuth steering of plus or minus 180 degrees. The elevation angle is positioned manually from -8 degrees to +8 degrees. In 1996, the front third of the radome plastic was replaced with clear Lexan to reduce loss.

Figure 2.8 shows a block diagram of the preamp assembly and the first mixer located in the receiver. The $R F$ preamp modules are mounted near the column outputs to reduce the line lengths and the associated losses contributing to system noise figure. The hardline cables used between the antenna and the preamp assembly were carefully matched to preserve the gain and phase matching of the array outputs. Adjustable lines are also included to allow compensation of the phase differences in the preamp assemblies.

The preamp assembly's limiter is used to prevent damaging signal levels into the receiver while an SPDT switch provides further protection and reduces the 


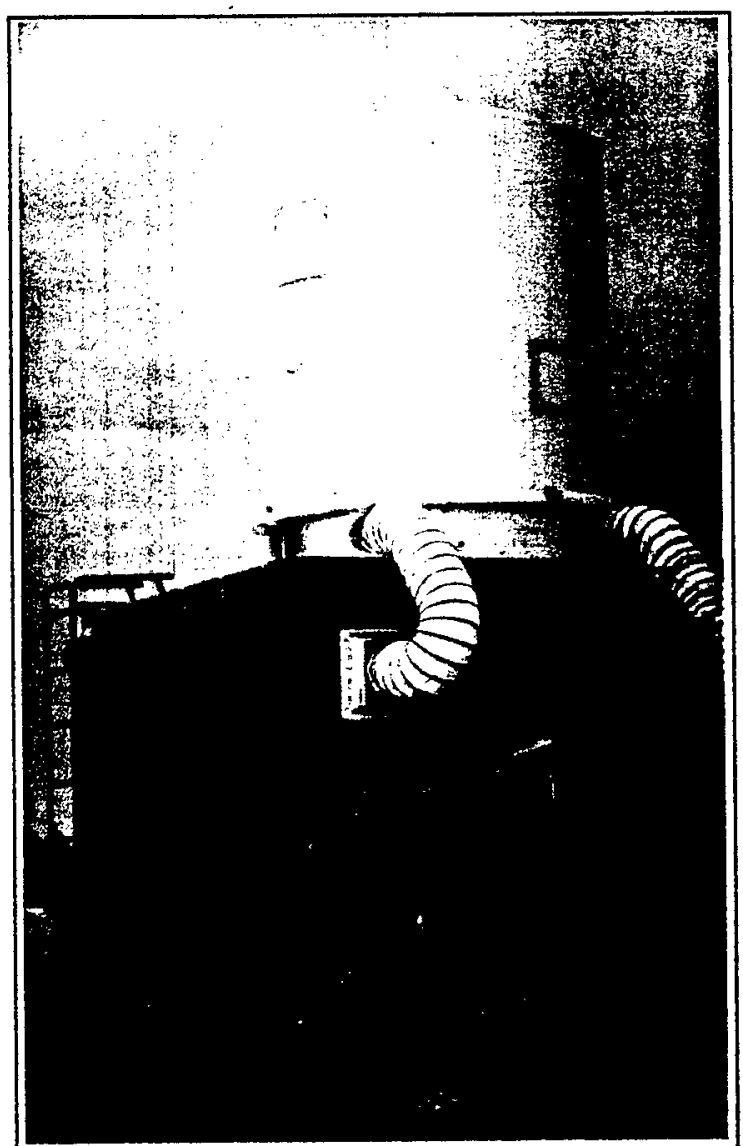

Figure 2.7 S-Band antenna with radome interference from external signals during calibration tests. The $-10 \mathrm{~dB}$ coupler allows the injection of a known signal for calibration and provides the test outputs used to form uniformly weighted sum and difference channels for setup and diagnostics.

When the system is in the calibration mode, the calibration signal is fed via hardline from the shelter to the antenna compartment where it is split into 16 channels, each channel associated with a column receiver. The measured loss and phase shift from the optical/cal switch to the coupler input are given in Table 2.1. The nominal loss is $18.2 \mathrm{~dB}$ at $3300 \mathrm{MHz}$ with a $(-.2 /+.1 \mathrm{~dB})$ variation from column to column. The variation over the $5 \mathrm{MHz}$ band is within $+/-.2 \mathrm{~dB}$. The peak deviation of the phase from linear was less than 1 degree for each column.

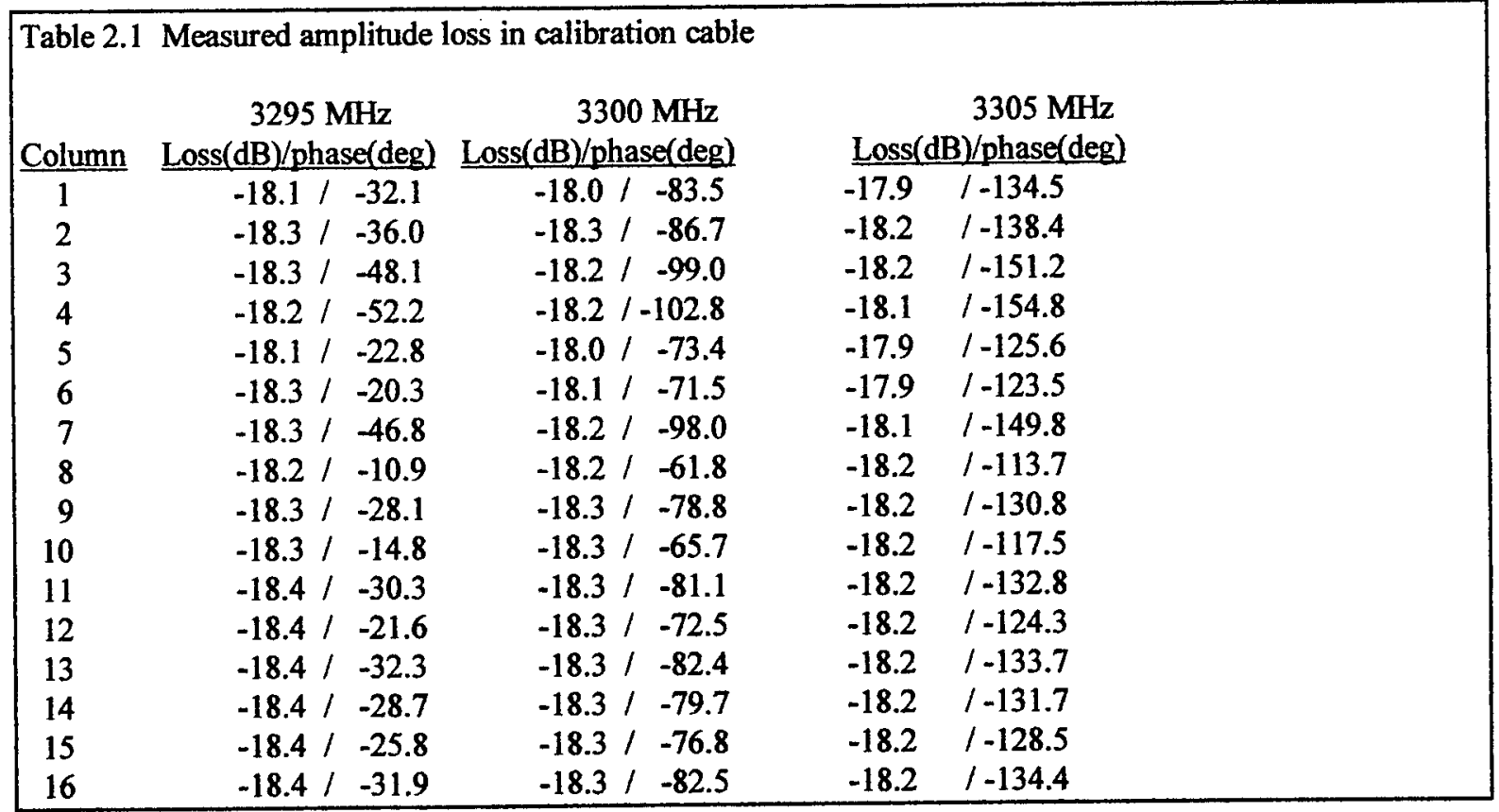




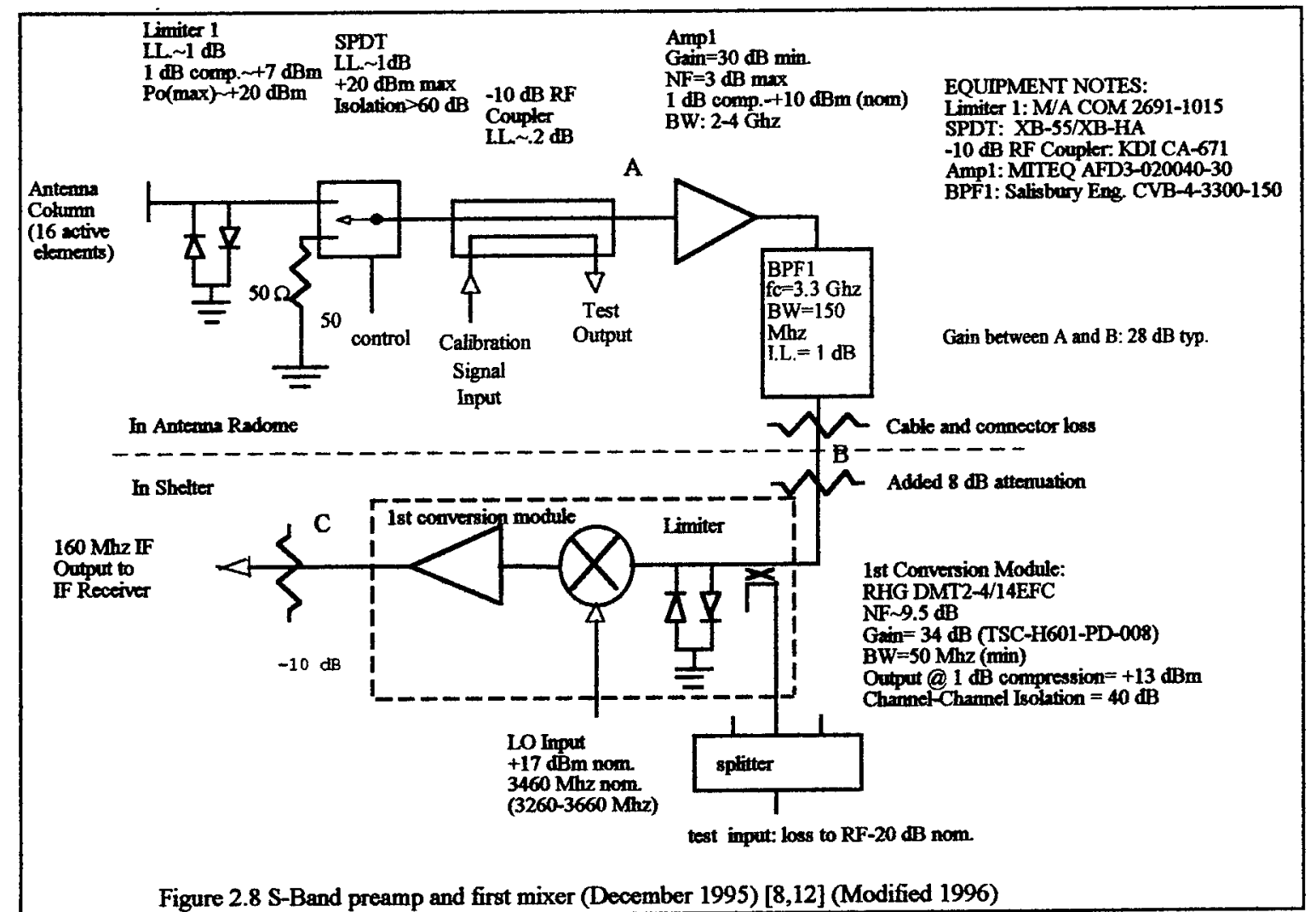

Figure 2.8 S-Band preamp and first mixer (December 1995) $[8,12]$ (Modified 1996)

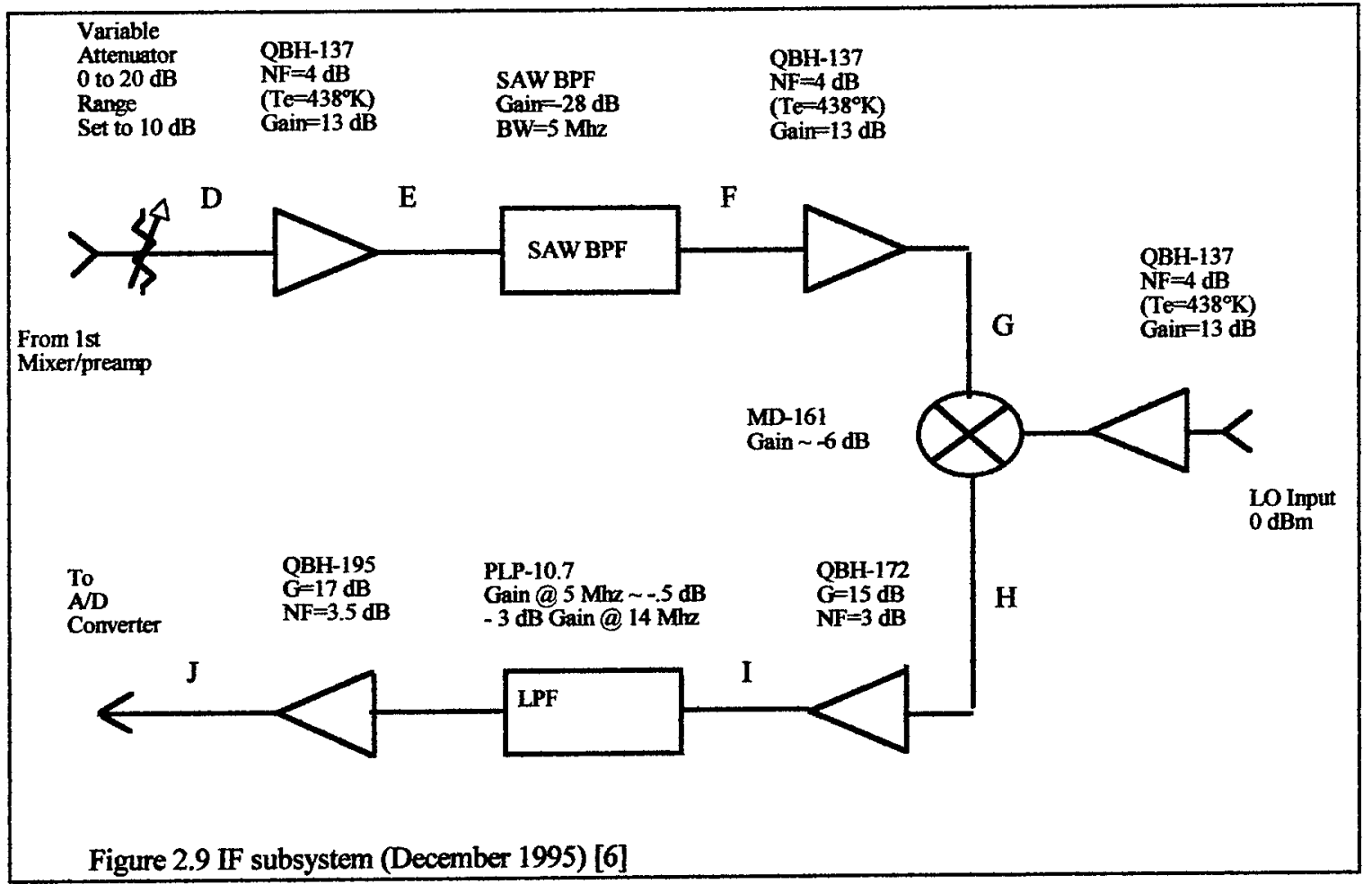


The preamps have a nominal noise figure of 3 $\mathrm{dB}$ and a minimum gain of $30 \mathrm{~dB}$. The 4-pole bandpass filter has a bandwidth of $150 \mathrm{MHz}$ to reduce the system's response to the image signals and the out-of-band interference. Matched lengths of hardline cable are used to transfer the 16 column signals from the bandpass filters located in the preamp assembly to the receivers located within the shelter.

An earlier interim document [6] characterized the receiver and made recommendations for improving the system's linear dynamic range. For a modest increase in noise figure $(.85 \mathrm{~dB})$, the linear

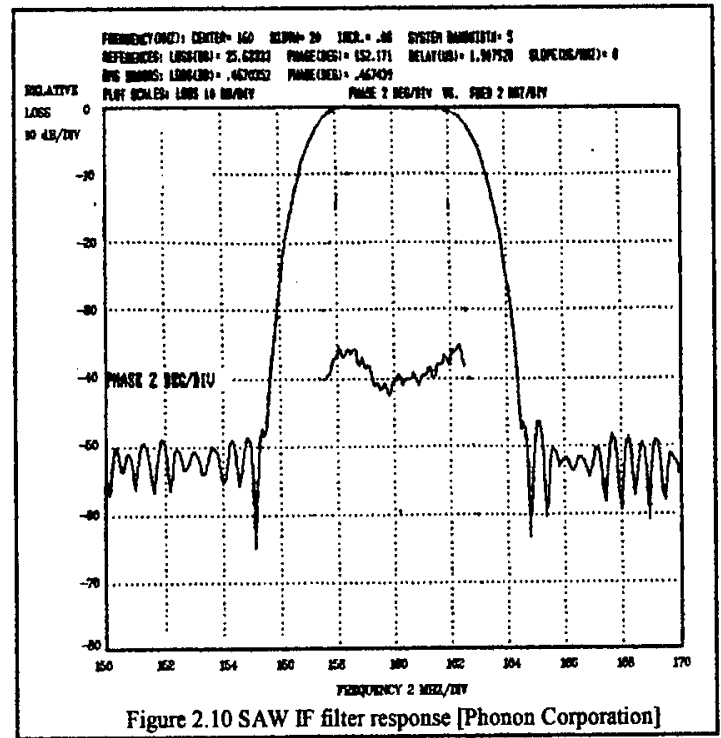
dynamic range for third order distortion could be increased close to the limitation provided by the "10 "effective bits" advertized for the 11 bit A/D converter [13]. Since the system is used where the experimental parameters can be chosen to meet the $\mathrm{S} / \mathrm{N}$ limitations and where the linear dynamic range is important, then the trade-off between linear dynamic range and noise figure is reasonable.

As shown in Figure 2.9, the receiver gain was reduced by $8 \mathrm{~dB}$ and the nominal linear dynamic range of the receivers was measured to be $66 \mathrm{~dB}$ [7]. The figure also presents a block diagram of the IF receiver. Most of the receiver's selectivity is provide by the surface acoustic wave (SAW) bandpass filter. Figure 2.10 presents the filter shape and general characteristics of these devices. The $-3 \mathrm{~dB}$ bandwidth is nominally $5.6 \mathrm{MHz}$ and a nominal rejection between -45 and $-50 \mathrm{~dB}$ is obtained at offset frequencies from $4.8 \mathrm{MHz}$ to $80 \mathrm{MHz}$. The Mini-Circuits PLP10.7 low-pass filter provides additional selectivity, reducing adjacent channel signals and noise beyond $15 \mathrm{MHz}$.

The isolation between channels is limited by the LO chain (Figure 2.11). The RHG 3channel mixer/preamps specify a $40 \mathrm{~dB}$ minimum isolation between the three channels contained within each module. The circulator and splitter provides an additional $40 \mathrm{~dB}$ of isolation between different modules. The 8-way splitter and the MD-161 mixers used in the second down conversion provide a minimum of $50 \mathrm{~dB}$ isolation. 


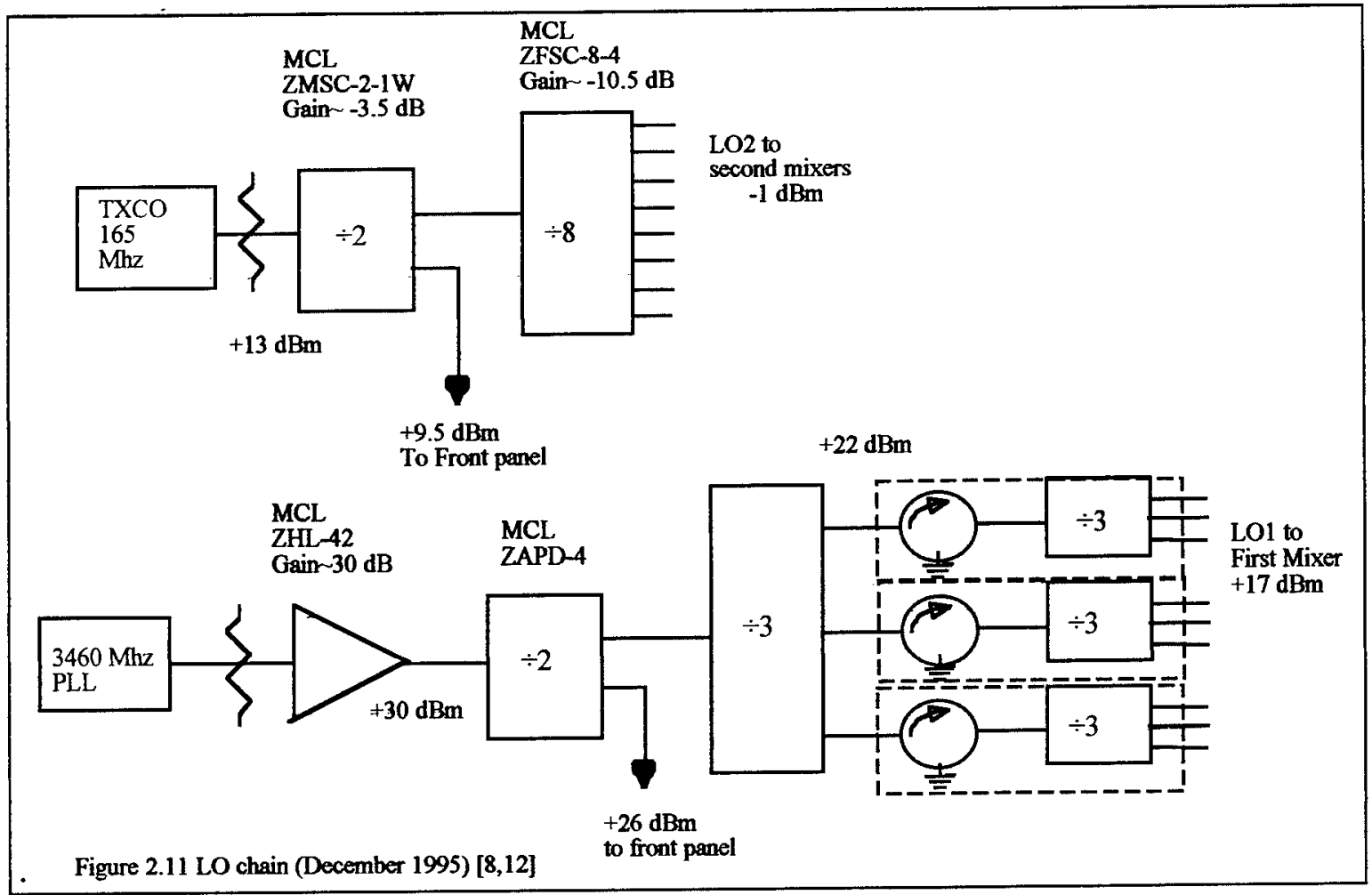

\subsection{The A/D Converter, Digital and Timing subsystems}

The original digital subsystem is a VXI-based system consisting of a Radisys 486-based controller, 8 dual-channel analog-to-digital (A/D) converters, VXI-based hard drives and an interface for logging GPS time. Each A/D converter is an HP E11429B digitizer, a dual-channel 12-bit VXI-based device capable of performing 20 million sample/second. Table 2.2 lists some of the specifications of these dual-channel converters. The harmonic distortion, at $61 \mathrm{~dB}$ down from a full scale signal, and the total noise and distortion of the $\mathrm{A} / \mathrm{D}$ converters from all sources, at 59 $\mathrm{dB}$ down, are less than that created by the receiver's final amplifier. The timing of the bistatic receiver consists of several oscillators used for frequency conversion, A/D conversion and other timing. Coherence is maintained by locking the oscillators to a $10 \mathrm{MHz}$ reference.

The manufacturer claims that the 1 volt, $50 \mathrm{ohm}$ single-ended range provides the most linear A/D performance. The Least Significant Bit (LSB) signal specifies a sinusoidal signal at the $\mathrm{A} / \mathrm{D}$ converter with an $\mathrm{rms}$ value equal to the LSB. For an 12 bit A/D converter operated in its +/1 volt bipolar mode ( 11 bits plus sign), the digital output has a range from +2046 for a 1.023 volt peak input to -2045 for a -1.0225 volt peak input. (-2048, -2047, -2046 and 2047 either indicate overload or are not used.) The LSB corresponds to $1.023 / 2047=.0005$ volts. An LSB signal within an rms value of .0005 volts into $50 \mathrm{ohms}$ would have an average power of $-53 \mathrm{dBm}$. 
Each digitizer contains a 512 Kword (1 MByte) buffer memory that can be partitioned for multiple recordings using software. The recorded data can then be transferred to the Radisys DOS-based drive or to a VXI drive or processor.

In 1997, a standard PC and a HP-based MXI interface replaced the Radisys controller to provide more flexibility, more data storage and a better user interface. A software interface to a VXI drive was also developed for faster HP Local Bus transfers. The advertised transfer rate of the buffer memory data to other devices was 2-4 MWord/second via the VME bus and 20-40 MWord/second via the HP Local bus. However, the observed transfer rates were significantly less. During September 1997, the observed HP local bus transfer rate from the digitizers to the VXI disk drive was on the order of $20 \mathrm{MBytes} / \mathrm{second}$ while the transfer of the data from the VXI drive to the DOS drive was on the order of $64 \mathrm{KBytes} / \mathrm{sec}$. The transfer rate from the digitizers to the DOS drive using the Rome Research software was in the order of $10 \mathrm{KBytes} / \mathrm{second}$.

In 1998, a LeMans VX8 DSP board was installed into the VXI system. This board currently contains two C40 modules and two dual C44 modules to provide up to 720 MFLOPS of peak processing power. This system and its associated software are described in Chapter 5 of this report. Delays in the delivery of some of the development hardware and software prevented to use of the DSP module in 1998. The data presented in this report was collected using the Rome Research software. 


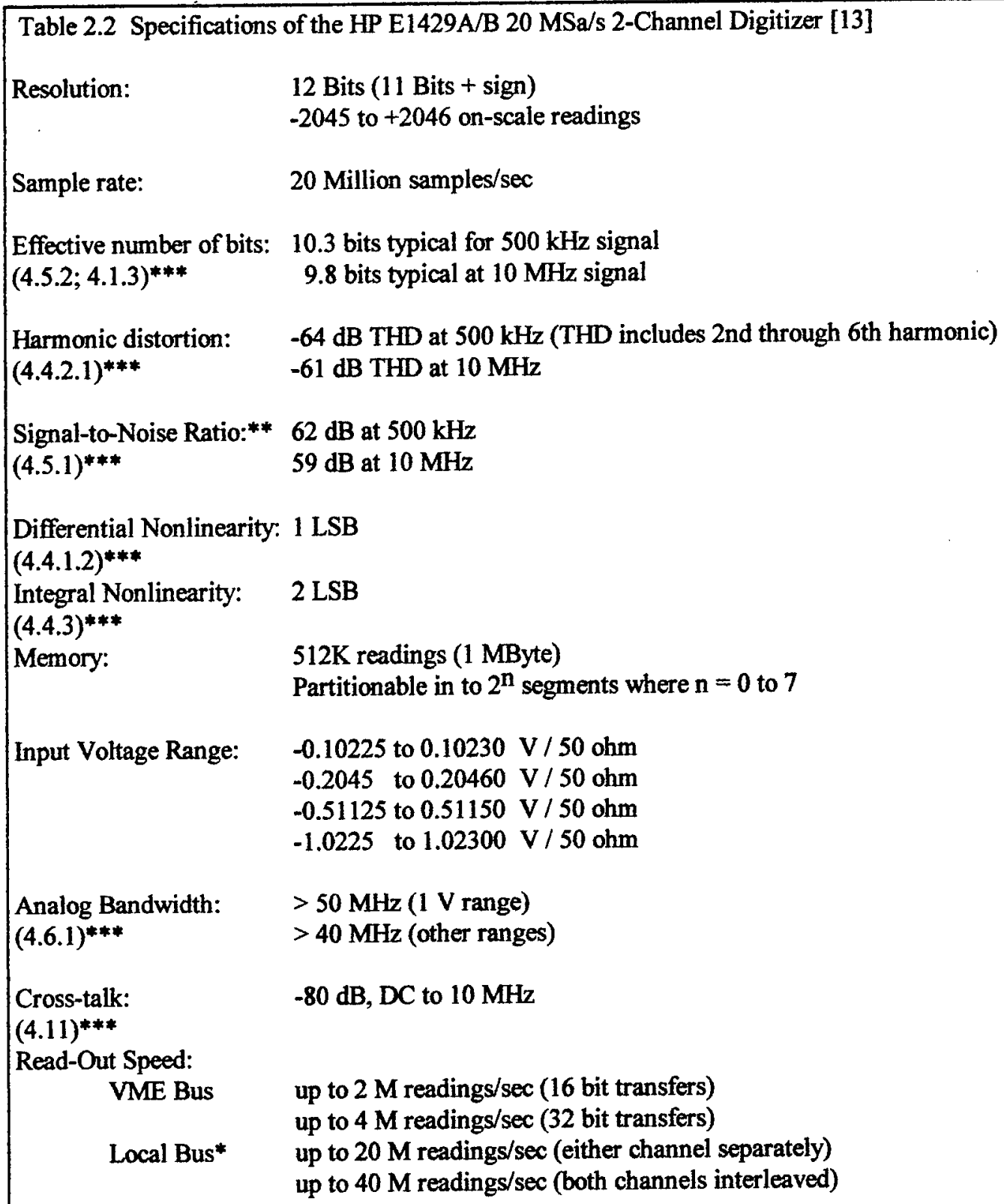

* readings may be routed to Local Bus during digitization and simultaneously with recording in the internal memory

** includes noise, distortion and all other undesired effects as defined in IEEE 1057

*** refers to sections in IEEE Standard 1057 


\subsection{ATTENUATION OF TREES AND FOLIAGE AT S-BAND FREQUENCIES}

The attenuation of trees and foliage has been of interest to many commercial and military applications for many years. Commercial interests include the impact of trees on communications systems and on the capability of remote sensing systems to provide environment information. An example of military interest is the detection and tracking of stationary and moving military targets in foliage.

Few measurements of the attenuation of trees and foliage have been performed at microwave frequencies. Ulaby, Whitt and Dobson [14] provides a discussion of the general problem and summarizes some of the difficulties associated with the propagation in a forest canopy. This paper presents the attenuation from a dense canopy of pine trees at $1.6 \mathrm{GHz}$. A polarimetric scatterometer was placed on a 19 meter platform and one-way measurements of attenuation were measured at a 40 degree incidence angle. A mean values of the one-way attenuation factors were reported as $9.31 \mathrm{~dB}$ for horizontal polarization and $9.16 \mathrm{~dB}$ for vertical polarization.

Multi-frequency measurements performed by MIT Lincoln Lab in 1990 were reported by Fleishman et al. [15]. These measurements were performed at UHF, L-Band and C-Band and used NASA/JPL's fully-polarimetric Synthetic Aperture Radar (SAR). The measurements were performed through a forest in Maine. The reported results included the median values and distributions of the two-way attenuation through the forest canopy as a function of frequency, polarization and depression angle. The authors concluded that the frequency dependence of the median attenuation is approximately $\mathrm{f}^{0.6}$ where the attenuation is expressed in $\mathrm{dB}$.

While the attenuation values increase as a function of frequency, the attenuation values are not excessive at the lower microwave and upper UHF frequencies. This is an important consideration for airborne foliage penetration systems where the detection of slowly moving

\begin{tabular}{|ccccccc|}
\hline \multicolumn{6}{|c|}{ Table 3.1 Median attenuation through a forest canopy in Maine [15] } \\
Depression & C-Band & L-Band & UHF \\
Angle & HH & VV & HH & VV & HH & VV \\
$($ deg.) & $(\mathrm{dB})$ & $(\mathrm{dB})$ & $(\mathrm{dB})$ & $(\mathrm{dB})$ & $(\mathrm{dB})$ & $(\mathrm{dB})$ \\
30 & 33.6 & 32.8 & 17 & 17.1 & 5.5 & 10.5 \\
45 & 30.4 & 27.5 & 12.7 & 13.3 & 5.4 & 9.5 \\
60 & 24.2 & 23.1 & 11.0 & 10.4 & 4.0 & 5.4 \\
\hline
\end{tabular}


targets uses coherent side-looking synthetic aperture processing and AMTI. Raney [16] shows that the negative effects of target motion are decreased as the radar scan rate increases and as the Doppler beam sharpening is decreased. Both of these system parameters favor higher frequency operation. The L-Band and S-Band frequencies represent a compromise between the canopy attenuation and the desirable system parameters identified by Raney.

The AFRL bistatic testbed at the Newport site provides the opportunity to perform S-band measurements of the local forest attenuation and the detection of moving targets screened by vegetation. The 1998 version of the testbed required 15 to 30 minutes to perform a measurement and download it to storage. This operation did not allow for the large number of frequent measurements needed to perform a statistical analysis of the attenuation issue. However, it was useful in determining if the S-Band attenuation was small enough to entertain a serious investigation into an S-Band foliage penetration system in the near future.

\subsection{Attenuation Measurements}

Three sites were chosen for the attenuation measurements. The town barn site used in previous experiments [6-9] was chosen as the reference site. It offered no screening from vegetation and, except for a few houses, provided a clear reference site. The past measurements at this site also provide a baseline for checking the radar system. A site on Newport Road provided a site with minor screening from tree canopies and small vegetation near the road. This site would provide small-to-moderate attenuation levels comparable to the attenuation provided by leaves and small branches to short range, high depression angle airborne platforms. The third site provides a variable degree of dense vegetation where attenuation is provided by tree trunks as well as leaves and smaller branches. This situation is more typical of a stand-off MTI system that uses Pulse Doppler techniques to find screened moving targets. The next three sections present the measurements at each of the three sites.

\subsubsection{Town Barn Site}

The site near the town barn was used in the past experiments because it provides an unscreened view to both the transmitter and receiver and provides natural screening to reduce the effects of multipath. Consistent results were reported in past reports and this site was used to provide a check on the operation of the radar system and to provide a reference for other site measurements. The site is also near a road that allows a reference measurement of the vehicle used in the other screened measurements [9]. 
Figure 3.1 presents the topographic display showing the location of the town barn location. The display of the range-angle radar data on the $\mathrm{x}$ $y$ coordinates requires a coordinate transformation. The isorange contours of a bistatic system are described by an ellipse where the foci are the transmitter and receiver locations [17].

$$
\frac{x^{2}}{a^{2}}+\frac{y^{2}}{b^{2}}=1
$$

where

$$
a_{i}^{2}-b_{i}^{2}=\frac{R_{b a s e l i n e}^{2}}{4}
$$

For a given $x-y$ coordinate on the map, the total time delay within the data window can be

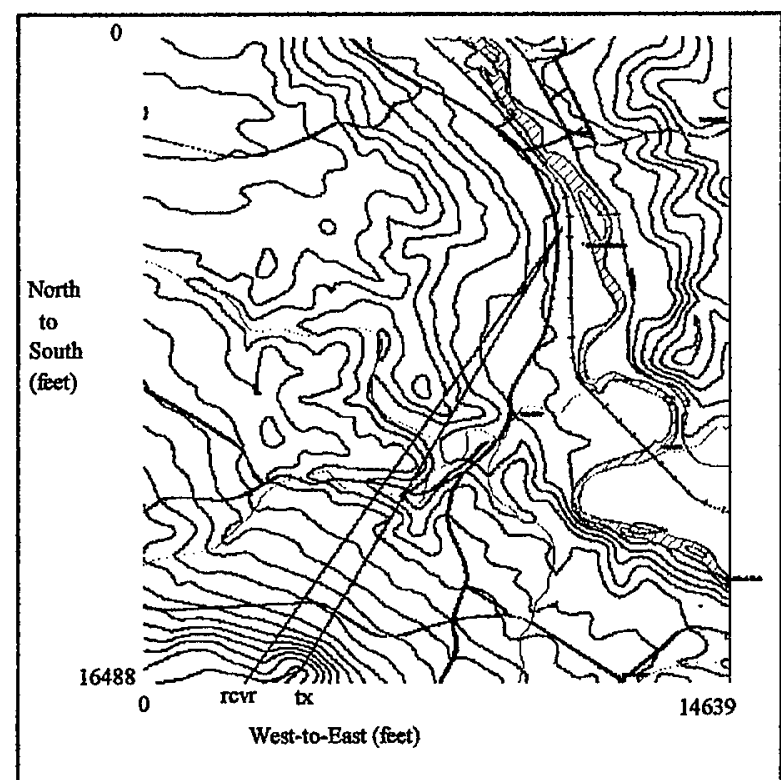

Figure 3.1 Topographic map of town barn reference site given as

$$
t=\frac{r_{r}+r_{t}}{c}-t_{L}
$$

where, from geometry,

$$
\begin{aligned}
& r_{r}=\sqrt{\left(x-x_{r}\right)^{2}+\left(y-y_{r}\right)^{2}} \\
& r_{t}=\sqrt{\left(x-x_{t}\right)^{2}+\left(y-y_{t}\right)^{2}}
\end{aligned}
$$

and $t_{L}$ is the time for the energy to transit the bistatic baseline. The angle for the $(x, y)$ point as measured at the receiver can be given as

$$
\text { angle }=\operatorname{atan}\left(-1^{*} \frac{\left(x-x_{r}\right)}{(y-y r)}\right)+\theta_{\text {scan }}
$$

where $\theta_{\text {Scan }}$ is the angle of the beam relative to true north and atan corresponds to the arc tangent.

The mapping from polar to rectangular coordinates is limited to within 90 degrees of the broadside azimuth. Areas corresponding to other angles are given as white or other default color. The propagation time for the signal to transit the baseline is $\sim 1.6$ microseconds. Most of the other 
Table 3.2 Town barn calibration measurements

$\begin{array}{cc}\mathrm{Tx} & \text { Rcvr } \\ \mathrm{dBm} & \mathrm{dBm} \\ 53.1 & 49.2 \\ 52.9 & 52 . .0\end{array}$

Range from transmitter: $12215 \mathrm{ft}$

Range from receiver: $13635 \mathrm{ft}$

Effective RCS of calibrator $=33.5 \mathrm{dBsm}$ at $250 \mathrm{~Hz}$ frequency offset.

Waveform: 40 usec pulse, $5 \mathrm{MHz}$ LFM, Hamming weight, $500 \mathrm{~Hz}$ PRF

Pulse Processing: 3 pulse MTI, 32 pulse DFT, Hanning weight

Antenna weighting: Taylor wt, $35 \mathrm{~dB}$ sidelobe, nbar $=6$

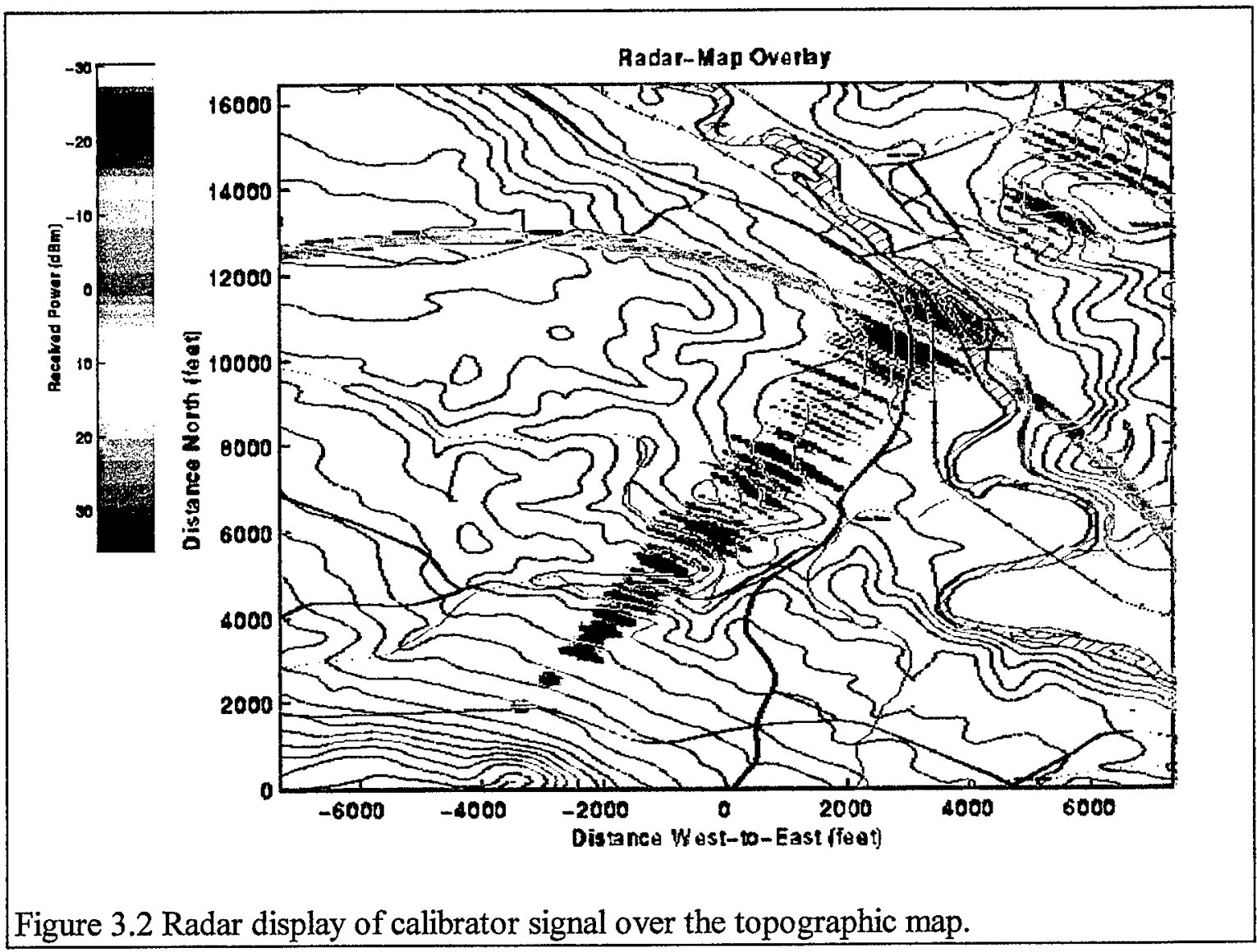


delays in the system consist of the delay in the fiber optic line between the receiver shelter and the transmitter and the delays in the up-converter and down-converter .

Figure 3.2 shows the radar display superimposed on the terrain map shown in Figure 3.1. The strong return from the calibrator is located near the road passing the Newport town barn. The elliptical band caused by the mainbeam multipath discussed in [9] is evident. The band appears more circular because the terrain map dimensions were distorted to fit the rectangular format of the radar display. The weak range sidelobe responses are also evident.

Table 3.2 presents the calibration data taken during the 1998 tests. The major differences from the 1997 data [9] are the $13.4 \mathrm{~dB}$ increase in gain and effective RCS of the calibrator, the use of a 3 pulse MTI in the processing and the number of pulses coherently integrated. The interfering ground clutter return from this area averages $11 \mathrm{dBsm}$ with peak returns up to 20 $\mathrm{dBsm}$. After MTI and DFT processing, this interference is over $60 \mathrm{~dB}$ below the calibration signal $[8,9]$. 


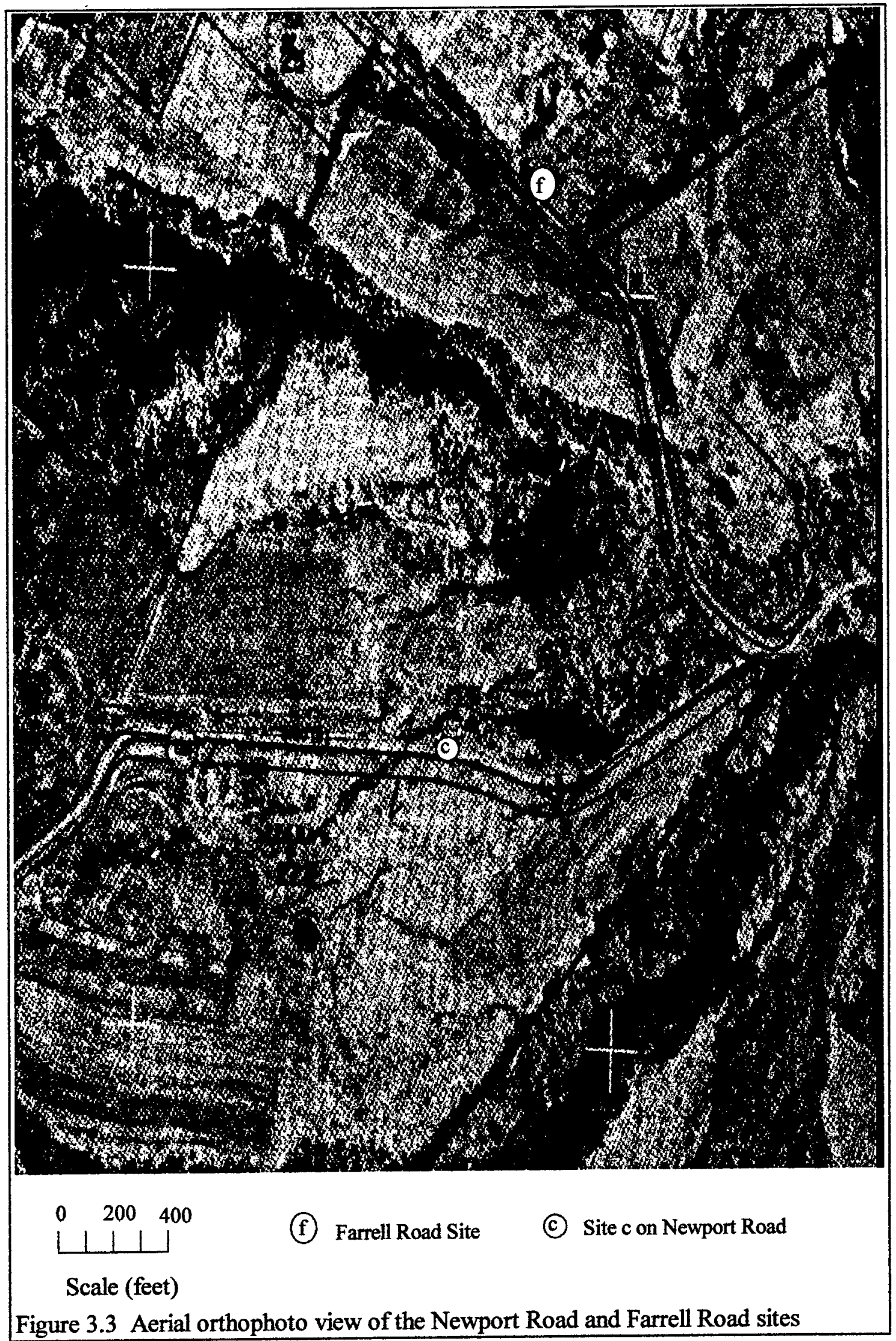




\subsubsection{Screened Measurement Sites}

Figure 3.3 presents an orthophoto map of the region around the measurement sites on Newport Road and Farrell Road. [18]. The $c$ and $f$ letters show the location of the calibration reference sites on Newport Road and Farrell Road, respectively, that provided a non-screened view of both the transmitter and the receiver. The nine sites along Farrell Road provided varying degrees of screening by a mixture of leaves, branches and tree trunks near the road as well as canopies from tall trees up to 500 feet from the road. The Newport Road site was chosen because it provided optical screening by the upper canopy of one or two fully leafed maple and birch trees as well as by small trees and weeds near the road. The map in Figure 3.3 was taken in Oct. 30, 1978 and does not show the small trees near road or the larger trees in the pasture within 500 feet of the road. Figure 3.4 presents a recent view toward the Newport and Farrell Road sites from the receiver. The $\mathrm{c}$ and $\mathrm{f}$ indicate the calibrator reference sites that were unscreened to both the transmitter and the receiver.

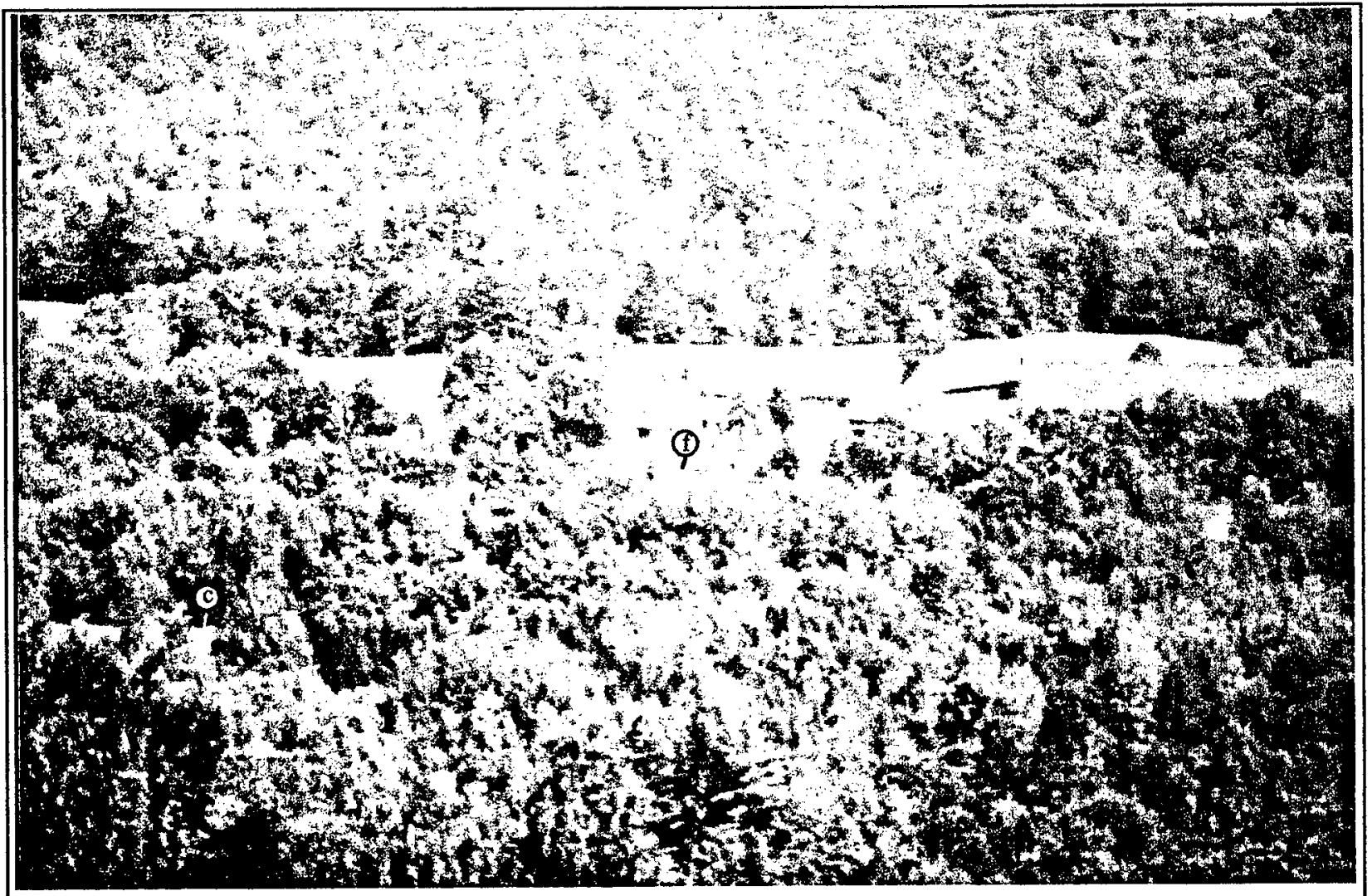

Figure 3.4 View of Newport Road and Farrell Road reference sites from the bistatic receiver. 


\subsubsection{Measurements at the Farrell Road Site}

The reference site is shown as site $f$ in Figures 3.3 and 3.4. The other eight measurement sites exist at 50 foot intervals west (to the left) of this reference point with various degrees of screening by the trees and vegetation near the rod. Descriptions and one-way attenuation were performed at only the 100 foot intervals. Additional attenuation was provided along the receiver path by the tops of a second row of trees about 500 feet from the road. The width of this interfering canopy is no more than one tree canopy width and varies from $\sim 10$ feet to $\sim 50$ feet. This second row of trees provides no attenuation to the transmitter signal for any of the nine sites along this road. However, the tops of the trees are within both the transmitter's and receiver's antenna beamwidth and probably contribute multipath components that can provide small ripple in the energy field.

Figure 3.5 shows the unscreened view of both the transmitter and the receiver from the reference site. The transmitter ray path is well above the trees while the path to the receiver is between the tops of two trees located about 500 from the road. The brush near the road helps to

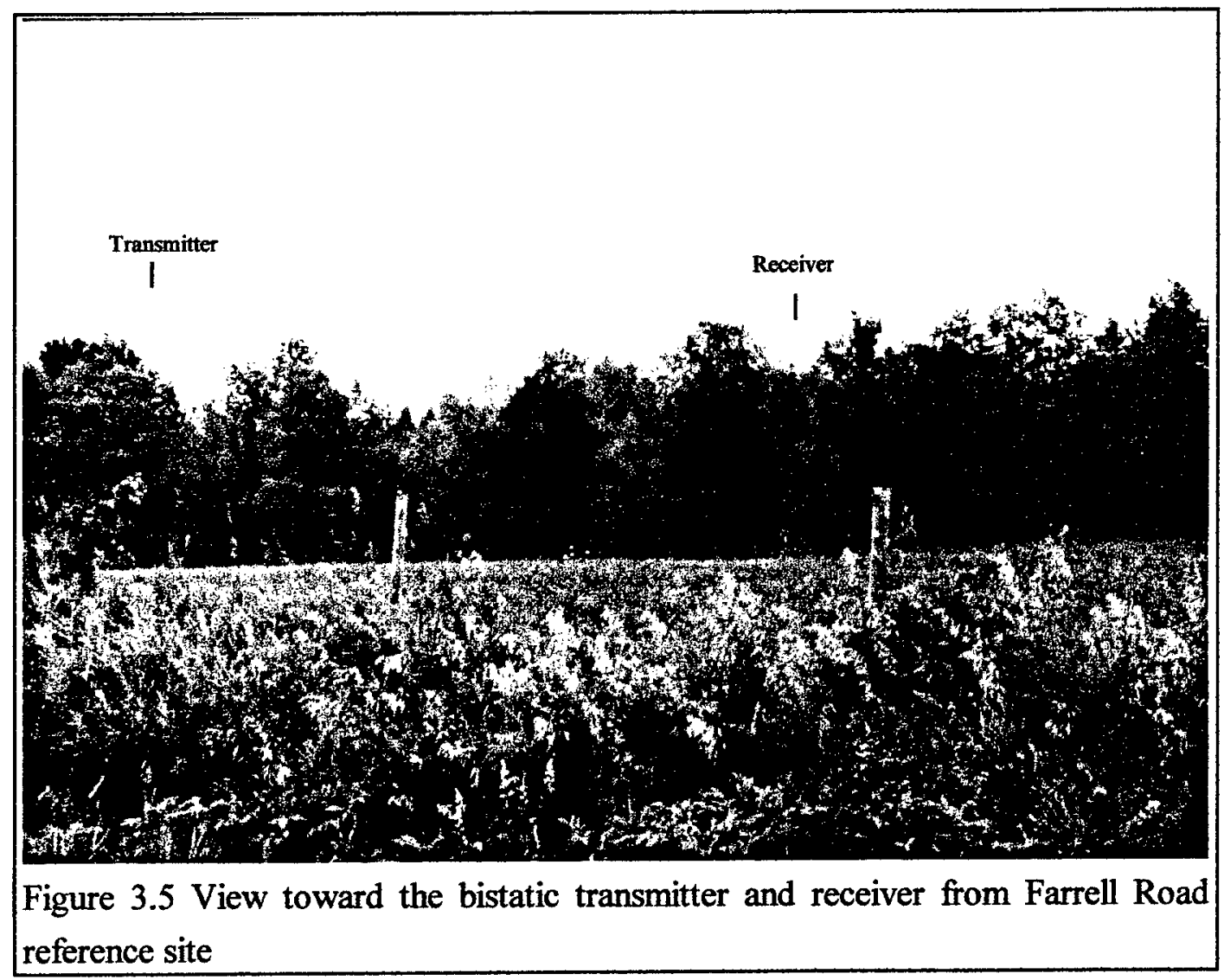


reduce the specular multipath components.

Figure 3.6 shows the small sugar maple trees screening the measurement site at 100 feet West of the reference point. This screening is present for both the transmitter and receiver. The maximum width of the tree's foliage along the ray paths was measured at 9 feet. A barb wire fence with 2 strands, at two and four feet above the ground, is also within these small trees. Additional screening in receiver direction by upper canopy of one tree located about 500 feet from the road. The canopy is estimated to be 10 feet deep.

Figure 3.7 shows a denser screening in both the transmitter and receiver directions at 200 feet West. The screening is provided by small and medium size trees, mostly sugar maple and birch. The width of the tree's foliage along the ray paths was measured at 17 feet. A barb wire fence with 2 strands, at two and four feet above the ground, is also within these trees. Additional screening in receiver direction was provided by upper canopy of one tree about 500 feet from the road. The estimated width of this interfering canopy is 25 feet. A dip in road placed the road surface below grade, causing additional screening by the ground of scatterers near the road surface.

At 300 feet West, denser screening exists in both transmitter and receiver direction by small and medium trees near the road, mostly sugar maple and birch. The width of the tree's foliage along the ray paths is about 55 feet with sparse foliage for the 22 feet near the road and heavy foliage and trunks for the next 33 feet. A barb wire fence with 2 strands, at two and four feet above the ground, is also within these trees. Road is rising from the dip at 200 feet and is $\sim 1$ foot below sides.

Figure 3.8 shows the densest screening at this site. Screening in both transmitter and receiver direction is provided by small and medium trees, mostly sugar maple and birch. The width of the tree's foliage along the ray paths was measured at 118 feet. A barb wire fence with 2 strands, at two and four feet above the ground, is also within these trees. Additional screening in receiver direction was provided by upper canopy of one large tree about 500 feet from the road. The width of the interfering part of this canopy is about 50 feet deep. Road is even with sides.

One-way measurements were performed using one of the calibrator horns and a spectrum analyzer calibrated for use with an uncoded 40 microsecond waveform [9]. Compass headings were used to align the horn with the transmitter because the screening made visual alignment impossible. Table 3.3 summarizes the results when the horn was aligned with the transmitter. The depth of the RF shadow ranged from $8 \mathrm{~dB}$ for a single row of young maple trees to $17 \mathrm{~dB}$ in an 

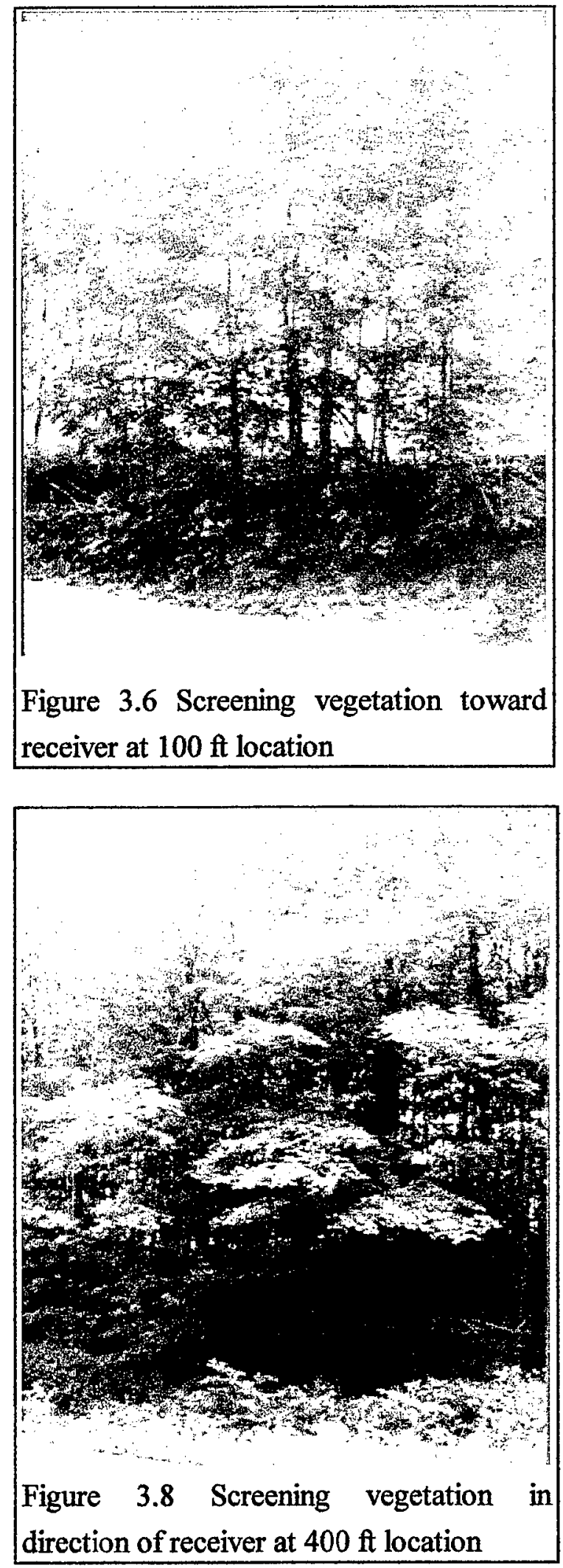
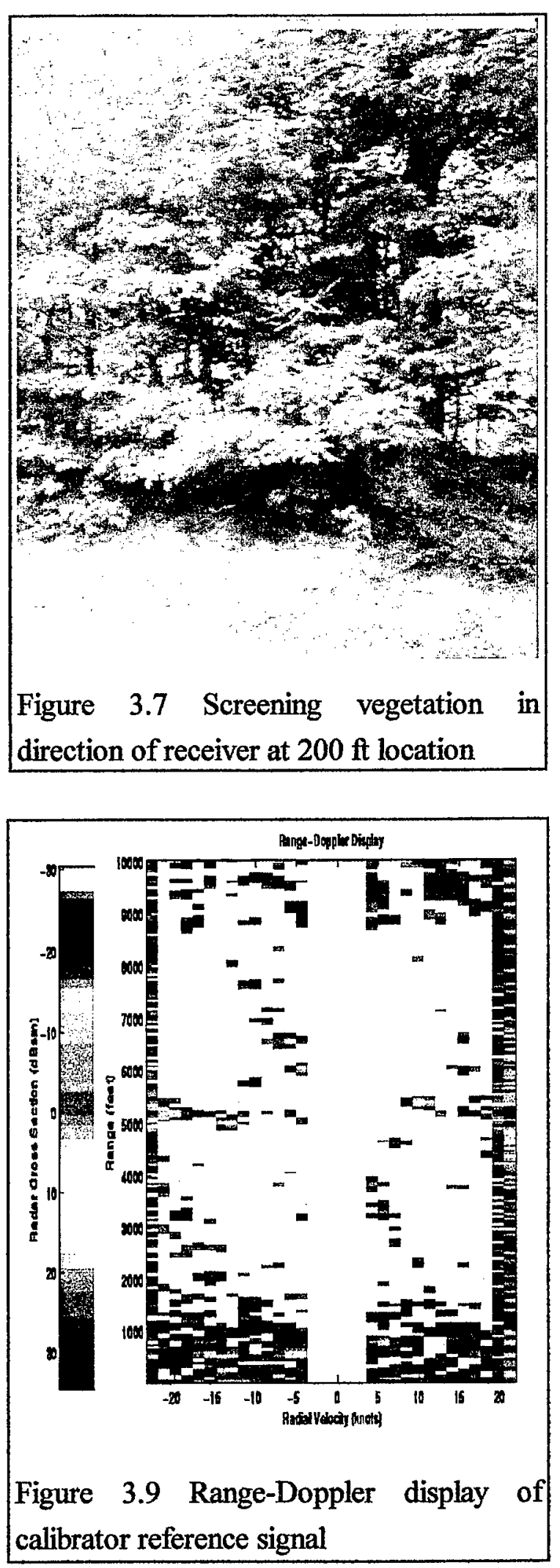
areas with significant screening. Within the heavily screened areas, significant multipath was noted from directions other than the transmitter direction. Notably, the multipath signal down the road in the direction of the reference site was nearly as strong as the direct transmitter signal. This signal is probably bistatic scatter from the small trees noted near the reference site and from larger trees that were on the other side of the road.

Bistatic measurements made use of the calibrator. In the screened areas, both the receive and transmit horns were aligned using compass headings. The clutter from the area ranged between 15 and $20 \mathrm{dBsm}$. The MTI and DFT processing attenuated the clutter to the equivalent of less than $-30 \mathrm{dBsm}$ while providing 5 to $26 \mathrm{~dB}$ of integration gain to the calibration signal. This provided up to by the 3PMTI and the DFT processing. This provided up to $65 \mathrm{~dB}$ of useful dynamic range for the measurement of foliage attenuation. Figure 3.9 presents the range-Doppler display of the calibrator reference demonstrating this dynamic range.

Table 3.4 summarizes the bistatic attenuation measurements taken at the Farrell Road site. Figure 3.10 presents this data as a function of location while Figure 3.11 presents this data as a function of foliage screening depth. One impression given by the measurements is that moisture impacts the attenuation at least for the shallow depths. The July 1 measurements were taken between heavy rain showers while the 13 July measurements were taken after several hot dry days had thoroughly dried the leaves. Second, for the first 10 or 20 feet of intervening foliage, the rates of one-way attenuation versus depth were approximately $1 \mathrm{~dB} /$ foot for dry conditions and 2 $\mathrm{dB} /$ foot for wet conditions. However, beyond 20 feet of foliage depth, the measured energy ceased to decrease indicating that the attenuated direct signal was not the only illuminating energy source. It is suggested that multipath scatter from the nearby trees, leaves and structures becomes the dominant illuminating energy as the direct signal becomes severely attenuated. 
Table 3.3 One way attenuation measurements at the Farrell Road site

Site

$\begin{array}{ccccc}\mathrm{Tx}^{*} & \mathrm{~T}_{\mathrm{cal}}{ }^{* *} & \text { Pcal }^{1} & \text { One-way }^{2} & \text { Screening } \\ \mathrm{Az} & \mathrm{Az} & \text { meas. } & \text { Path Loss } & \text { Depth } \\ (\mathrm{deg}) & (\mathrm{deg}) & (\mathrm{dBm}) & (\mathrm{dB}) & (\mathrm{ft})\end{array}$

Date: $08 / 05 / 98$

$\begin{array}{lccccc}\text { Reference } & 286.3 & 176 & -2 & 0 & -- \\ 100 \mathrm{~W} & 286.3 & 175 & -12 & -8 & 9 \\ 200 \mathrm{~W} & 286.3 & 174 & -17 & -15 & 17^{3} \\ 300 \mathrm{~W} & 286.5 & 173 & -15 & -13 & 33^{4} \\ 400 \mathrm{~W} & 286.5 & 172 & -19 & -17 & 118\end{array}$

* azimuth angles are local measurements logged for reference

** Calibration alignment relative to magnetic north

$\mathrm{Tx}=52.5 \mathrm{dBm}, \mathrm{Tx} . \mathrm{El}=-5.6 \mathrm{deg}$.

Waveform: 40 usec pulse, uncoded

${ }^{1}$ The reference value is close to the theoretical free-space value using the estimates given in [9]

2 The measured power within the screened areas exhibited a noisy lobing structure with nulls

typically 8 to $10 \mathrm{~dB}$ less than the peak. The calibration horn was set at a height that was within 3 $\mathrm{dB}$ of the maximum signal.

3 Some attenuation is likely due to the dip in the road which placed the calibrator height at the height of the shoulder.

422 feet depth of sparse short brush was also present near the road. 
Table 3.4 Bistatic attenuation measurements at Farrell Road site

$\begin{array}{lcccccc}\text { Site } & \mathrm{Tx}^{*} & \mathrm{~T}_{\mathrm{cal}}{ }^{* *} & \mathrm{R}_{\mathrm{cal}}^{* *} & \text { Bistatic } & \text { Bistatic } & \text { Screening } \\ & \mathrm{Az} & \mathrm{Az} & \mathrm{Az} & \text { Signal } & \text { Attenuation } & \text { Depth } \\ & (\mathrm{deg}) & (\mathrm{deg}) & (\mathrm{deg}) & (\mathrm{dBm} / \# \mathrm{p}) & (\mathrm{dB}) & (\mathrm{ft})\end{array}$

Date: $06 / 24 / 98$

Reference $\quad 286.0 \quad 176 \quad 191+53.2 / 16$

Date: $07 / 01 / 98^{2}$

$\begin{array}{lcccccr}\text { Reference } & 286.1 & 176 & 191 & +65.2 / 128 & 0 & --- \\ 50 \mathrm{~W} & 286.1 & 176 & 191 & +47.7 / 128 & 17.5 & 9 \\ 100 \mathrm{~W} & 286.1 & 175 & 190 & +44.4 / 128 & 20.8 & 9 \\ 200 \mathrm{~W} & 286.1 & 174 & 189 & +17.4 / 128 & 47.8 & 17 \\ 300 \mathrm{~W} & 286.1 & 173 & 188 & +23.0 / 128 & 42.2 & 33 \\ 400 \mathrm{~W} & 286.1 & 172 & 187 & +22.8 / 128 & 42.4 & 118\end{array}$

Date: $07 / 13 / 98^{3}$

$\begin{array}{lcccccc}\text { Reference } & 286.1 & 176 & 191 & +48.6 / 16 & 0 & --- \\ 50 \mathrm{~W} & 286.1 & 176 & 191 & +38.4 / 16 & 10.2 & 9 \\ 150 \mathrm{~W} & 286.1 & 175 & 190 & +30.7 / 16 & 17.9 & 16 \\ 250 \mathrm{~W} & 286.1 & 173 & 188 & -6.5 / 16 & 55.1 & 33 \\ 350 \mathrm{~W} & 286.1 & 172 & 187 & +4.5 / 16 & 43.1 & 115\end{array}$

* azimuth angles are local measurements logged for reference

** Calibration alignment relative to magnetic north

$\mathrm{Tx}=52.5 \mathrm{dBm}, \mathrm{Tx} . \mathrm{El}=-5.6 \mathrm{deg}$.

Range from transmitter: 6225 feet

Range from receiver: 6370 feet

effective RCS of calibrator $=33.5 \mathrm{dBsm}$ at $250 \mathrm{~Hz}$. modulation

Waveform: 40 usec pulse, $5 \mathrm{MHz}$ LFM, Hamming weight, $500 \mathrm{~Hz}$ PRF

Antenna weight: Taylor $w t, 35 \mathrm{~dB}$ sidelobe, $\mathrm{nbar}=6$

2 wet leaves (July 1, 1998)

Pulse Processing: 3 pulse MTI, 126 pulse DFT, Hanning weight

3 dry leaves (July 13, 1998)

Pulse Processing: 3 pulse MTI, 14 pulse DFT, Hanning weight 


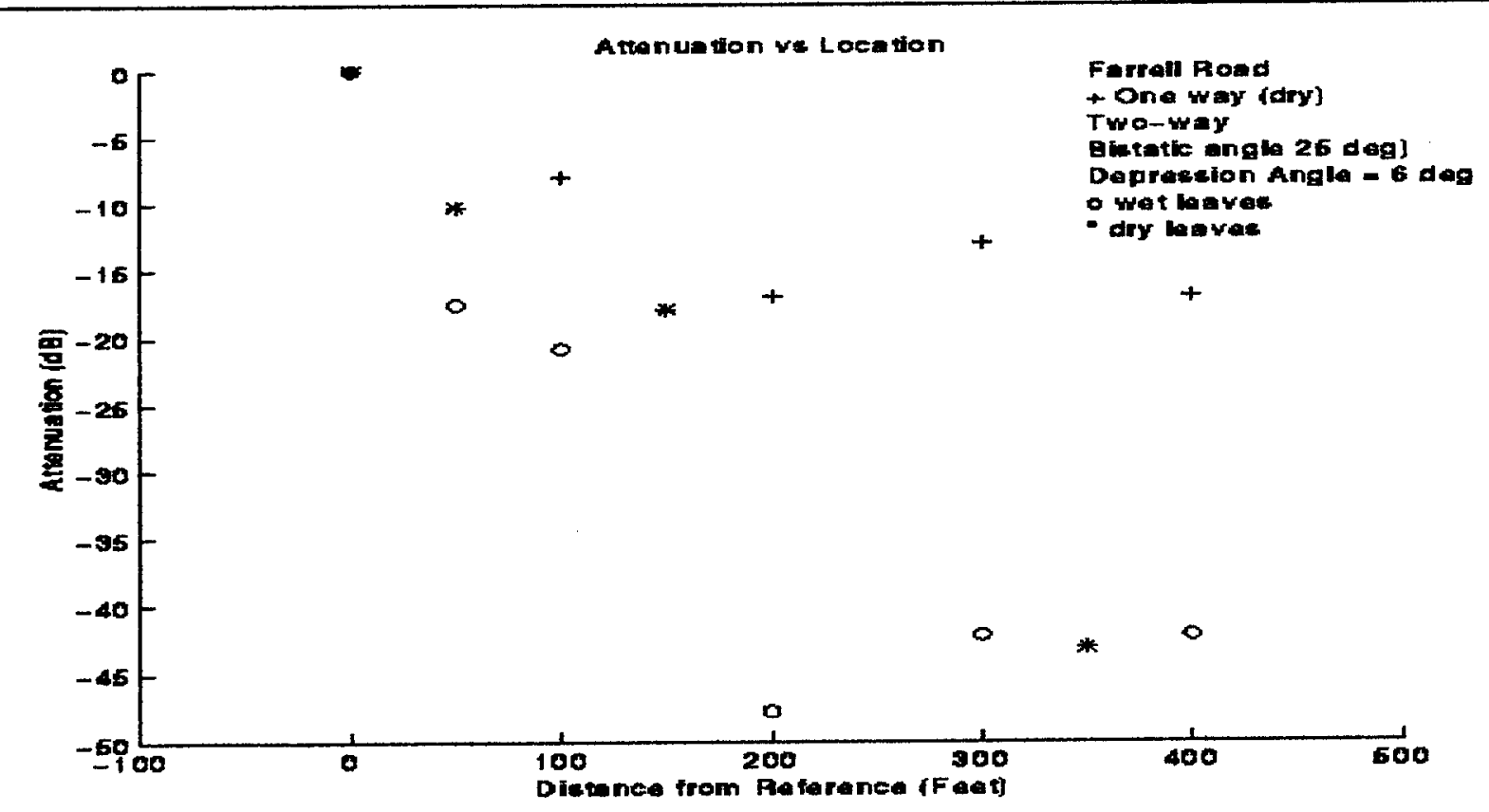

Figure 3.10 Foilage attenuation measurements as a function of location

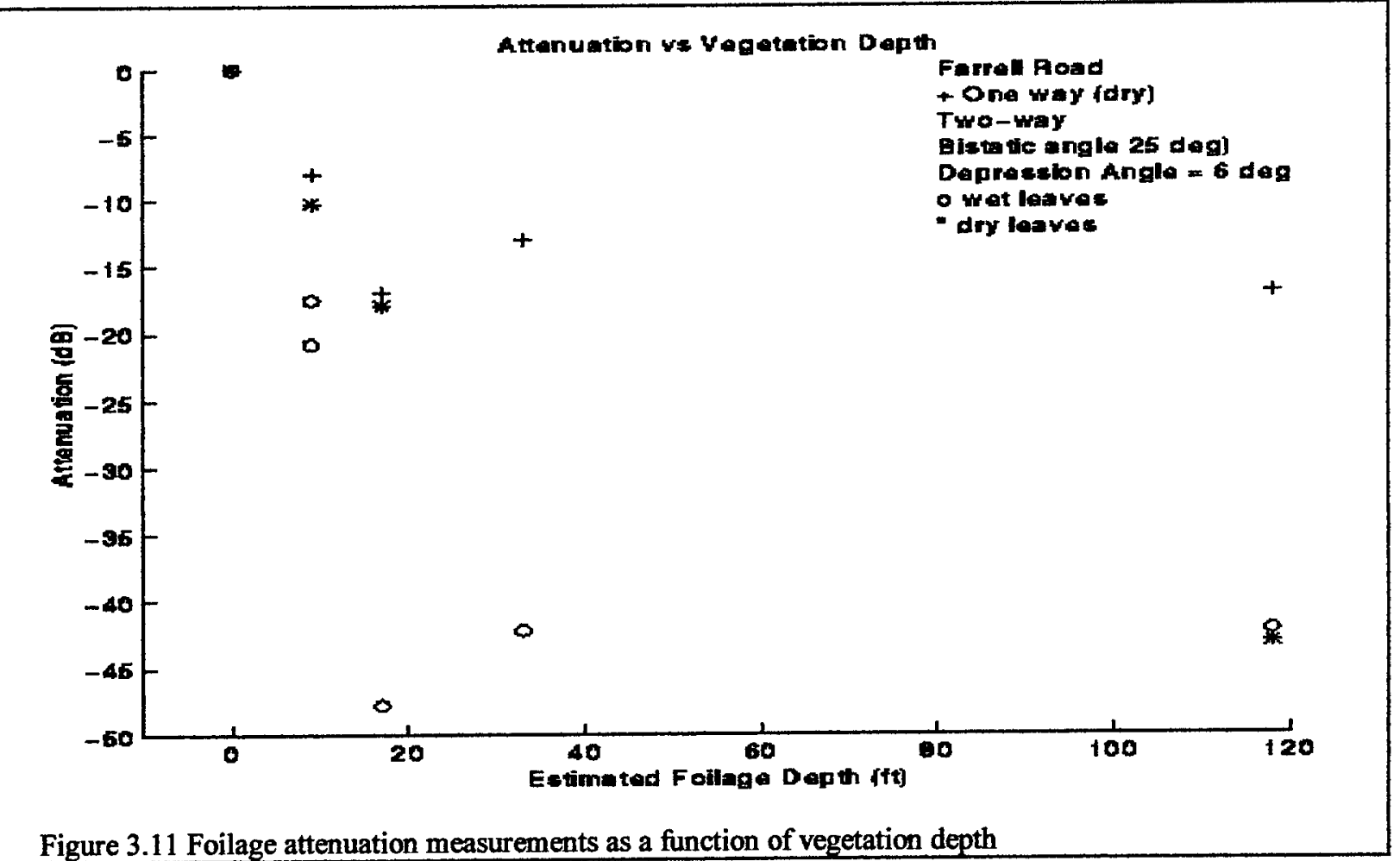




\subsubsection{Measurements at the Newport Road Site}

The Newport Road site was chosen because it provided optical screening by the upper canopy of one or two fully leafed maple and birch trees as well as by small trees and weeds near the road. At the test locations on either side of reference site, the calibrator and vehicles are optically screened from both the transmitter and receiver by foliage from small trees and brush near the road. The light-to-moderate attenuation by this screening is comparable to that provided to airborne sensors at higher depression angles. The canopy of taller trees about 800 hundred feet from the road are below the ray path but within the receiver main beam. Tree canopies below and to each side of the ray path are also within main beam and provide multipath scatter. The site also provides a near tangential path for tests of low-RCS low-radial velocity measurements.

Five points near this site were selected for attenuation measurements. Canopy widths and the distances of the trees from the road were estimated because of the limited access to the site. The first site was the reference site. Brush and weeds were sparse within 50 feet of the road along each radial, creating the possibility for multipath lobing.

The second point was 100 feet west (to the left in the figure). This location had a sparse row of small trees in direction of both transmitter and receiver. The receive path received additional attenuation from a canopy of one tree about 300 feet from the road.

The third point was 200 feet west of the reference location. The road at this point was slightly below grade such that scatterers within a foot of the road surface would be screened by the ground. One 3 foot deep row of sparse brush screened the calibrator from the transmitter and the receiver. When the transmitter is pointed at the reference location, this point receives about 5 $\mathrm{dB}$ less than the full transmit antenna gain.

The fourth point was 100 feet east of the reference site. Along both the transmitter and receiver paths, screening was provided by dense brush and 3 small maple trees within 30 feet of the road. The canopy of a large tree about 500 feet from the road provides further screening toward the transmitter. The leaves and branches near the top of another medium sized tree provides additional screening in the receiver direction.

The fifth point is located 200 feet east of the reference site. The location is optically screened from transmitter and receiver by foliage and branches from dense brush and several small trees within 30 feet of the road. Canopy of 3 large trees about 600 feet from the road provides further screening toward the transmitter. Canopy from 2 medium sized trees provides screening 
from the receiver direction. When the transmitter is pointed at the reference location, this point receives about $5 \mathrm{~dB}$ less than the full transmit antenna gain.

At sites where the transmitter and receiver were visible, the calibrator horns were set up and aligned by eye [9]. Compass readings were recorded for repeatability. At sites where the transmitter and/or the receiver could not be seen, the calibrator horns were aligned using compass readings. The radar cross section of the clutter in this region ranges from about $0 \mathrm{dBsm}$ on the west side to $15 \mathrm{dBsm}$ from the trees on the east side.

Table 3.5 presents the measurements performed at the Newport road.

\begin{tabular}{|c|c|c|c|c|c|c|}
\hline & $\begin{array}{c}\mathrm{Tx}^{*} \\
\mathrm{Az} \\
(\mathrm{deg}) \\
\end{array}$ & $\begin{array}{c}\mathrm{T}_{\mathrm{X}} \\
\mathrm{Tx} \text { Power } \\
(\mathrm{dBm}) \\
\end{array}$ & $\begin{array}{c}\operatorname{Rcc}^{*} \\
\mathrm{Az} \\
(\mathrm{deg}) \\
\end{array}$ & $\begin{array}{l}\text { Rec } \\
\text { Elev } \\
\text { (deg) } \\
\end{array}$ & $\begin{array}{l}P_{\text {cal }} \\
\text { meas. } \\
(\mathrm{dBm})\end{array}$ & $\begin{array}{l}\text { Bistatic } \\
\text { Attenuation } \\
\text { (db) }\end{array}$ \\
\hline \multicolumn{7}{|l|}{$\underline{070998}$} \\
\hline $200 \mathrm{ft} \mathrm{W}$ & 294.8 & 52.9 & 30.0 & -7 & 53.7 & 5.4 \\
\hline $100 \mathrm{ft} \mathrm{W}$ & 294.8 & 52.9 & 30.7 & -7 & 50.3 & 8.8 \\
\hline reference & 294.8 & 52.9 & 31.4 & -7 & 59.1 & 0 \\
\hline $100 \mathrm{ft} \mathrm{E}$ & 294.8 & 52.9 & 32.1 & -7 & 51.6 & 7.5 \\
\hline $200 \mathrm{ft} \mathrm{E}$ & 294.8 & 52.9 & 32.8 & -7 & 17.0 & 42.1 \\
\hline \multicolumn{7}{|l|}{$\underline{081498}$} \\
\hline 081998 & 294.8 & 53.1 & 31.4 & -7 & 54.9 & 0 \\
\hline reference & 294.8 & 52.9 & 31.4 & -7 & 55.4 & 0 \\
\hline $\begin{array}{l}* \text { azimuth } \\
\text { Range fror } \\
\text { Transmitte } \\
\text { Effective } \mathrm{I} \\
\text { Waveform } \\
\text { Pulse Proc } \\
\text { Antenna } \mathrm{H}\end{array}$ & $\begin{array}{l}\text { les are lo } \\
\text { ansmitte } \\
\text { levation } \\
\text { of calib } \\
\text { usec pu } \\
\text { ing: } 3 \text { pu } \\
\text { ht: Tavlo }\end{array}$ & $\begin{array}{l}\text { ocal measu } \\
\text { er: } 5030 \text { fee } \\
\text { angle: }-6.5 \\
\text { orator }=33 \text {. } \\
\text { ulse, } 5 \mathrm{MH} \\
\text { ulse MTI, } 1 \\
\text { or wt, } 35 \mathrm{~d}\end{array}$ & $\begin{array}{l}\text { urement } \\
\text { et; } \quad \text { F } \\
5 \text { degre } \\
.5 \mathrm{dBsr} \\
\text { Iz LFM } \\
126 \text { pul } \\
\text { 1B sidel }\end{array}$ & $\begin{array}{l}\text { s logged } \\
\text { Range fr } \\
\text { es } \\
\mathrm{n} \text { at } 250 \\
\text { Hamm } \\
\text { se DFT, } \\
\text { obe nbe }\end{array}$ & $\begin{array}{l}1 \text { for refe } \\
\text { om recei } \\
\text { Hz. } \\
\text { ing weig } \\
\text { Hanning } \\
\text { ar=6 }\end{array}$ & $\begin{array}{l}\text { verence } \\
\text { iver: } 4875 \text { feet } \\
\text { ht, } 500 \mathrm{~Hz} \text { PRF } \\
\text { g weight }\end{array}$ \\
\hline
\end{tabular}




\subsection{MOVING TARGET EXPERIMENTS}

Chapter 3 presented measurements of the propagation attenuation through foliage at various locations in the test sites. This chapter presents measurement of the effective radar cross section of a small moving vehicle passing through the same areas. Snapshot measurements were performed to measure the effective RCS and Doppler of a screened and unscreened vehicle.

The next section describes the test setup and the procedures used in the measurement. Chapter 4.2 presents unscreened measurements performed near the calibration site near the town barn. Chapter 4.3 presents the screened and unscreened measurements at the Newport Road site. Chapter 4.4 presents the screened and unscreened measurements at the Farrell Road.

\subsection{Test Setup and Procedures}

The range between the bistatic receiver and the test area ranged from 4875 feet to over 11000 feet, too far for the radar operator to visually determine when the vehicle entered the test areas. Also the vehicle was often optically screened from the operator. Therefore, the following procedure was used to assure that the vehicle was near the measurement point when the recording was performed.

The vehicle operator maintained a true speed of 30 mile per hour ( 26 knots). This speed was chosen to provide radial velocities at the target sites between 9 and 22 knots. At $30 \mathrm{mph}$, the vehicle is going at 44 feet per second. Markers were set at 100 foot intervals from the measurement sites. Two UHF transceivers were used to coordinate and perform the tests. When the vehicle reached $30 \mathrm{mph}$ and passed a prearranged marker, the driver signaled the radar operator via the UHF transceivers. The radar operator then delayed initiating the recording for the number of seconds needed for the vehicle to reach the test site.

\subsection{Vehicle Measurements at the Town Barn Site}

The town barn provides an unscreened reference point to confirm system operation. Figure 4.1 shows the geometry of the measurement site on the road near the town barn. Most of the road along this site is unscreened. The road direction is 33 degrees relative to the bistatic bisector. Therefore, vehicles travelling on the road present a radial velocity that is $83.9 \%$ of their true velocity. The radial component is $25 \mathrm{mph}$ or 21.8 knots for the test vehicle traveling at $30 \mathrm{mph}$. 
Table 4.1 presents the measurements taken on August 14, 1998. A 34 pulse burst was used and the Doppler processing consisted of a 3 pulse MTI canceller using binomial weights followed by a 32 pulse DFT using Hanning weighting. The test waveform was a 40 microsecond modulated with a $5 \mathrm{MHz}$. Linear Frequency (LFM) waveform. The range weighting used in the matched filter is the Hamming weighting which provides a nominal $40 \mathrm{~dB}$ sidelobe level.

Figure 4.2 presents the processed return from the 17th Doppler filter. The peak signal from the vehicle was in the 17th filter which has

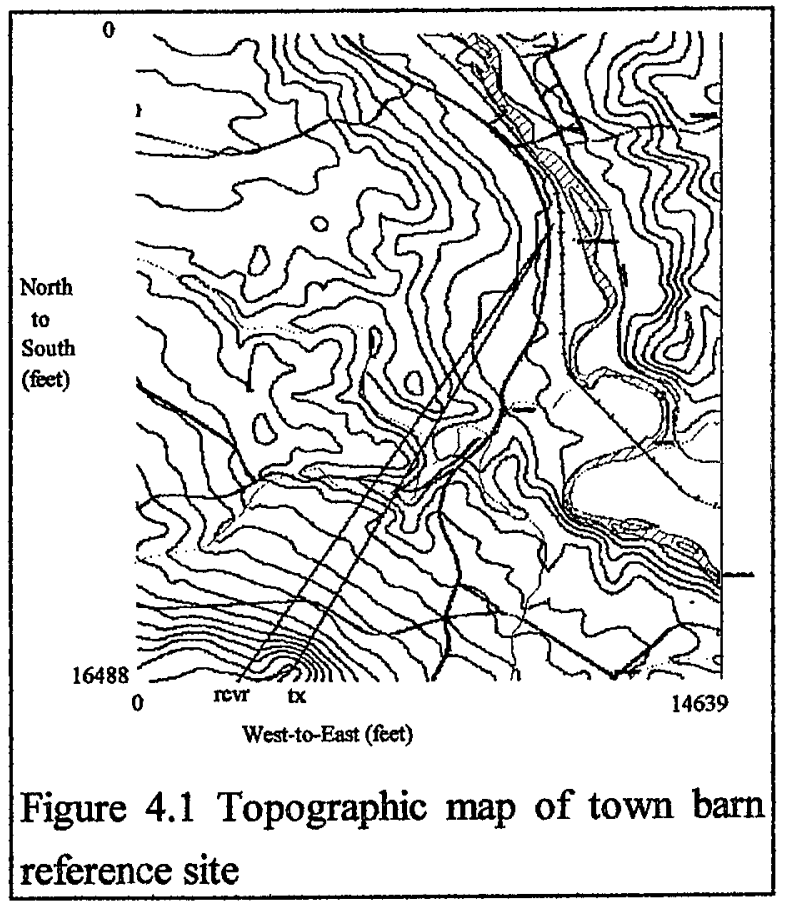
corresponds to a peak velocity of 21.0 knots for targets traveling toward the radar baseline or -23.8 knots for targets traveling away from the radar baseline. Figure 4.3 shows the effective radar cross section of the returns in the Auto 01 file versus range and radial velocity. The conversion to effective RCS was performed using a calibration constant derived using the calibrator, compensation versus range and a compensation filter for the 3 pulse MTI response. All of the velocity filters except the $5 \%$ centered at the zero velocity filter was multiplied by

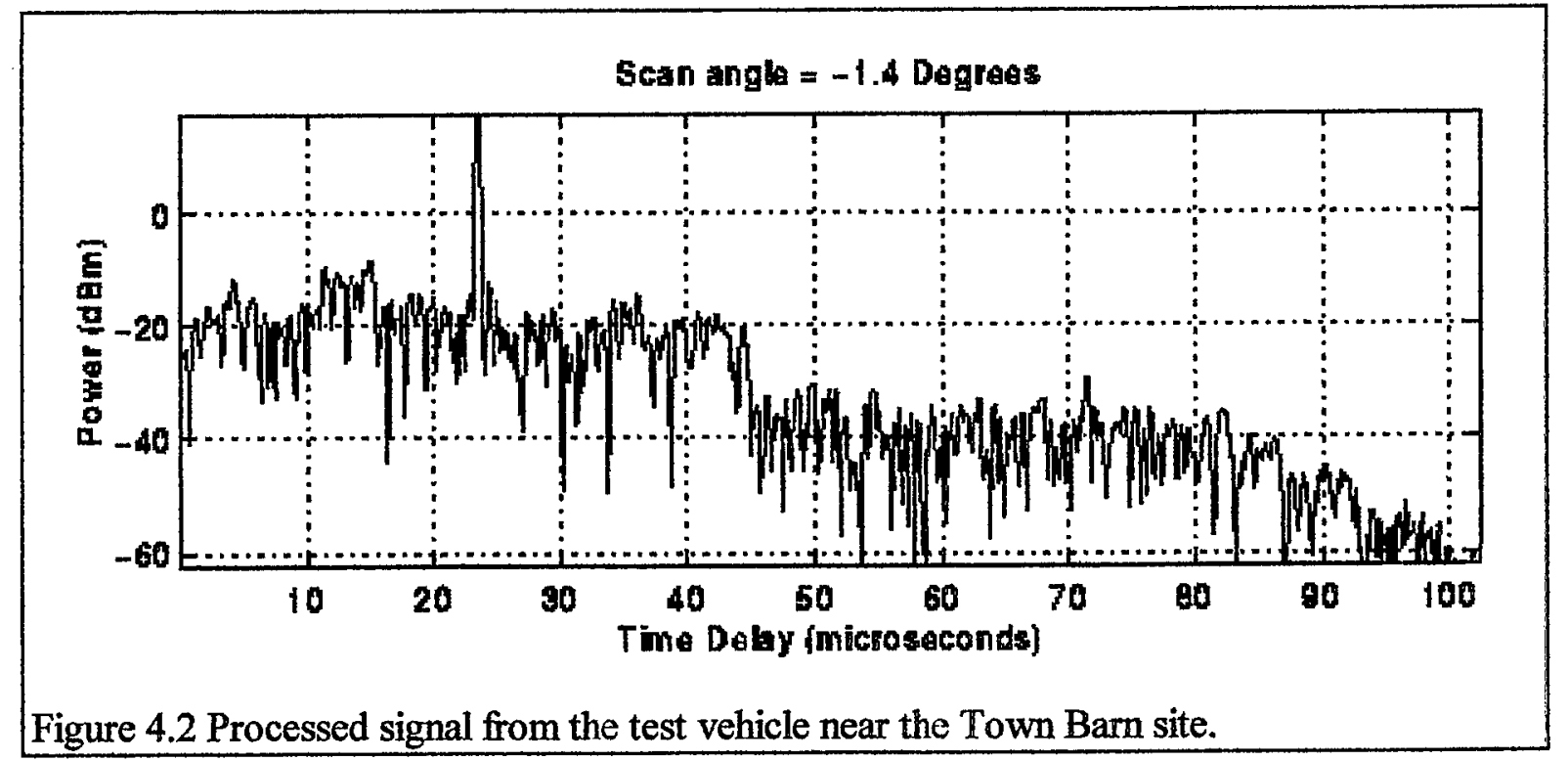




$$
H(f)=\left(\sin \left(\frac{f}{f_{\text {prf }}}\right)\right)^{-2}
$$

to compensate for the MTI filter. This display is used throughout this chapter to allow the reader to see the direct and multipath signals that are inherent in the screening problem. Given the simple compensation used, the inaccuracies of the RCS estimates are small near the location of the measurement sites, but increase at other ranges in the display.

The auto return is the relatively strong

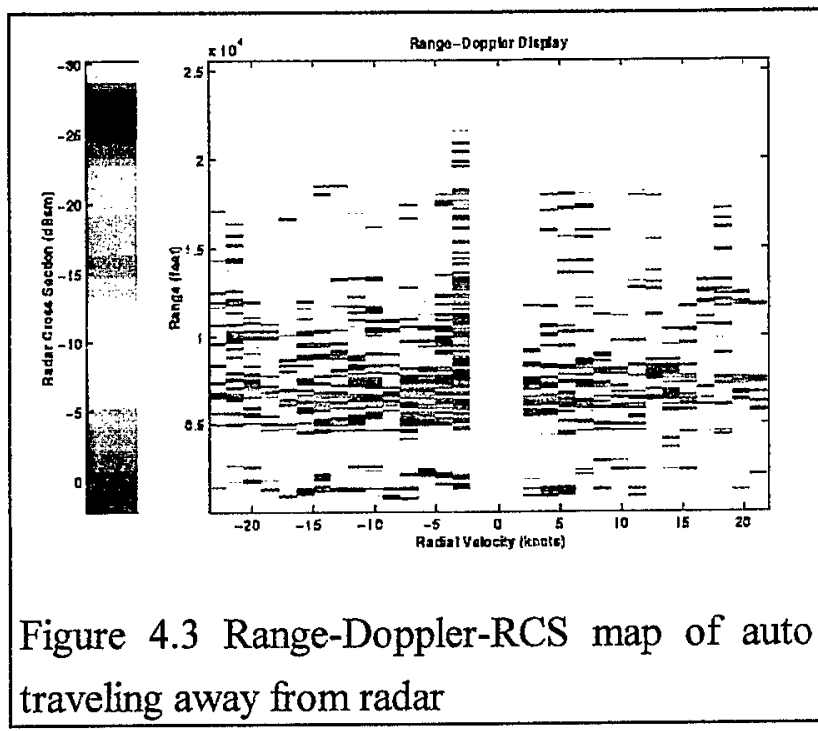
return located at $\sim 12000$ feet and 21 knots. The effective radar cross section of the vehicle was estimated at $1.7 \mathrm{dBsm}$. As noted in earlier measurements [9], the measured radar cross section from the test vehicle ranged from $-5 \mathrm{dBsm}$ to $+2 \mathrm{dBsm}$, with stronger returns from the rear of the vehicle. The returns near 0 knots are primarily clutter residue. As discussed in [9], the radar cross sections of the nearby town barn and houses were about $12 \mathrm{dBsm}$. The returns spread in range and velocity around 7000 feet also represent a combination of clutter residue and noise from a stronger return from a wooded hill. The signals near the auto's range and within a few knots in velocity are probably multipath returns off the houses and the town barn.

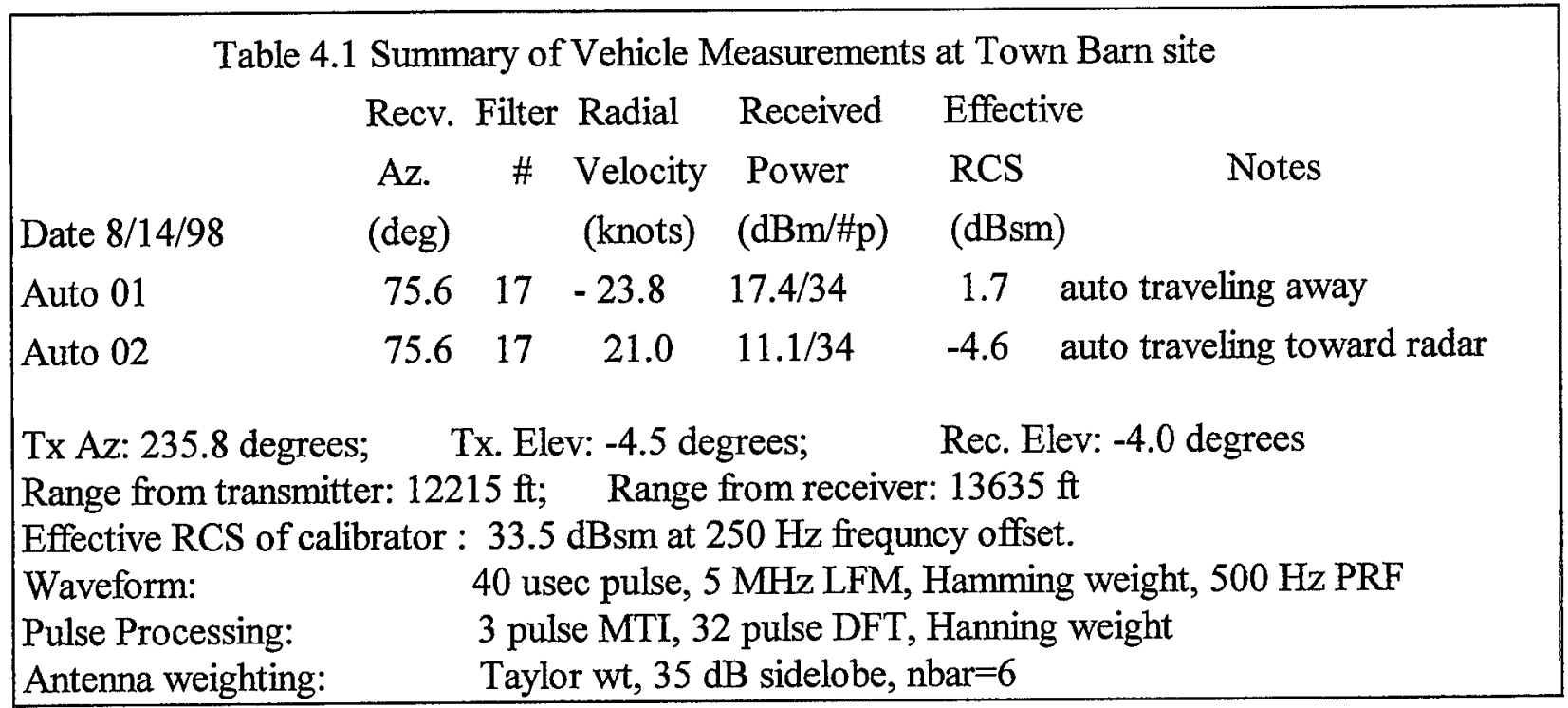




\subsection{Vehicle Measurements at the Farrell Road Site}

The Farrell sites provided a varying degree of screening by a mixture of branches, leaves and tree trunks. The angular difference between the bistatic bisector and the velocity vector of vehicles traveling on this road is 51 degrees. The radial velocity component of vehicles traveling down this road is approximately $62.9 \%$ of the vehicles true velocity or a nominal 16.4 knots for the $30 \mathrm{mph}$ test vehicle. The path of the vehicle through these sites is a straight line and the bistatic angle and the viewing geometry of the vehicle changed less than 5 degrees over the nine sites.

Table 4.2 summarizes the measurement results for the vehicle moving away from the bistatic radar. The table lists the Doppler filter where the target was found and the radial velocity corresponding to the center of that filter. The measurements in June and July used only a DFT in the processing while the August measurements used a 3 pulse MTI followed by a 32 pulse DFT.

Figure 4.4 presents the processed return from the vehicle at the reference site. The small RCS of the test vehicle limits the amount of attenuation that the vehicle can be reliably detected in. The target return is at 5100 feet and -18 knots. The white band centered around zero Hertz extends over the filters excluded from the MTI display compensation discussed earlier. The clutter from the terrain and vegetation in this region is about $10 \mathrm{dBsm}$ with stronger clutter at a range of about 10000 feet. The weak signals at the closer and farther ranges represent noise and clutter residue that is enhanced by the range and MTI normalization used. A Hanning weighting is used
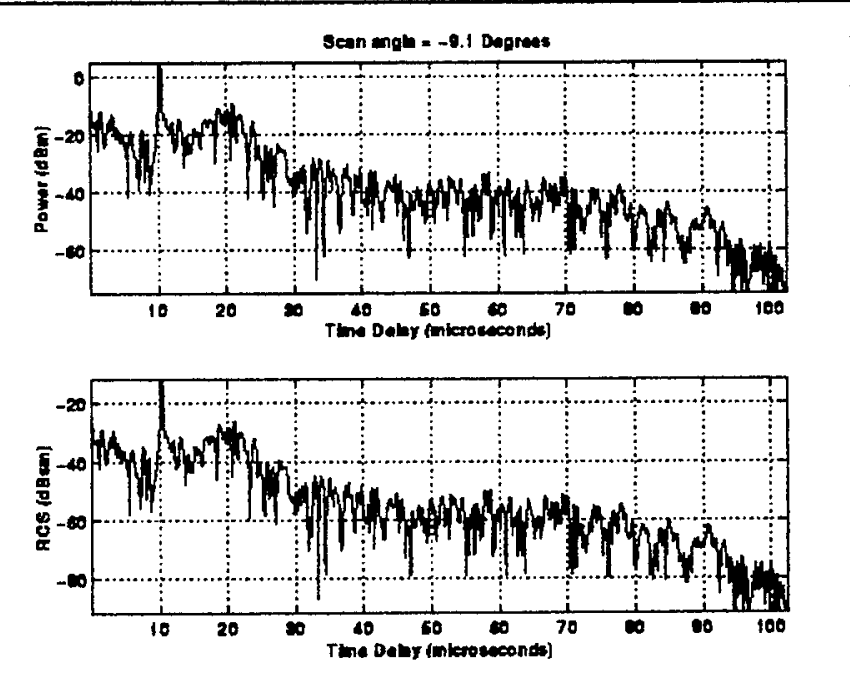

Figure 4.4 Power and effective RCS from vehicle in filter 7 of 16 . (Auto $0,6 / 24 / 98$ ) to reduce any target Doppler sidelobes well below noise. The signals at the target range and extending over several Doppler filters represent multipath returns from the target and the foliage [19].

The problem was complicated by a spurious interference that can be seen at 16 knots, -2 knots and +14 knots in Figure 4.5. This spurious interference changed in frequency and amplitude during the experiment and presented a decrease in usable sensitivity. This is apparent in Figure 4.6 where the target is not much 
larger than a background that should have been $10 \mathrm{~dB}$ less and closer to the thermal noise level. Estimates of the target's effective RCS depended on a priori knowledge of the target's rangeDoppler cell and an analysis of the corresponding range profile. Figures 4.7 and 4.8 present scatter plots of the data in Table 4.2.

The two-way attenuation measurements performed with the calibrator and presented in Chapter 3 showed attenuation values over $40 \mathrm{~dB}$. It was expected that the vehicle backscatter would not be detectable at locations with such high direct path attenuation. However, the vehicle return was detected at these locations and measured to have a higher effective RCS than expected. One possible explanation for this observation is that the signal path passing through the attenuating forest is not the only path for illuminating energy. As noted in the Chapter 3, the direct signal energy at locations with the highest foliage attenuation was equaled or exceeded by multipath energy from vegetation down the road that was directly illuminated. Therefore, at least for some geometries, the detection of moving vehicles screened by foliage can be better than that predicted by using direct path attenuation values.

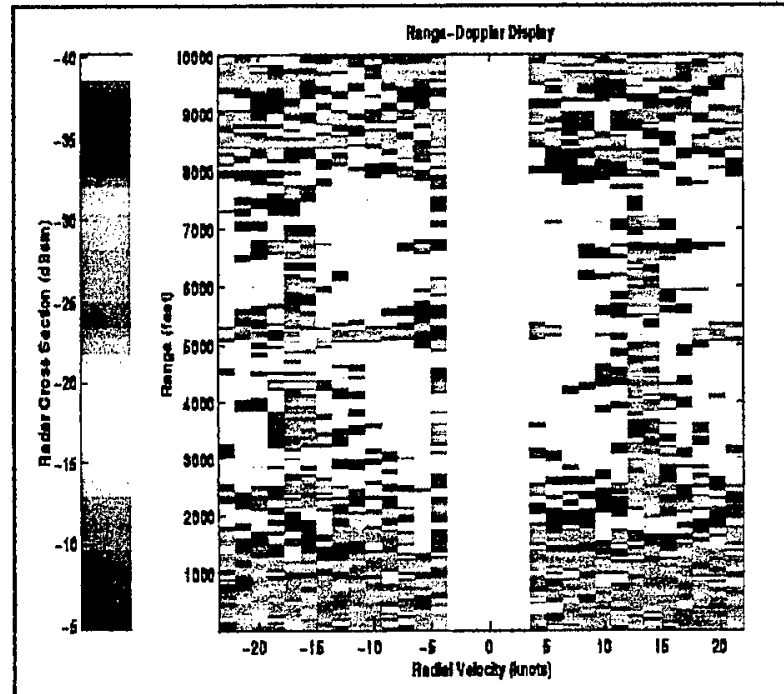

Figure 4.5 Range Doppler display of vehicle return at the reference site. (Auto 0, 8/19/98)

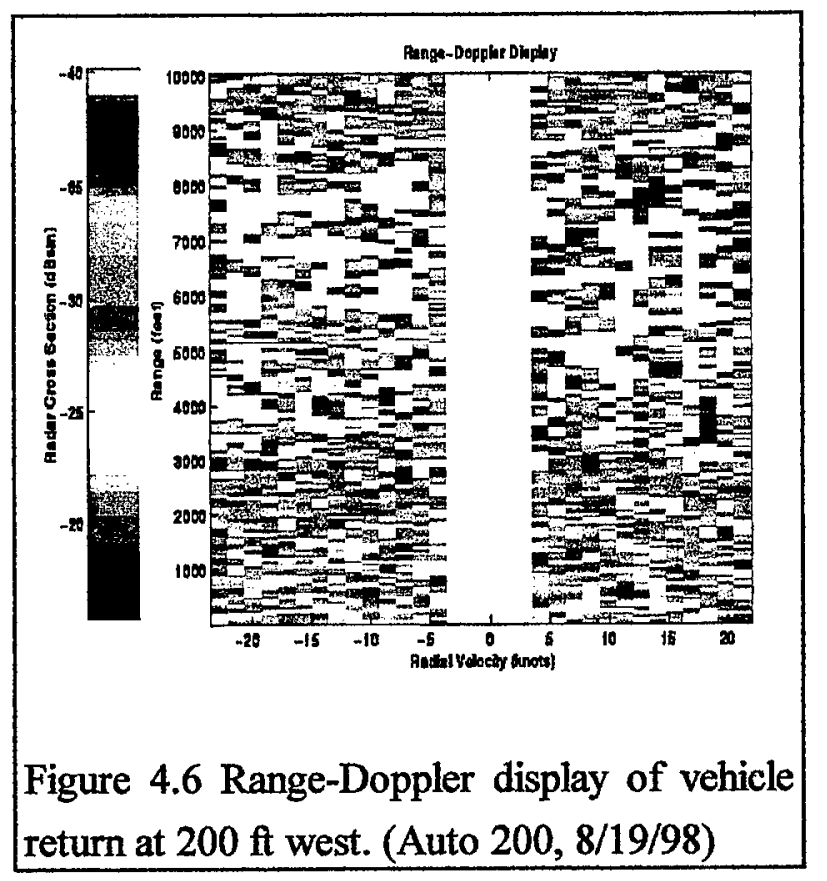


Table 4.2 Summary of Screened Vehicle Measurements at Farrell Road

Filter Radial Received Effective \#/DFT Velocity Power RCS

(knots) $\quad(\mathrm{dBm} / \mathrm{Hp}) \quad(\mathrm{dBsm})$

Date 6/24/98

Auto 0

$\begin{array}{llll}7 / 16 & -19.6 & 2.6 / 16 & -17.0\end{array}$

Auto 0

$\begin{array}{llll}6 / 16 & -16.8 & 8.2 / 16 & -11.5\end{array}$

Auto 50

$\begin{array}{llll}9 / 16 & -25.2 & 1.8 / 16 & -17.9\end{array}$

Auto 50

$\begin{array}{llll}8 / 16 & -22.4 & 0.6 / 16 & -18.9\end{array}$

Auto 100

$\begin{array}{llll}9 / 16 & -25.2 & -0.7 / 16 & -20.4\end{array}$

Auto 100

$\begin{array}{llll}7 / 16 & -10.6 & 2.9 / 16 & -16.7\end{array}$

Auto 150

$\begin{array}{llll}9 / 16 & -25.2 & 0.7 / 16 & -19.0\end{array}$

Auto 200

$7 / 16$

$-19.6 \quad-1.6 / 16$

$-21.3$

Date: 7/01/98

Auto 0

$\begin{array}{lll}46 / 126 & -16.4 & 12.0 / 128\end{array}$

$-18.1$

Auto 100

$49 / 126$

$1.2 / 128$

$-29.4$

Auto 200

$\begin{array}{lll}51 / 126 & -18.4 & 2.7 / 128\end{array}$

$-28.2$

Date: $8 / 14 / 98$

Auto 0

$12 / 32 \quad-16.8$

$8.8 / 34$

$-10.9$

Auto 50

$12 / 32$

$-16.8$

$11.0 / 34$

$-8.7$

Auto 100

$14 / 32$

$-18.2$

$-2.7 / 34$

$-22.4$

Auto 200

$13 / 3$

$-18.2$

$3.7 / 34$

$-16.0$

Auto 300

$14 / 32$

$-19.6$

$1.5 / 34$

$-18.2$

Auto 400

$13 / 32$

$-18.2$

$6.0 / 34$

$-13.7$

Date 8/19/98

Auto 0

Auto 100

$\begin{array}{llll}13 / 32 & -18.2 & 14.3 / 34 & -4.6\end{array}$

Auto 200

$13 / 3$

$-18.2$

$-0.3 / 34 \quad-19.1$

$\begin{array}{llll}13 / 32 & -18.2 & 6.3 / 34 & -12.6\end{array}$

Auto 300

$15 / 32$

$-1.2 / 34 \quad-20.1$

Auto 400

$15 / 32 \quad-21.0$

$-1.7 / 34 \quad-20.7$ 

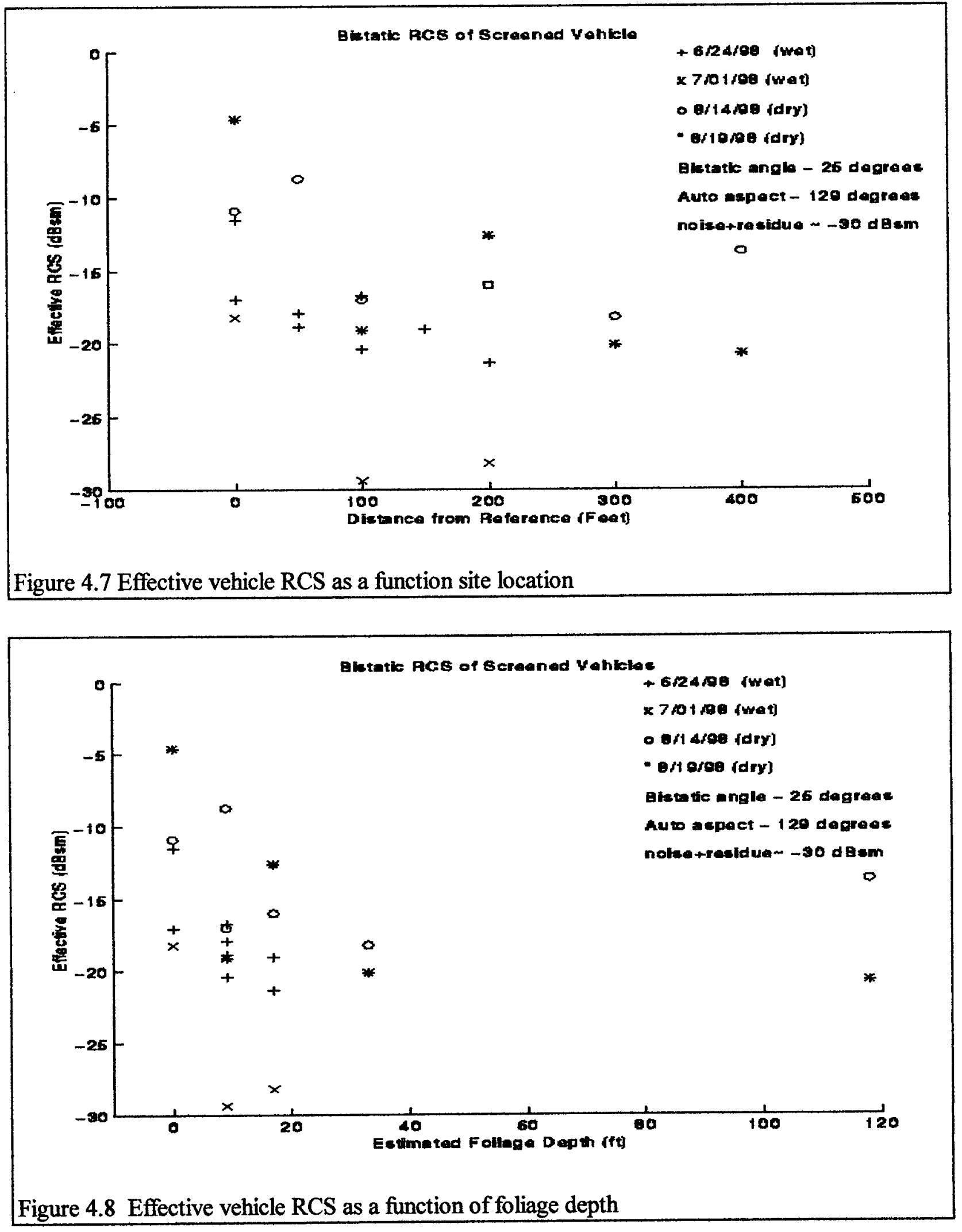


\subsection{Vehicle Measurements at the Newport Road Site}

The Newport road site provided an unscreened site, three sites that were optically screened by light brush and canopy and one screened site screened by several trees and dense brush. The angular difference between the bistatic bisector and the velocity vector of vehicles traveling on this road is 68 degrees. The radial velocity component of vehicles traveling down this road is approximately $37.5 \%$ of the vehicles true velocity or 9.6 knots for the $30 \mathrm{mph}$ test vehicle. The clutter from the terrain and vegetation in this region is about $10 \mathrm{dBsm}$.

Table 4.3 presents the measurements of the vehicle return at the reference location and the two higher attenuation sites east of the reference location. The system sensitivity was reduced by a low level interference from an unknown source. Figure 4.9 presents the recorded power and estimated radar cross of the vehicle in the 6th Doppler filter centered at 9.6 knots.

Figure 4.10 presents a range-Doppler presentation of the target at the reference site. The target is located at about 4000 feet in the 9.6 knot Doppler filter. A Hanning weight was used in the Doppler filter to reduce the sidelobe response from the target to a level below the noise thermal level. The weaker signals extending over several Doppler filters at the target's range represent the single and multiple bounce multipath returns of the target's backscatter [19]. The weak signals centered around the 10000 foot delay corresponds to the residue from the strong backscatter of a wooded hill. The weak signals linearly increasing in Doppler from 0 to 10000 feet represent the unknown interference that limited the sensitivity of the measurements.

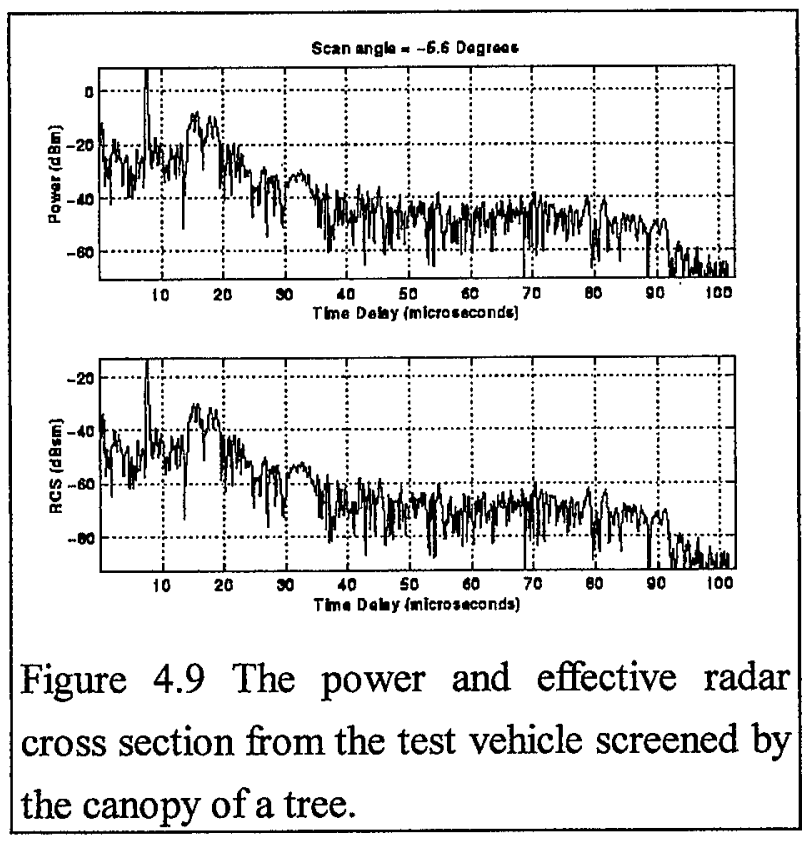

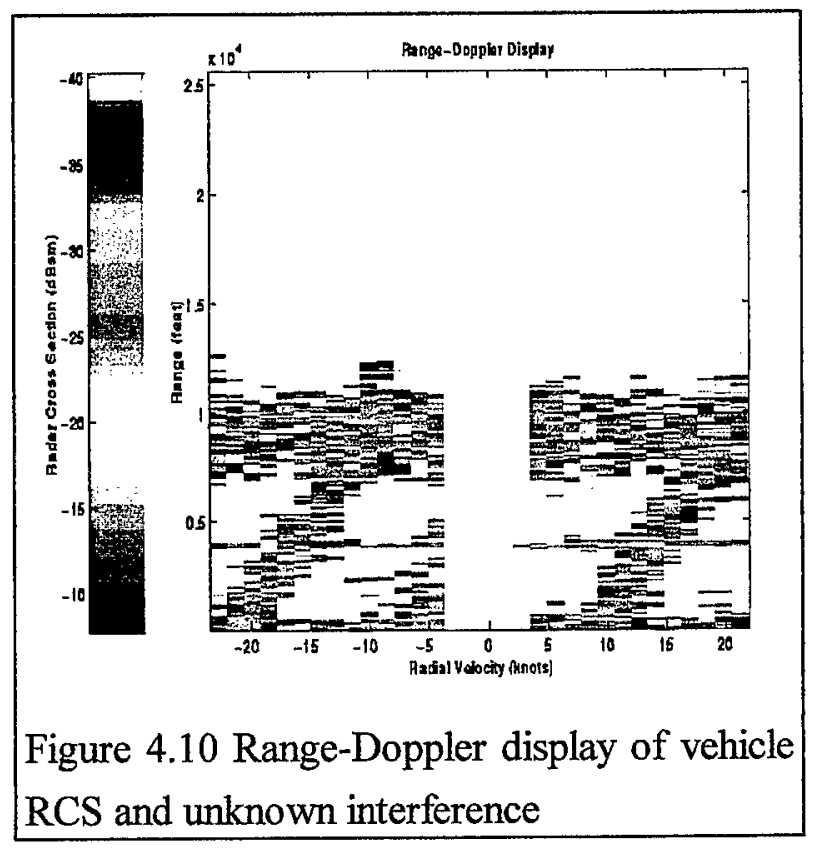


Table 4.3 Summary of Screened Vehicle Measurements at Newport road (site C).

$\begin{array}{llcc}\text { Filter } & \text { Radial } & \text { Received } & \text { Effective } \\ \# / \text { max } & \text { Velocity } & \text { Power } & \text { RCS } \\ & \text { (knots) } & (\mathrm{dBm} / \# \mathrm{p}) & (\mathrm{dBsm})\end{array}$

Date $8 / 14 / 98$

reference

$26 / 32$

\section{5}

$6.4 / 34$

$-7.1$

$100 \mathrm{E}$

$25 / 32$

10.0

$2.8 / 34$

$-12.5$

$200 \mathrm{E}$ target not detected $\#$

Date $8 / 19 / 98$

reference

$26 / 32$

8.5

$100 \mathrm{E}$

$25 / 32$

10.0

$9.0 / 34$

$-5.1$

$200 \mathrm{E}$

target not detected $\#$

* azimuth angles are local measurements logged for reference

\# masked by interference

Range from transmitter: 5030 feet

Range from receiver: 4875 feet

Transmitter Elevation angle: -6.5 degrees

Effective RCS of calibrator $=33.5 \mathrm{dBsm}$ at $250 \mathrm{~Hz}$.

Waveform: 40 usec pulse, $5 \mathrm{MHz}$ LFM, Hamming weight, $500 \mathrm{~Hz}$ PRF

Pulse Processing: 3 pulse MTI, 126 pulse DFT, Hanning weight

Antenna weight: Taylor wt, $35 \mathrm{~dB}$ sidelobe, nbar $=6$ 


\subsection{VXI DSP PROCESSING SUBSYSTEM}

The existing system needed faster processing to perform as an experimental bistatic adjunct to an existing monostatic radar or to obtain the statistical measurements of the bistatic clutter, foliage attenuation and target detection tests discussed earlier in this report. In 1996 and early 1997, a review was performed of the existing DSP products to find a compatible VXI-based DSP subsystem. The DSP subsystem had to meet the following criteria:

* must occupy no more than one C-size VXI mainframe slot;

* provide a minimum of MFLOPS of peak processing power;

* provide a minimum of 4 MBytes of Global RAM expandable to 16 MBytes;

* must allow for transfer of control and data with the VXI Intel-based system controller;

${ }^{*}$ must accept high speed transfers to and from the other VXI modules via the XI Local Bus using Hewlett-Packard (HP) compliant transfer protocols;

* must include software development programs, utilities and libraries to allow for the development of $\mathrm{C} / \mathrm{C}++$ programs for the DSP under a PC/Windows-based operating system.

Many of the well-known DSP board vendors were contacted including Hewlett-Packard, Kinetic Systems Corp., Pentek and Spectrum Signal Processing. None of the vendors had an existing system that met all the above criterion. However, Spectrum had a product under development, the LeMans VX8, with preliminary specifications that were close to the above requirements [Appendix B-1]. Furthermore, Spectrum indicated that the VX8 was being developed as part of a teaming agreement with HP, implying that the system would be compatible with the HP protocols. This information was provided to AFRL with the recommendation that the Spectrum LeMans VX8 be given serious consideration once the final specifications were received.

AFRL subsequently purchased the LeMans VX8 board and associated software. Chapter 5.1 presents a description of this DSP subsystem, the DSP modules and the associated software development tools. Chapter 5.2 presents a discussion of the radar signal processing algorithms developed during this effort and provide information of the timing and throughput of the DSP system. Chapter 5.3 presents some of the difficulties incurred during the effort, some of the lessons learned and suggestions on the steps needed to fully integrate the DSP subsystem into the bistatic system. 


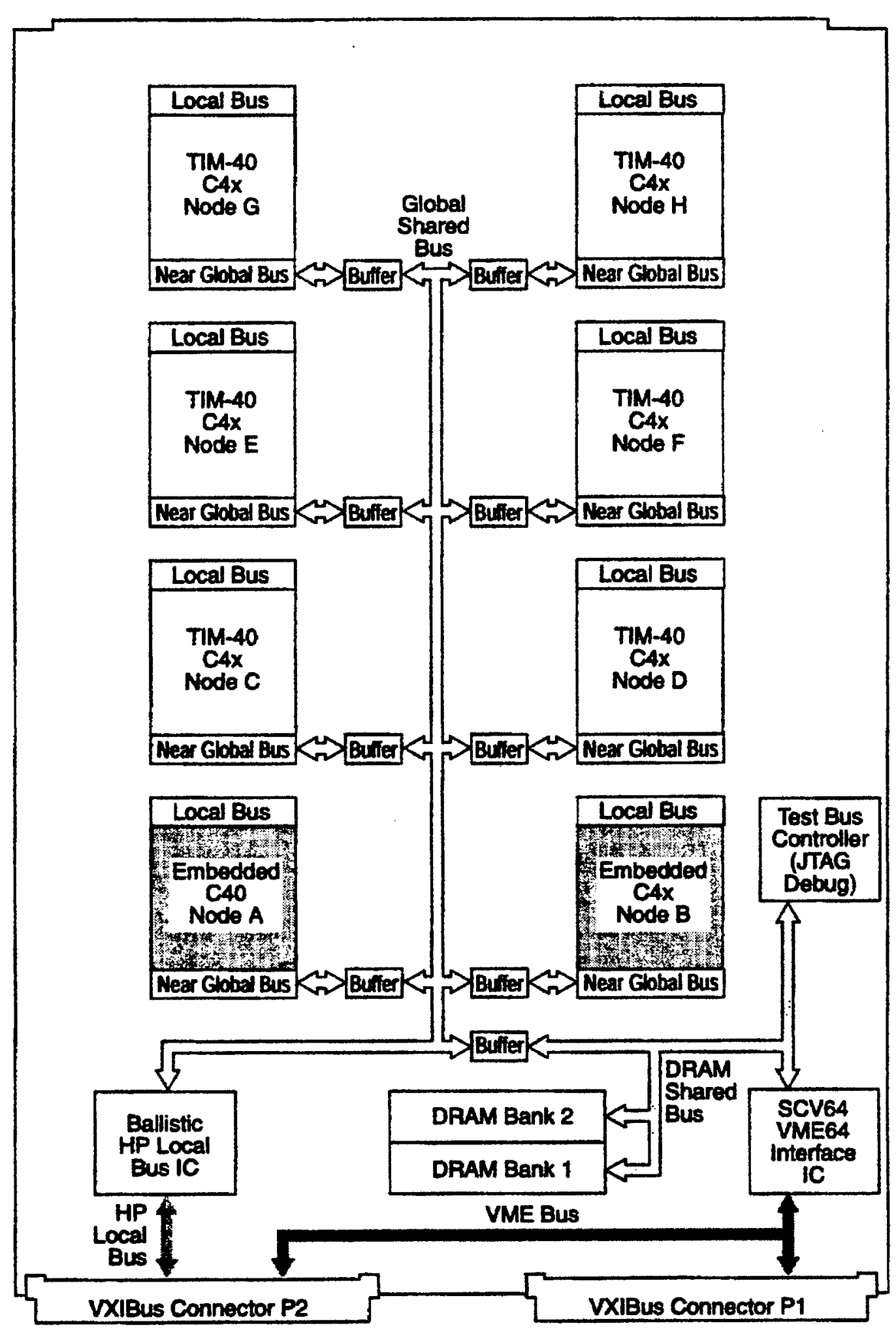

Figure 5.1 LeMans VX8 Bus Architecture ([23], Figure 4, p9) 


\subsection{LeMans VX8 Carrier Board}

The VXI-based DSP module provided by AFRL is the TMS320C4x-based LeMans VX8 system provided by Spectrum Signal Processing [20]. Spectrum's VX8 Carrier Board provides two on-board $60 \mathrm{MHz}$ TMS320C40 processors and 6 nodes for Texas Instruments' TIM-40 TMS320C4x modules [21]. Figure 5.1 shows the bus architecture interfacing each $\mathrm{C} 4 \mathrm{x}$ node, the dynamic random access memory (DRAM), the Ballistic HP local bus IC, the JTAG interface and the SCV64 VXIbus interface IC. The VX8 has a register based VXIbus interface. The VX8 can function as either a master or as a slave module on the VXIbus. The DRAM Shared Bus enables the VXIbus slave interface to access the DRAM, Test Bus Controller, and Global Shared Bus. It also allows a C4x DSP to access the DRAM, control registers, status registers and the SCV64 as a VXI master.

One reason for the choice of the LeMans VX8 board was its HP local bus interface which uses a high speed BALLISTIC chip. The interface includes a $1 \mathrm{k} \times 32$ bit FIFO and an intelligent DMA controller which allows a HP Local Bus master to write to the Near Global Memory (NGM) of any single C40 node or the NGM of the C44A processor of the dual C44 MDC44ST TIM-40 module. The DRAM is not accessible by the HP Local Bus Interface.

The VX8 embedded firmware uses a system definition file (SDF) to define the configuration of each module and the interface between the modules. Spectrum provided a SDF file for the basic module with examples showing how an SDF could be created for various configurations of the VX8. Figure 5.2 shows the SDF file for the existing system. The configuration information identifies the VISA base address, the definition and location of each type of C4x module in the system, the memory access and wait states of the global and local memory, the communication ports within the module as well as between the modules and the board's boot processor.

Each embedded node (Nodes A and B) provides the following features:

* One Local Bus memory bank of $128 \mathrm{~K}$ x 320 wait state SRAM;

* One Near Global Bus with a memory bank of $128 \mathrm{~K}$ x 32 0-wait state SRAM and a buffered interface to the Global Shared Bus;

* an interface to the Hewlett Packard (HP) Local Bus via the Global Shared Bus and a Ballistic IC;

* Access to the SCV64 interface to the VXIbus;

* one $32 \mathrm{~K}$ x 8 PEROM for booting or user programming. 


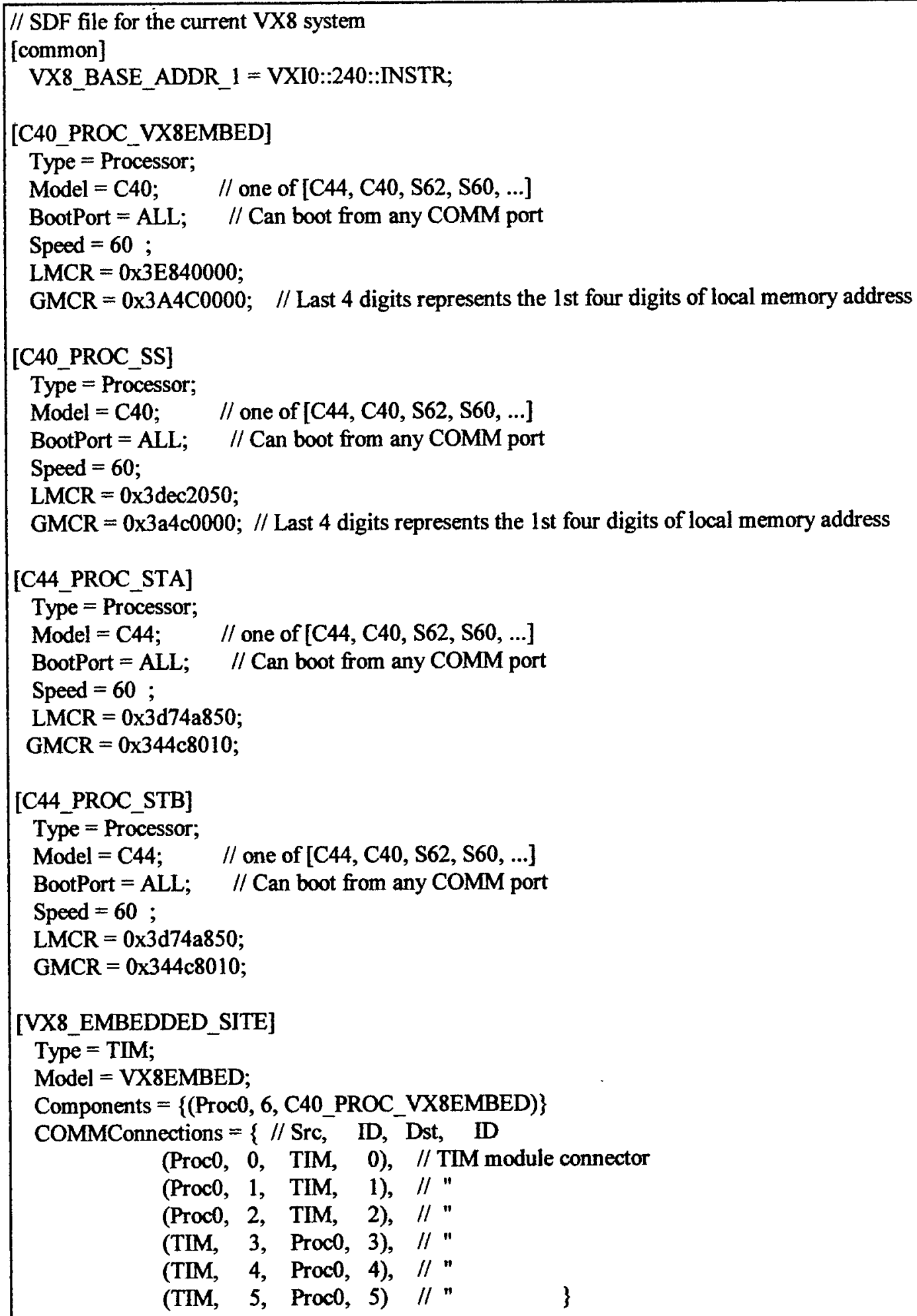

Figure 5.2 System Definition File (SDF) created for the existing VX8 Board 


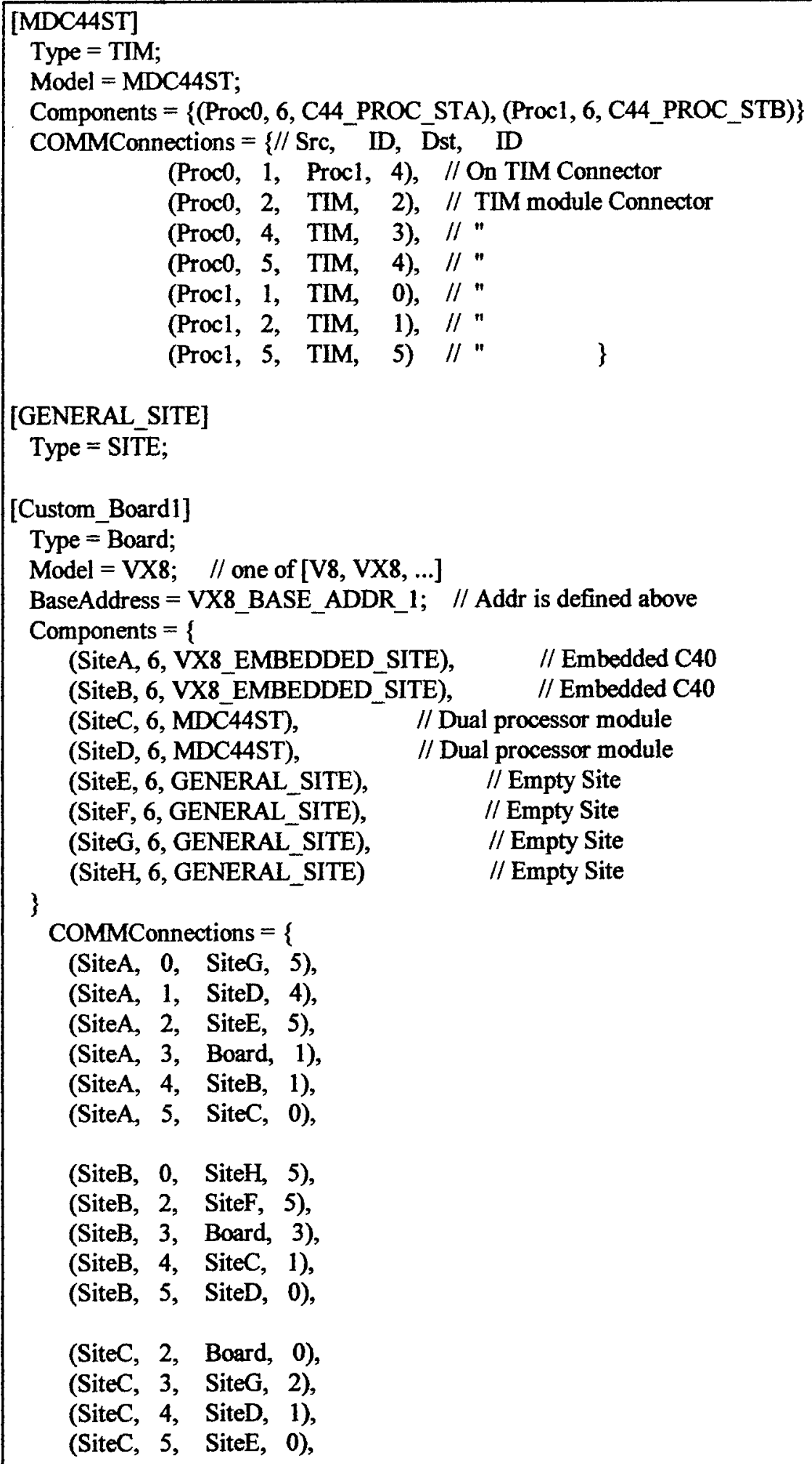

Figure 5.2 System Definition File (SDF) created for the existing VX8 Board (continued) 
(SiteC, 2, Board, 0),

(SiteC, 3, SiteG, 2),

(SiteC, 4, SiteD, 1),

(SiteC, 5, SiteE, 0),

(SiteD, 2, Board, 2),

(SiteD, 3, SiteH, 2),

(SiteD, 5, SiteF, 0),

(SiteE, 1, SiteH, 4),

(SiteE, 2, Board, 4),

(SiteE, 3, SiteG, 0),

(SiteE, 4, SiteF, 1),

(SiteF, 2, Board, 6),

(SiteF, 3, SiteH, 0),

(SiteF, 4, SiteG, 1),

(SiteG, 3, Board, 5),

(SiteG, 4, SiteH, 1),

(SiteH, 3, Board, 7) \}

[TestSystem]

Type = System;

Model = Development;

Components $=$

\{

(Board1, 8, Custom_Board1) // Standard base board. [Board1] section defines sites.

\}

// COMMConnections $=\{/ / \mathrm{Src}, \quad$ D, Dst, ID

// 3

[BootProc]

Board1:SiteA:Proc0 $=$ VX8_BASE_ADDR1;

[End]

Figure 5.2 System Definition File (SDF) created for the existing VX8 Board (continued) 
Node A has an additional 32K x 8 PEROM for the board's boot kernel while Node B has a DUART equipped with RS-232 drivers interfaced with the front panel RS-232 asynchronous serial ports. Figure 5.3 displays the architecture of Node A.

The VX8 module was also purchased with two Spectrum $60 \mathrm{MHz}$ MDC44ST Twin TMS320C44 TIM modules [43]. Spectrum's MDC44ST module is a single-width TIM-40 module equipped with two TMS320C44 DSPs. Each TMS320C44 DSP contains the following features:

* One 32K x 8 PEROM;

* One Local Bus memory bank of $128 \mathrm{~K}$ x 32 0-wait-state SRAM;

* One Global Bus memory bank of $128 \mathrm{~K}$ x 32 0-wait-state SRAM;

* Four communication ports.

Figure 5.4 presents a block diagram of the MDC44ST TIM-40 module. The two DSPs are designated as C44A and C44B. C44A has a global bus connector and a Global Page Register that allows it to access the full address range on the Global Connector and to provide an interface to the HP Local Bus. The C44B has no interface to the global bus connector and depends on its four

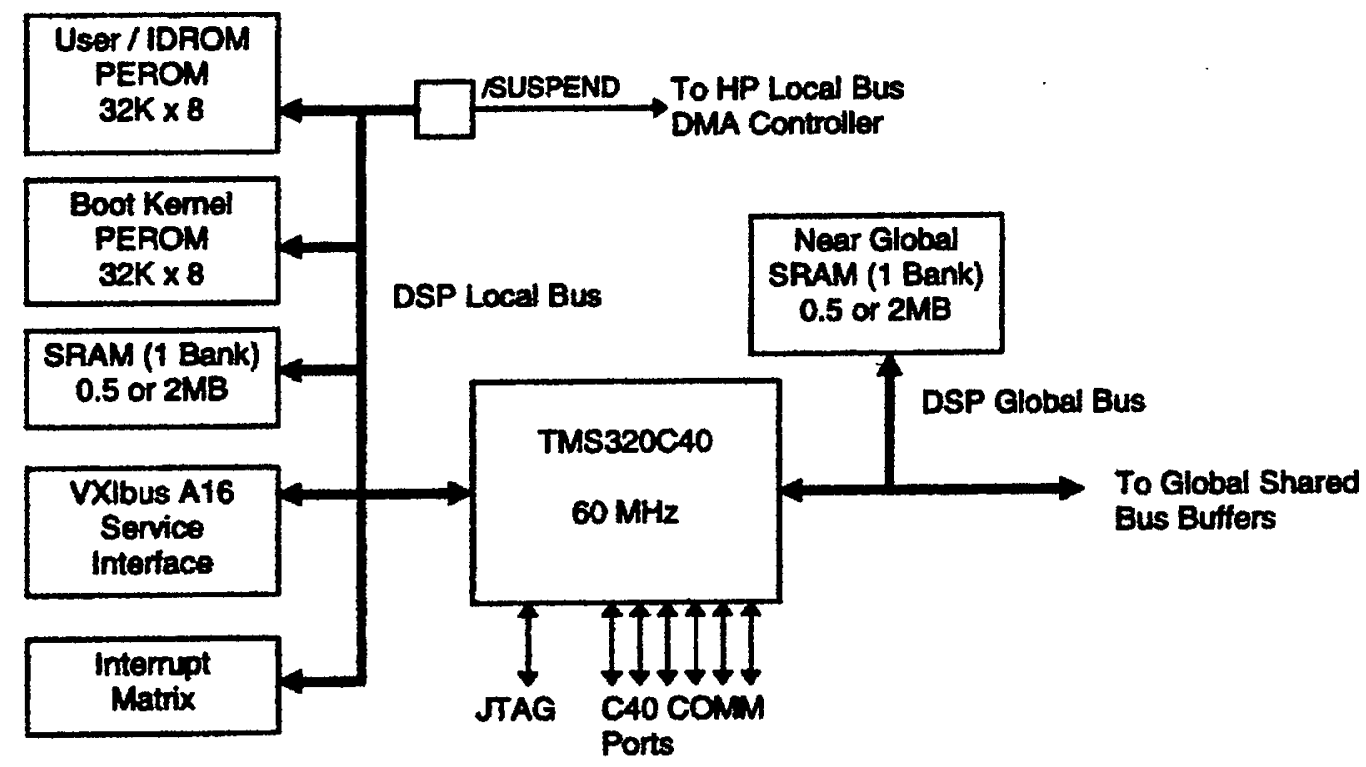

Figure 5.3 Embedded Node A architecture 


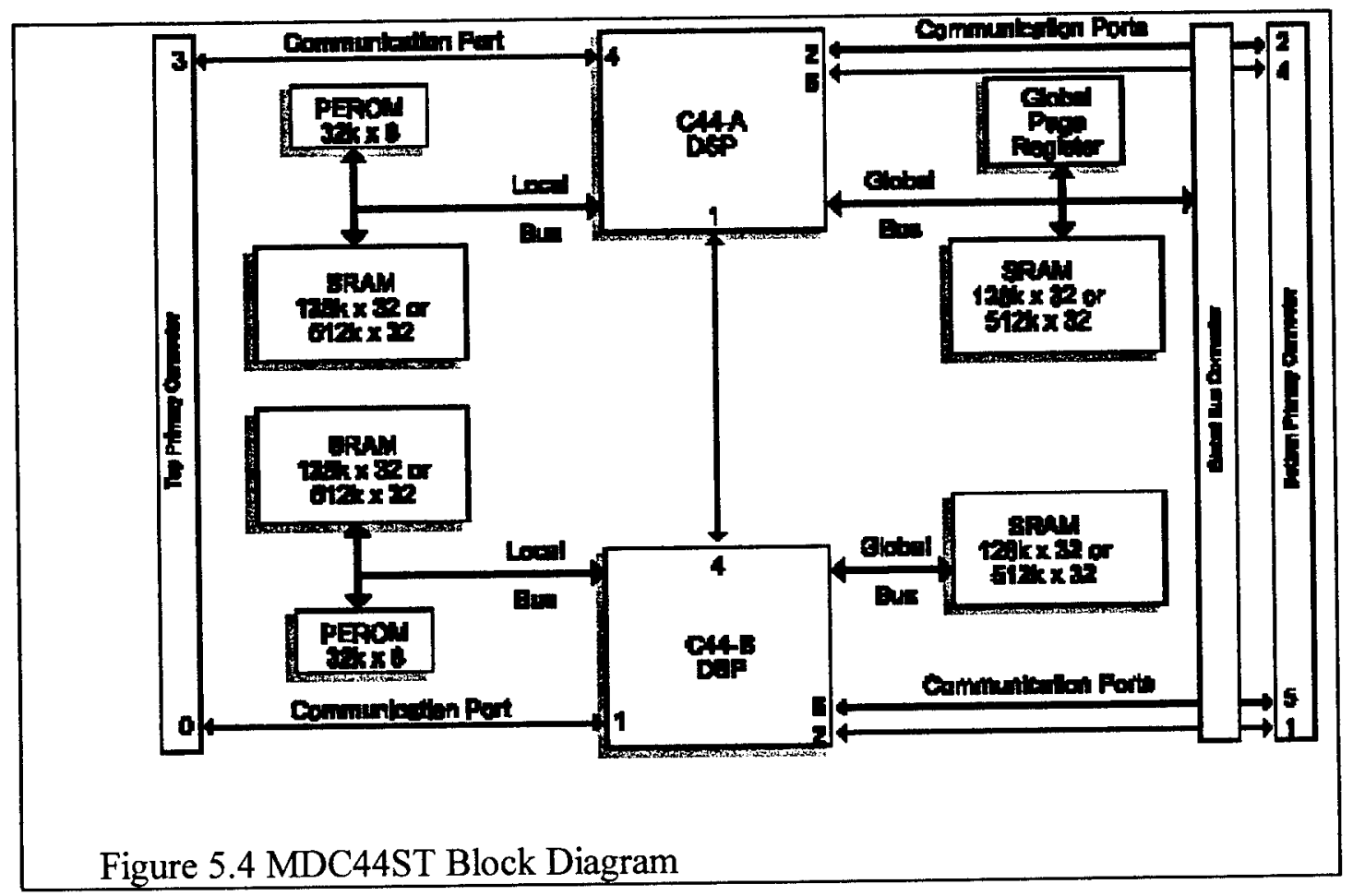

$20 \mathrm{MBps}$ parallel communication ports for interface to the C44A DSP and the other VX8 modules.

Figure 5.5 presents the Communication Port Routing used in the VX8 module. The C4x communication ports provide high speed parallel interface communications to other DSP's and I/O sources. Each Comm port is a The VX8 board has two quads (ABCD and EFGH) where each node has a dedicated Comm port to each of the other nodes. The ports are inherently bidirectional and point to point with no latency. Each COMM port can be used in unified or half duplex modes.

Figure 5.6 presents the number of timing cycles used to read from and write to the VX8 memory and registers. The $60 \mathrm{MHz}$ TMS320C4x DSP runs off the $30 \mathrm{MHz} H 1$ clock. A zero wait state read or write of a 32 bit word from internal SRAM requires one $33 \mathrm{nsec}$ timing cycle. Zero wait state external SRAM requires 1 cycle to read and 2 cycles to write. This corresponds to a read rate of $120 \mathrm{MBytes} / \mathrm{sec}$ and a write rate of $60 \mathrm{MBytes} / \mathrm{sec}$. Since all single precision data types from character to floating word occupy $32 \mathrm{bits}$, it is useful to talk about these transfer rates in terms of $30 \mathrm{MWords} / \mathrm{sec}$ for reading and $15 \mathrm{MWords} / \mathrm{sec}$ for writing to an external SRAM. Rates for other memory accesses are the following:

* Another DSP's NGM - 10 Mwords/sec R/W; 


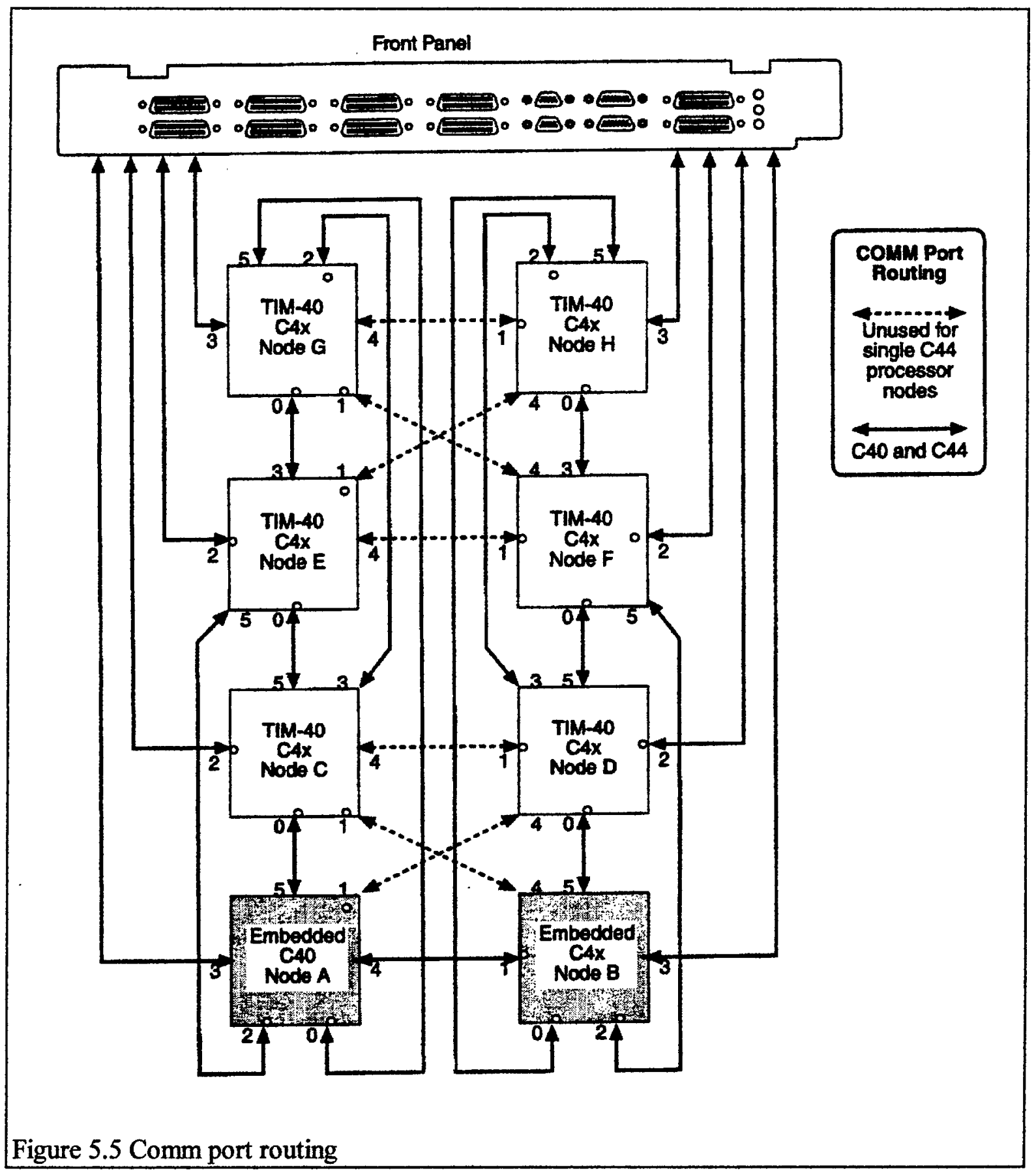

* DSP's NGM from VXIbus - $6 \mathrm{MWords} / \mathrm{sec}$;

* DRAM from C4x - 6 MWords/sec R and 5 MWords/sec W;

* DRAM from VXIbus - 4.25 MWords/sec R/W 


\begin{tabular}{|c|c|c|c|}
\hline \multicolumn{4}{|c|}{ Table 5.1 VX8 Timing Cycles [20, page 85$]$} \\
\hline $\begin{array}{l}\text { Access Type } \\
\text { Type }\end{array}$ & $\begin{array}{c}\text { Local or } \\
\text { Global Bus } \\
\text { (from } \mathrm{C} 4 \mathrm{x} \text { ) }\end{array}$ & $\begin{array}{c}\text { Global } \\
\text { Shared Bus } \\
\text { (from C4x) }\end{array}$ & $\begin{array}{c}\text { Slave } \\
\text { Access } \\
\text { (from VXIbus) }\end{array}$ \\
\hline $\begin{array}{l}\text { Local Bus } \\
\text { PEROM }\end{array}$ & $\begin{array}{l}5 \text { Read } \\
7 \text { Write }\end{array}$ & N/A & $\mathrm{N} / \mathrm{A}$ \\
\hline $\begin{array}{l}\text { Local Bus } \\
\text { DUART }\end{array}$ & $4 \mathrm{R} / \mathrm{W}$ & N/A & N/A \\
\hline VXIbus A16 & $2 \mathrm{Read}$ & N/A & N/A \\
\hline Registers & 3 Write & & \\
\hline $\begin{array}{l}\text { IIOF2 Int. } \\
\text { Control Reg. }\end{array}$ & 3 Write & N/A & N/A \\
\hline $\begin{array}{l}\text { /SUSPEND } \\
\text { Register }\end{array}$ & 3 Write & N/A & N/A \\
\hline $\begin{array}{l}\text { Local } \\
\text { SRAM }\end{array}$ & $\begin{array}{l}1 \text { Read } \\
2 \text { Write }\end{array}$ & N/A & N/A \\
\hline $\begin{array}{l}\text { Global } \\
\text { SRAM }\end{array}$ & $\begin{array}{l}1 \text { Read } \\
2 \text { Write }\end{array}$ & $\begin{array}{l}3 \text { Read } \\
3 \text { Write }\end{array}$ & $\begin{array}{l}5 \text { Read } \\
5 \text { Write }\end{array}$ \\
\hline $\begin{array}{l}\text { Internal } \\
\text { SRAM }\end{array}$ & $\begin{array}{l}1 \text { Read } \\
1 \text { Read }\end{array}$ & N/A & N/A \\
\hline $\begin{array}{l}\text { Global } \\
\text { DRAM }\end{array}$ & $\mathrm{N} / \mathrm{A}$ & $\begin{array}{l}5 \text { Read } \\
6 \text { Write }\end{array}$ & $\begin{array}{l}6 \text { Read } \\
6 \text { Write }\end{array}$ \\
\hline $\begin{array}{l}\text { HP Local } \\
\text { Bus Reg. }\end{array}$ & N/A & $\begin{array}{l}1 \text { Read } \\
1 \text { Write }\end{array}$ & N/A \\
\hline
\end{tabular}




\subsubsection{DSP Software Development Tools}

Several software tools for the development of the DSP code were provided by AFRL. First, Spectrum provided two $\mathrm{C}$ language libraries for use with their unique VX8 board architecture. Other tools include TI's TMS320 $3 \mathrm{x} / \mathrm{C} 4 \mathrm{x}$ Code Generation Tools, Code Composer ${ }^{\mathrm{TM}}$ development software, a JTAG interface by White Mountain and precompiled DSP math routines by Sinectonalysis.

\subsubsection{VX8 Software Libraries}

Spectrum provides a VX8 C4x Support Software Library for use by the DSP modules and a VX8 Host Software Library for use on a VISA-based host computer.

\section{VX8 C4x Support Library [23]}

The VX8 C4x Support Software Library is divided into 5 distinct modules. The A16 Control Module is part of a larger group of functions that make up the Node A Boot Kernel PEROM Code. This module is associated with the initialization sequence of the board and the servicing of the A16 Interrupt Service Routine (BOOT_IIOFIsr) used in the VX8 board.

The Global Bus Interface Module provides the functions used for all transactions between the C4x node and an external resource on the Global Shared and DRAM Shared buses. These resources include registers, another Node's Near Global Memory, DRAM, the HP Local Bus and the VXIbus. This module provides the interface functions only for the single $\mathrm{C} 40$ modules and the C44A processor of the dual C44 MDC44ST module. The C44B module has no direct interface with the Global Bus.

The VXIbus Interface Module provides the capability for the C4x DSP nodes to write to the SCV64 for initialization, setup of the VXIbus Mastering, VXIbus Interrupt Generation and Handling, and setup of the SCV64 DMA Controller. This module provides the interface functions only for the single $\mathrm{C} 40$ modules and the C44A processor of the dual C44 MDC44ST module. The C44B module has no direct interface with the VXIbus Bus.

The HP Local Bus Interface Module contains functions and macros for performing DMA transfers of data between a C4x NGM and the HP Local Bus. This module provides the interface functions only for the single $\mathrm{C} 40$ modules and the C44A processor of the dual C44 MDC44ST module. The C44B module has no direct interface with the HP Local Bus. 
The DUART Module provides functions and macros to use the 2 channel $16 \mathrm{C} 550$ style UART on the VX8 Carrier Board. This module provides the interface functions only for the single C40 modules and the C44B processor of the dual C44 MDC44ST module.

\section{VX8 Host Software Library}

The VX8 Host Software Library provides a VX8 Instrument Driver and a limited number of routines for writing to and from the VX8 board and the C4x modules. The Instrument Driver supports the $\mathrm{I} / \mathrm{O}$ libraries provided in the VXIpnp Alliance Virtual Instrument Software Architecture (VISA). The routines include functions to open and close sessions, assess VX8 systems, load application software and transfer blocks of data to and from the DRAM and NGM of the $\mathrm{C} 4 \mathrm{x}$ modules.

A Load Definition File (LDF) (Figure 5.6) defines the software configuration of the VX8 system. LDF contains the paths and filenames for the C4x DSP executables that are to be loaded by the Instrument Driver.

[Files]

Board1:SiteA:Proc0 = boot_io.out; // Embedded TIM A

Board1:SiteB:Proc0 = beamform.out; // Embedded TIM B

Board1:SiteC:Proc0 = adc2dspc.out; // Embedded TIM C0

Board1:SiteC:Proc1 = filt_cb.out; // Embedded TIM C1

Board1:SiteD:Proc0 = adc2dspd.out; // Embedded TIM C0

Board1:SiteD:Proc1 = filt_db.out; // Embedded TIM C1

[end]

Figure 5.6 Example of an Load Definition File for the existing VX8 system. 


\subsubsection{TI TMS320C3x/C4x Code Generation Tools ${ }^{1}$}

The Texas Instruments TMS320C3x/C4x Code Generation Tools provides a DSP codedevelopment environment on a PC running Windows NT or Windows 95. The release 5.00 version of these tools includes an optimizing $\mathrm{C}$ compiler and a full set of assembly language tools for developing and manipulating assembly language and object (executable) code.

The compiler tools include the following:

* Parser

* Optimizer

* Code generator

* Interlist and library-build utilities

The assembly language tools are composed of the following:

* Assembler

* Archiver

* Linker

* Absolute and cross-reference lister

${ }^{*}$ Hex conversion utility

The assembly and compiler tools can be used separately from other programs via command line in a DOS console window. The tools are also compatible with Windows-based development packages such as Code Composer ${ }^{\mathrm{TM}}$, a GUI-based development package developed by GO DSP, a Texas Instruments Company.

The tools do not include a debugging or profiling capability. For this effort, these capabilities were provided by Code Composer and a JTAG interface provided by White Mountain DSP.

1 TMS320C3x/4x Code Generation Tools (Release 5.00), Getting Started Guide, Texas Instruments Incorporated, 1997, www.ti.com, (document: spru119B.pdf) 


\subsubsection{GO DSP Code Composer ${ }^{2}$}

Code Composer ${ }^{\mathrm{TM}}$ is a comprehensive Integrated Development Environment (IDE) developed by GO DSP, a Texas Instruments Company. Code Composer allows DSP code designers to edit, build, manage projects, debug and profile from a single integrated application in both UNIX (Sun Solaris) and Windows (95 and

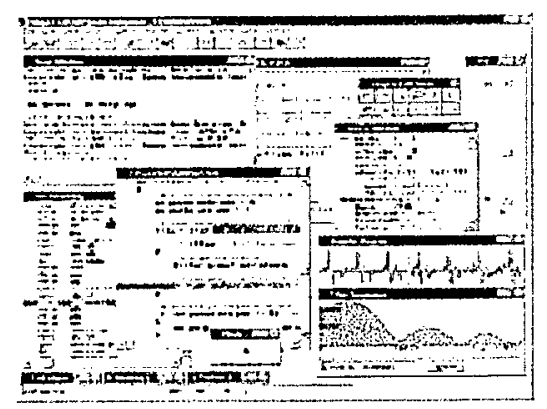

Figure 5.8 Code Composer Graphic Interface NT) environments. In addition, the IDE lets users compile in the background as well as perform graphical signal analysis (including eye diagrams, constellation diagrams, FFT waterfalls, image displays, etc.) and perform file $\mathrm{I} / \mathrm{O}$, debug multiple processors, customize the IDE via GEL, a Cinterpretive scripting language, and much more. A Visual Project Management System allows users to easily visualize, access and manipulate all project files and keeps track of all dependencies. Code Composer includes the following features:

* Integrated Development Environment (IDE)

* Visual Project Management System

* Leading edge GUI

* Full-featured debugging including assembly source level debugging

* File I/O with advanced triggering

* Signal probe points

* Advanced graphical signal analysis

* Multiprocessing

* Interactive profiling and analysis

* Open Plug-in architecture

* Quick Watch for instant access to variables

* C-Interpretive scripting language to add your own functionality

2 GO DSP Corporation, 260 Richmond St. W., Suite 501, toronto, Ontario, Canada M5V 1W5, (416)-599-6868, email:www.go-dsp.com 


\subsubsection{Mountain-510 Universal DSP} Emulator- PC-AT/ISA ${ }^{3}$

The White Mountain DSP Mountain-510 Universal Emulator provides a C/assembly source level debugging capability for the entire $\mathrm{C} 2 \mathrm{xx}, \mathrm{C} 3 \mathrm{x}, \mathrm{C} 4 \mathrm{x}, \mathrm{C} 5 \mathrm{x}, \mathrm{C} 54 \mathrm{x}, \mathrm{C} 6 \mathrm{x}$ and $\mathrm{C} 8 \mathrm{x}$ family. TI's TMS470 MCU is also supported.

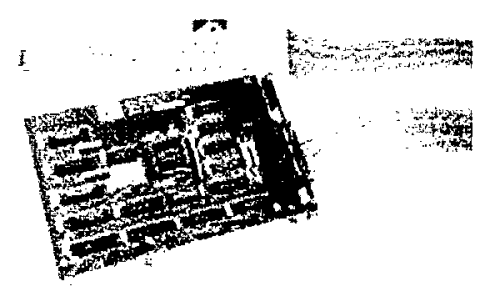

The Mountain-510's scan path connection controls the DSPs within the targeted applications as well as both internal and external memory. Debugger options for the Mountain-510 include TI's Code Composer environments or Allant's ASPEXTM. Some of the additional features of this universal emulator and debug environment are the following:

* Low profile 16 bit PC-AT hosted emulator card for 386, 486, and Pentium class machines

* Rugged high-speed 3V/5V scan-based flexible emulator cable and pod (1 meter)

* Interchangeable pod connection and ejector latched allows for easy transition from MPSD to JTAG DSPs

\subsubsection{Sinectoanalysis Math Libraries ${ }^{4}$}

Three DSP math libraries developed by Sinectoanalysis were provided for the development of the DSP software. The Cblas123 Library provides routines to provide common real and complex vector and matrix operations. The DSP/Vector Library extends the real and complex vector routines to perform common algorithms for signal processing such as FFT and correlation routines. The STD/Mathlib Library provides power, exponential and trigonometric functions. These functions were written in TMS320C40 assembly language to take advantage of the features inherent in the pipe-lined TMS320 architecture. The library is support under the development environment using the TI TMS320C4x C compiler and assembler and Go DSP's Code Composer.

\footnotetext{
${ }^{3}$ White Mountain DSP, 20 Cotton Road, Nashua, NH 03063, tel:(603) 883-2430 Fax: (603) 882-2655, e-mail: info@wmdsp.com,Copyright 01998

4 Sineconalysis, Inc., 24 Murray Rd., West Newton, MA 02165, e0mail: sinecto@clark.net, tel:617-894-8296
} 


\subsection{C4X DSP SIGNAL PROCESSING SOFTWARE}

Figure 5.10 presents the allocation of signal processing and other functions among the DSP modules. Figure 5.11 shows the timing of the parallel pipeline approach was used to implement the radar signal processing. Nodes $C$ and D are Spectrum MDS44ST TIMs, each with two TMS320C44 processors. In this discussion, the $\mathrm{C} 44 \mathrm{~A}$ processors are referenced as Node $\mathrm{C} 0$ and Node D0 while the $\mathrm{C} 44 \mathrm{~B}$ processors are referenced as Node $\mathrm{C} 1$ and Node D1.

The digitizer's first channel data is transferred via the HP Local Bus and the Global Shared Bus to the Near Global Memory (NGM) of Node C0. Node C0 performs the preprocessing and Digital I/Q on the data and transfers the data to the NGM of Node C1 via DMA 1 and high speed Comm port COMM 1. While Node $\mathrm{C} 0$ is processing the first channel data, the data from the second channel of the digitizer is transferred to the NGM of Node D0 where identical processing is taking place. After the data is transferred to Node CB, Node CA receives the data from the next odd digitizer channel. The process is repeated until data from all the digitizer channels have been completed.

Node $\mathrm{C} 1$ receives the data from $\mathrm{C} 0$ thru COMM 4. Node $\mathrm{C} 1$ then provides the rangeDoppler processing and transfers those range cells that were fully processed to the NGM of Node $B$ via DMA 2 and COMM 2. Node B integrates the range-Doppler data from the 16 channels in up to 9 beams. After the last channel has been integrated and the beams are fully formed, the data is converted from DSP floating point to IEEE floating point and transferred to DRAM. The host then performs a transfer of the data from DRAM to a file on the host.

Nodes $\mathrm{C} 1$ and $\mathrm{D} 1$ perform basic $\mathrm{I} / \mathrm{O}$ and preprocessing functions and have been programmed to operate with minimum deadtime. The signal processing functions have been allocated to the other nodes to provide each node with some extra time for future system expansion or the development of adaptive processing to meet future experimental needs. Node A is unique in that it is the only node that provides the system boot for the board and can dominate the Global Shared Bus activity for interrupt response. Therefore, much of the processing capacity of Node A was reserved for future use.

The following sections discuss the processing used in each module and present profiling result that demonstrate the effective MFLOPS and throughput obtained in the system. First the signal processing performed in the modules is discussed. Then the interfaces between the VX8, the VXI digitizers and the host are discussed. 


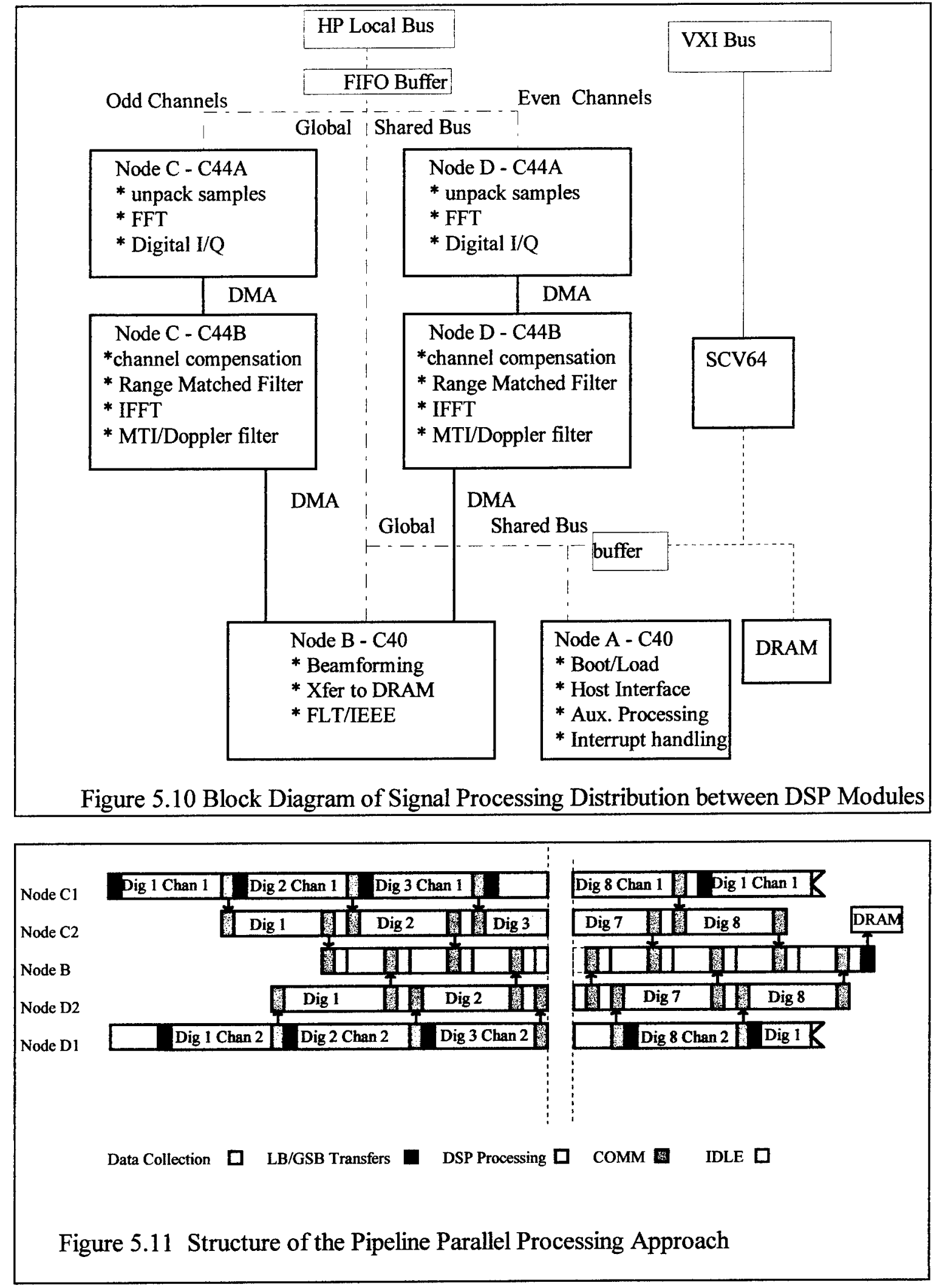




\subsubsection{Nodes C and D - Digital I/Q Demodulation}

Nodes $\mathrm{C}$ and D are dual C44 modules as described in Chapter 5.1.3. Within each dual C44 module are two C44 DSPs designated processor 0 and processor 1, each with its own global and local buses and Comm ports. Only the processor 0 has a direct interface with the Shared Global Bus and the HP Local Bus. Processor 1 depends on Comm ports for I/O to the other VX8 modules. Each global and local memory bank currently consists of $512 \mathrm{KBytes}$ of zero wait state SRAM with an expansion option of up to 2 MBytes. Since the C4x DSP allocates 32 bits for all single precision data types including character, integer and floating point word, this memory can be considered as $128 \mathrm{~K}$ words. Double precision floating point words are 40 bits wide.

Many of the DSP functions provide optimum speed when the two different buses or one bus and the internal RAM are used for the operands. These functions either do not allow "in-place" operation or provide a performance penalty when the same bus is used. Therefore, most of the Global and Local Memory blocks are defined for data storage and manipulation. The memory map of the DSP external and internal RAM and ROM is given in the command files as shown in Figure 5.12. The 2 Kword internal RAM block is designated as two 1 Kword blocks. The 128 Kword Local Memory is 0 -wait state memory that is divided into 4 sections. The VECT section provides 512 words for interrupt vector tables. The LM0 section provides 32255 words for the DSP program and variables. LM1 provides a 32 Kword block of RAM for constants and arrays that are uploaded as well as those created and used during the program execution. LM2 provides a 64 Kword block of RAM for data storage and processing. The 0-wait state Near Global Memory is divided into two 64 Kword memory blocks. The 16 MWord DRAM is accessible only to the processor 0 DSP via the Global Register and the Shared Global Bus.

\section{Unpacking/Data Conversion}

Each DSP can record and store up to $512 \mathrm{~K}$ samples per recording. When the data is transferred via the local bus, the data is packed into a 16 bit format. When the DSP receives the data from the local bus via the on-board Ballistic chip, two 16-bit words are packed again into a 32-bit word before it is transferred to NGM RAM. A full 512K sample recording would require $256 \mathrm{KWords}$ of NGM memory simply for storage of the data. The current C4x modules are limited to $128 \mathrm{KWords}$ which must provide both the initial storage of the packed data as well as space to processing the data. Recordings larger than $64 \mathrm{KWords}$ would require extensive data 
I*SDOC

File : adc2dspc.md (modified vx8tim.cmd by Spectrum Signal Processing Inc. Copyright 1996) Contents : C40 linker map for TIM40 modules on the VX8 board(sites C-H). Code is placed in bank 0 SRAM. For external use. THIS MUST BE USED FOR BUILDING APPLICATION DESTINED FOR NODES C-H ON THE VX8 BOARD AND SHOULD BE COPIED AND MODIFIED TO MATCH THE TARGET PROCESSOR/TIM.

Original Author: VX8 Software

\$Date: 9/23/97 6:06p \$

\$Revision:: 4 $\$$

\$Archive:: /MAC/VX8_092.v10/dev/dsp/examples/vx8tim.cmd $\$$ Modified by: William L. Simkins, Jr., Eastern Shore Research Corp., 8/10/1999

Notes : (1) Object and library files to be linked are added by the make file, and are not listed here.

(2) When using the small (default) memory model, be sure that the ENTIRE .bss section fits within a single page. To satisfy this, .bss must be smaller than $64 \mathrm{~K}$ words and must not cross any $64 \mathrm{~K}$ boundaries.

(3) The loading mechanism used by the VX8 loads a small kernel onto processors specified in the host .sdf file. This COMM load kernel occupies 0x80000000 to $0 \times 800000 \mathrm{FF}$ inclusively. The User's $\mathrm{C} 4 \mathrm{x}$ application cannot place program or initialized data in this memory region labled "COM_KERNEL".

\begin{tabular}{|c|c|}
\hline$-\mathrm{cr}$ & $I^{*}$ link using c conventions - RAM model */ \\
\hline -stack $0 \times 200$ & $/ * 256$ stack \\
\hline -heap $0 \times 0$ & $/ *$ Specify heap size $* /$ \\
\hline$-\mathrm{x}$ & /* exhaustively search libs */ \\
\hline \multicolumn{2}{|l|}{$-1 v x 8 c 44$ ss. lib } \\
\hline \multicolumn{2}{|l|}{$-\operatorname{lrts} 40$. lib } \\
\hline \multicolumn{2}{|l|}{-lprts40.lib } \\
\hline \multicolumn{2}{|c|}{-lc:IsinectolCBlas123\Blas123.lib } \\
\hline \multicolumn{2}{|c|}{-lc:Isinectoldspvectrldspvectr.lib } \\
\hline
\end{tabular}




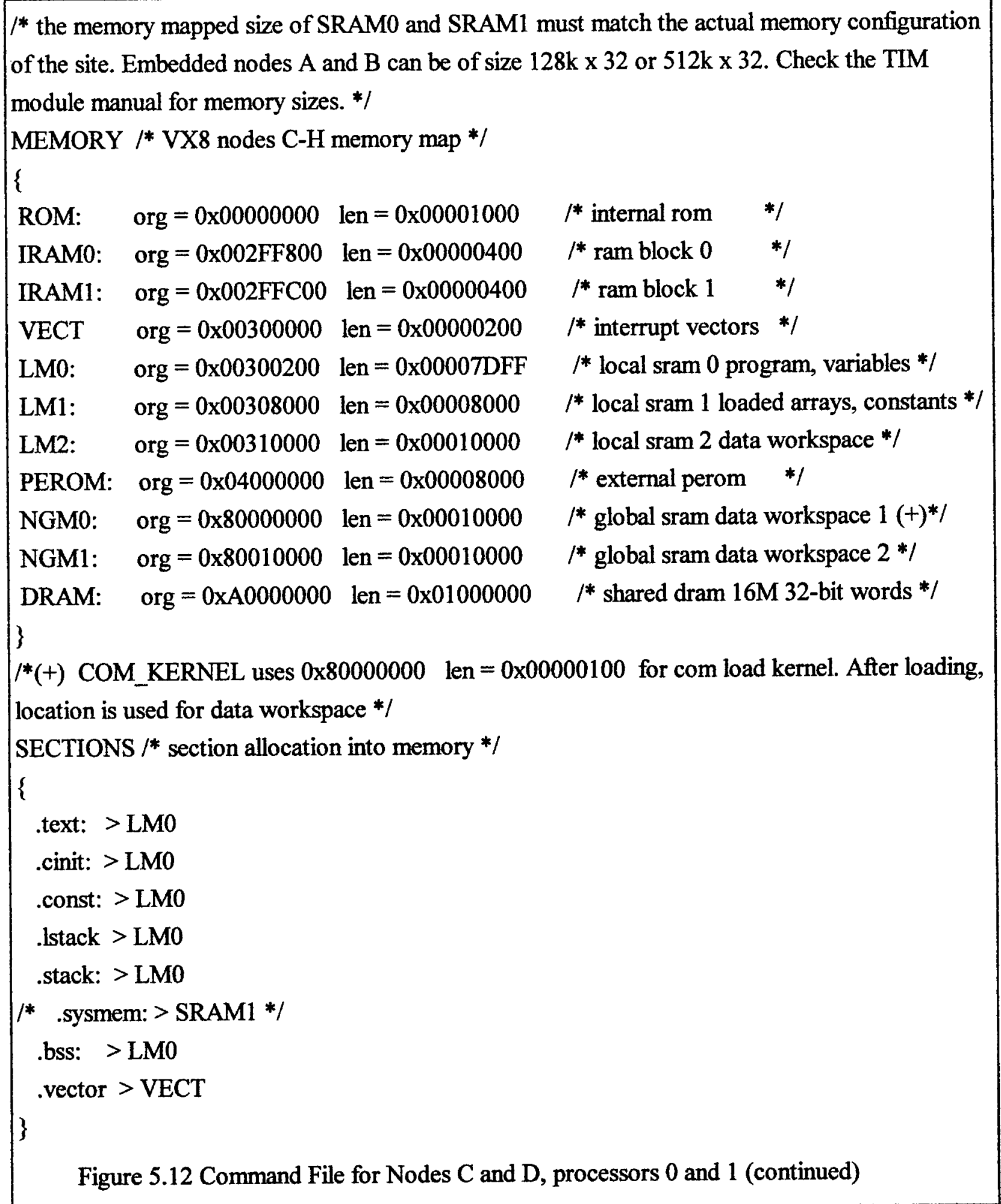


manipulation and reduce the processing thruput. Therefore, software was designed with a data recording limit of $64 \mathrm{~K}$ samples per recording with a maximum of 1024 samples per pulse. ${ }^{1}$

Before any processing is performed, a double unpacking operation has to be performed on the HP local bus data. First, a $\mathrm{C} 4 \mathrm{x}$ unpacking routine provided by Texas Instruments is used to unpack the HP local bus data into the original 16 bit packed words. Then, the packing provided by the HP 1429 digitizers are unpacked and rescaled to represent the true voltage integer values. These integer values are converted to $\mathrm{C} 4 \mathrm{x}$ single precision floating point formats. Any $\mathrm{DC}$ bias that was added by the digitizers is then estimated and subtracted from data.

\section{Digital I/Q}

The digital I/Q process is a frequency-domain technique using the Hilbert transform [3034]. The process transforms the input data on a pulse by pulse basis. The real data from each pulse is converted to a complex format and placed in internal RAM. A complex FFT of the data is followed by a vector multiply and decimation to create the analytic. This data is then stored in local memory awaiting transmission to the Node $\mathrm{C} 1$.

\section{Timing Profile}

Table 5.2 presents the estimated and measured timing for the processing provided by Node $\mathrm{C} 0$ and Node D0. The estimated values for the Sinectonalysis routines were provided in the vendor literature while other values were estimated from an analysis of the $\mathrm{C}$ or assembly routines. The example used the maximum $64 \mathrm{~K}$ samples to provide the maximum time bound. Node C0/D0 required .126 seconds to preprocess the data and perform Digital I/Q processing. With about .014 second to transfer the data to Node $\mathrm{C} 1$, the total time needed between Local bus transfers is .14 seconds.

All the estimated times were optimistic, but not overly so for the sample sizes used. Some of the differences between the estimated and measured times can be traced to the choice of memory and the additional wait states needed. Other differences are due to overhead in the routine. Finally, Code Composer provides a small error due to its need to flush the pipeline when it stops the processor for a measurement. The differences between the estimated and measured

\footnotetext{
${ }^{1}$ Current modules are now available with $512 \mathrm{Kword}$ memories for both Local and Global memories. These modules would allow the processing of 512K sample recordings. However, the Digital I/Q processing makes use of the internal RAM to increase the speed. Since the internal RAM is limited to $2 \mathrm{Kwords}$, the maximum number of samples per transform is still limited to 1024 samples.
} 


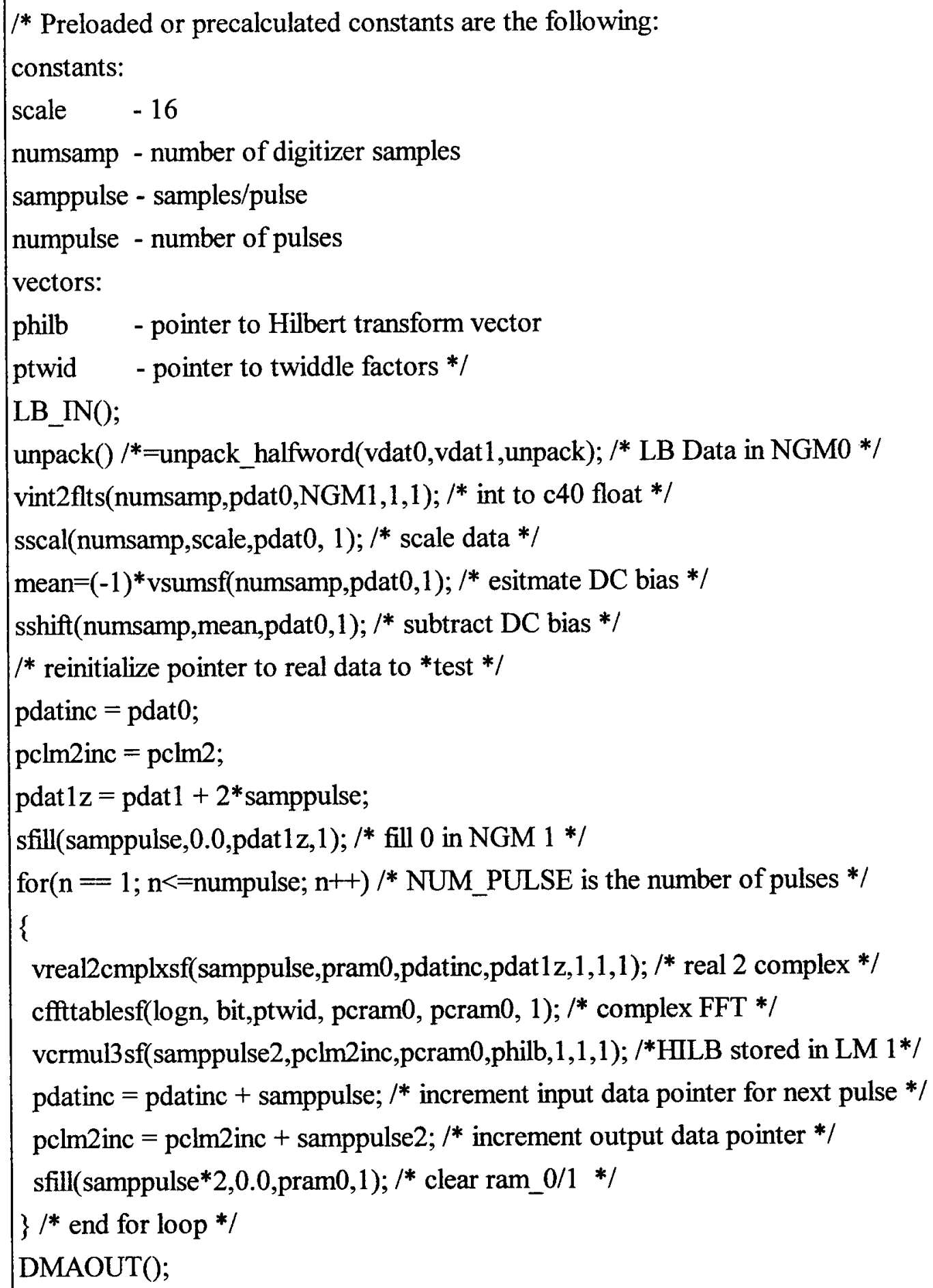

Figure 5.13 Preprocessing and Digital I/Q processing (Node C0/D0) 
times were typically within 3 percent and the estimates were useful in setting up the processing.

\subsubsection{Node C1 D1 - Range-Doppler Processing}

Processor 1 of the dual C44 module performs the channel compensation and the rangeDoppler processing. The memory map configuration used is identical to that provided for Processor 0 and given in Figure 5.12. Where reasonable, the internal RAM is used in computational intensive functions to increase speed.

\section{Channel Compensation}

Ideal receiver channels would have the same transfer function with flat gain and linear phase versus frequency within the passband and with identical attenuation in the stopband frequencies. Unfortunately, real channels not only have different transfer functions, but these transfer functions can change with time. The best approach to compensate for this problem is to measure the transfer function periodically and compute an inverse transfer function that will compensate for the non-ideal characteristics. [7-9]. During this effort, this compensation vector is computed in the host and uploaded to the host prior to processing. The compensation requires a complex vector multiply in the frequency domain.

\begin{tabular}{|c|c|c|c|c|c|c|}
\hline \multirow[t]{2}{*}{ Process } & \multirow{2}{*}{$\begin{array}{c}\text { Number of } \\
\text { Samples } \\
\text { (N) }\end{array}$} & \multicolumn{3}{|c|}{----- Estimated Values------------- } & \multicolumn{2}{|c|}{---- Measured Values --.- } \\
\hline & & Equation & Cycles & $\begin{array}{l}\text { Time } \\
\text { (usec) }\end{array}$ & Clock Cycles & $\begin{array}{r}\text { Time } \\
\text { (usec) } \\
\end{array}$ \\
\hline unpack & $65536 \mathrm{R}$ & $6 \mathrm{~N}$ & 196608 & 6489 & 201002 & 6700 \\
\hline data conversion & $65536 \mathrm{R}$ & $5 \mathrm{~N}$ & 327600 & 10920 & 400563 & 13352 \\
\hline setup/misc & N/A & 1024 & 1024 & 34 & 1324 & 44 \\
\hline \multicolumn{7}{|l|}{ Digital I/Q } \\
\hline * CFFT & $1024 \mathrm{C}$ & $4 N * 3.32 * \log (N)$ & 40936 & 1365 & 42139 & 1405 \\
\hline * hilbert xform & $512 \mathrm{C}$ & $4 \mathrm{~N}$ & 2048 & 68 & 3151 & 105 \\
\hline * misc & $1024 \mathrm{R}$ & $6 \mathrm{~N}$ & 6144 & 205 & 4299 & 143 \\
\hline * Total /pulse & $1024 \mathrm{R}$ & & 49128 & 1638 & 49589 & 1653 \\
\hline * Total /64 pulses & $32768 \mathrm{C}$ & & 3144192 & 104805 & 3173696 & 105789 \\
\hline \multicolumn{3}{|c|}{ Total Node C0 Processing } & 3668400 & 122268 & 3776585 & 125874 \\
\hline DMA to Node C1 & $32768 \mathrm{C}$ & $(2 * N * 4) * 30 / 20$ & 393216 & 13106 & 405000 & $13499^{*}$ \\
\hline \multicolumn{3}{|c|}{$\begin{array}{l}\text { Total btw HPLB Transfers } \\
\text { * estimate - see text }\end{array}$} & 4061616 & 135374 & $4181585^{*}$ & $139373 *$ \\
\hline
\end{tabular}




\section{Matched Filtering}

After compensation, the data is multiplied by a weighting function to reduce the range sidelobe prior to matched filtering. During this effort, a Hamming weight is used to provide a nominal $-40 \mathrm{~dB}$ weighting. The weighted signal is then matched filtered prior to inverse FFT back to the time domain.

For modulated signals used in pulse compression, many of the time domain samples near the end of the window represent data that is not fully processed. For a waveform that is pw samples in length, an $\mathrm{n}$ sample window will only contain $\mathrm{n}$-pw+1 fully compressed outputs. The example used in this effort was the 40 usec pulse used in many of the earlier measurements [8,9] and used as a tracking waveform by RL's S-Band monostatic tracking radar. This waveform is 800 samples long after digitization and 400 samples long after Digital I/Q processing. For the 512 sample window (1024 samples before decimation), this leaves only $512-400+1=113$ samples that are fully processed after matched filtering. Therefore, only 113 samples need to be passed to further processing.

\section{MTI}

Moving Target Indicator (MTI) processing provides a notch filter and is used to reduce the amplitude of strong land clutter. In regions of weak clutter returns, MTI is not necessary and can be bypassed. However, much of the current interest involves illuminating land clutter and MTI processing is usually a necessity. The MTI weights are implemented as a complex weight to allow the steering of the notch and tuning of the bandpass. This capability allows the bistatic system to be used with an airborne illuminator where the land clutter return would have a non-zero mean Doppler component. Preparation of the system for such use would require concurrent or precalculation of the MTI weighting to steer the notch to the clutter mean frequency.

\section{Doppler Filtering}

The Discrete Fourier Transform processing provides Doppler filters for estimating mean target velocities and resolving moving targets in the same range cell. This DFT also provides additional attenuation to clutter. The DFT implementation makes use of the fast FFT routines provided by Sinectoanalysis, Inc. First, the returns from consecutive pulses from a single range cell are weighted to reduce the Doppler sidelobes. Then the Doppler filters are created using 


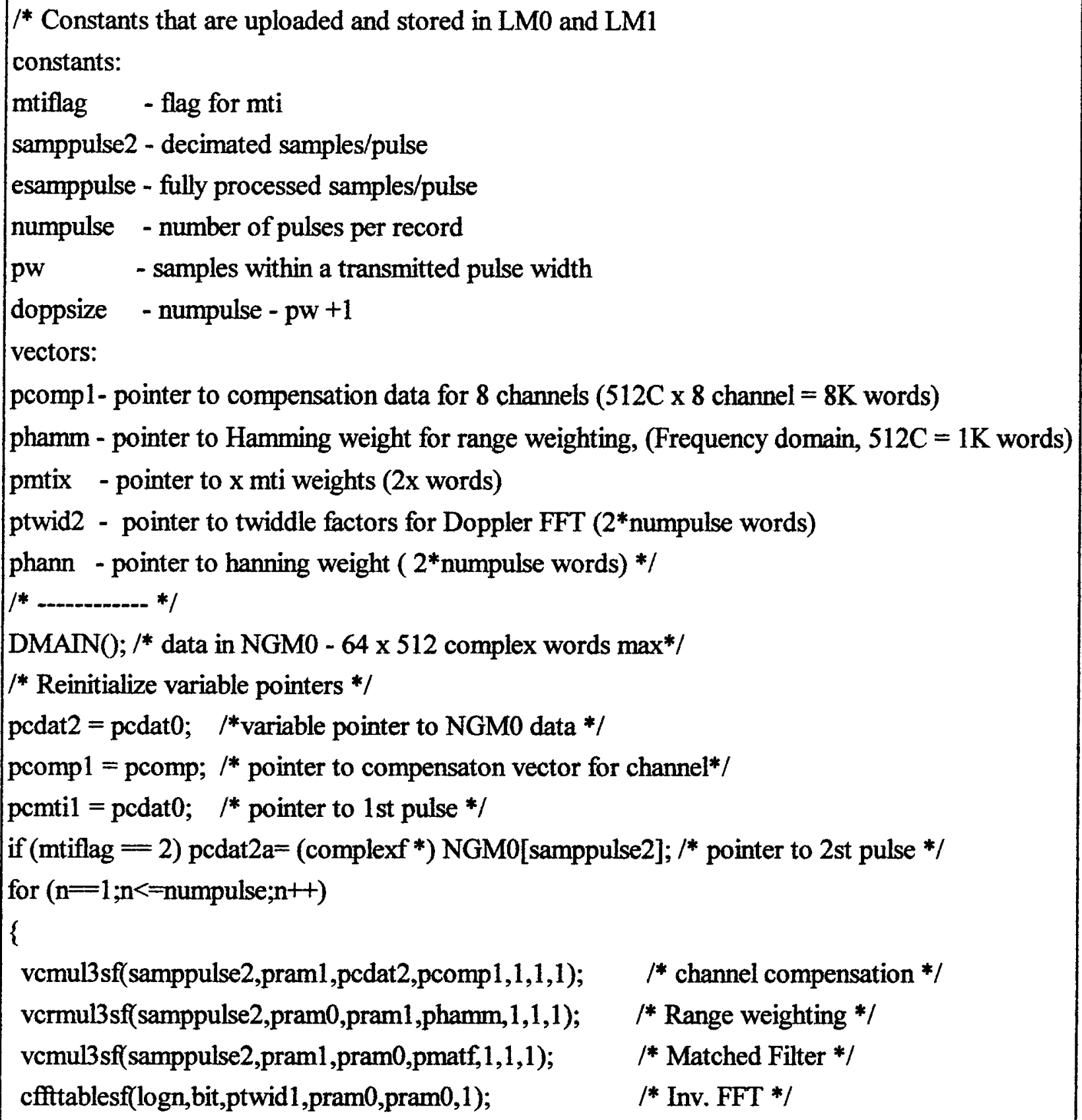

Figure 5.14 Channel Compensation and Range-Doppler Processing 


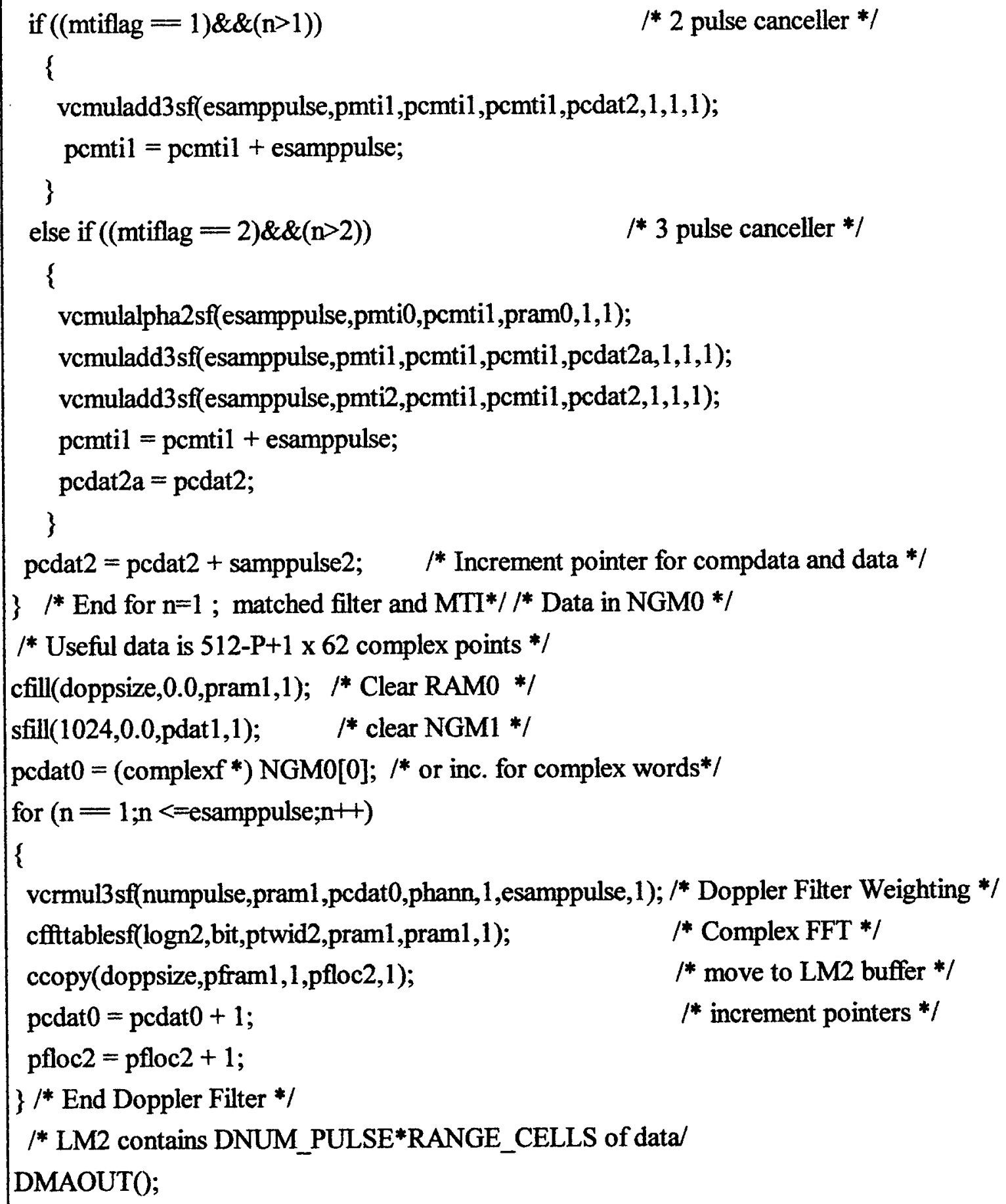

Figure 5.14 Channel Compensation and Range-Doppler Processing (Continued) 
a complex FFT routine. After the DFT process, the outputs represent a complex range-Doppler vector that is transferred to Node $B$ for antenna beamforming processing.

\section{Timing Profile}

Table 5.3 presents the estimated and measured timing for the processing provided by Node $\mathrm{C} 1$ and Node D1. The estimated values use the equations provided by Sinectoanalysis, Inc. while the other estimates were obtained from an analysis of the $\mathrm{C}$ or assembly routines. The example used the maximum $64 \mathrm{~K}$ recording as used for Node $\mathrm{C} 0$. Node $\mathrm{C} 1$ requires less than 79 milliseconds to perform channel compensation, matched filtering, MTI and Doppler Filtering. Since the pulse width is a large percentage of the number of samples digitized per pulse (400 of the 512 decimated samples), only $(512-400+1) * 64=7232$ samples need to be transferred to Node $\mathrm{B}$ for antenna beamforming.

Over $50 \mathrm{msec}$ (1500150 cycles) of time are available for additional processing or for changes in processing parameters. For example, if the bistatic system is used in conjunction with a high power transmitter system with a shorter pulse compression waveform, the extra time still allows full processing. For example, with a 6 microsecond pulse (60 decimated samples/pulse width), the number of fully processed samples is $512-60+1=453$ samples or 7.5 range cells. The additional number of cycles required for Doppler processing is $975,309-243289=732020$ cycles or an additional $24.4 \mathrm{msec}$. The number of samples available to transfer will be $64 * 453=28992$ samples requiring an additional 347904 cycles or $11.6 \mathrm{msec}$ to transfer. The total additional time of $35 \mathrm{msec}$ is well within the additional time available between DMA transfers from Node C0.

\subsection{Node B - Digital Antenna Beamforming}

Node C0 and Node D0 transfer range-Doppler data to the Local Memory buffer LM2 of node $\mathrm{B}$ via DMA transfers thru dedicated Comm ports. Node $\mathrm{C} 1 \mathrm{Comm}$ port 2 is connected to Node B's Comm port 4. Node D1 Comm port 1 is connected to Node B's Comm port 5. Figure 5.11 provides an indication of the relative timing of these two transfers. The data transferred is a reduced set representing the decimated fully processed outputs. For the $64 \mathrm{~K}$ example, this represents 64 x $113=7232$ samples per channel.

\section{Beamforming}

Beamforming is performed by multiplying the new data by the channel weight for each beam and adding these results to the weighted values from earlier channels. The data is stored in the Near Global Memory NGM. The number of range-Doppler-beam samples is limited by the size of the NGM in Node B. This limit is currently $128 \mathrm{KWords}$ or 65635 complex samples. For the 
Table 5.3 Timing Profile for Node C1/D1 Processing per 64K record

Process Number of ----- Estimated Values------------

---- Measured Values ----

Samples Equation Cycles Time Cycles Time

(N)

(usec) (usec)

Preparation

Processing/pulse

Range Matched Filter

* Compensation $\quad 512 \mathrm{C} \quad 7 \mathrm{~N}$

$\begin{array}{lll}3584 & 120 & 4690\end{array}$

156

* Range Weighting $512 \mathrm{C} \quad \mathrm{N}$

$3072 \quad 103$

3154

105

* Matched Filter

$512 \mathrm{C} 6 \mathrm{~N}$

$3072 \quad 103$

3154

105

* ICFFT

$512 \mathrm{C} \quad 4 \mathrm{~N} * 3.32 * \log (\mathrm{N})$

18421

$614 \quad 19149$

638

* 3PMTI

$113 \mathrm{C} \quad 36 \mathrm{~N}$

$4068 \quad 136$

2308

77

* Misc

* Total/Pulse

* Total/64 Pulses

$\begin{array}{llll}32217 & 1074 & 32455 & 1082\end{array}$

Preparation

$2061888 \quad 68722$

2077120

69169

224

75

DFT/range cell

* Weighting

$62 \mathrm{C} \quad 4 \mathrm{~N}$

248

397

13

* CFFT

$64 \mathrm{C}$

$4 N * 3.32 * \log (N)$

$1535 \quad 51$

1840

* Misc

* Total/Range Cell

$2153 \quad 72 \quad 2631$

88

* Total/113 Range Cells $243289 \quad 8109 \quad 297303$

9909

Total Node C Processing

$2305177 \quad 76832 \quad 2376696$

79215

DMA to Node $\mathrm{Cl} \quad 7232 \mathrm{C} \quad(\mathrm{N} * 4) * 30 / 20$

$86784 \quad 2893 \quad 101250$

$3375^{*}$

Total processing time

$\begin{array}{lll}2391961 & 79725 & 2472991\end{array}$

82363*

Extra time available for additional processing

166965555649

1708594*

$57010^{*}$

* estimate - see text 


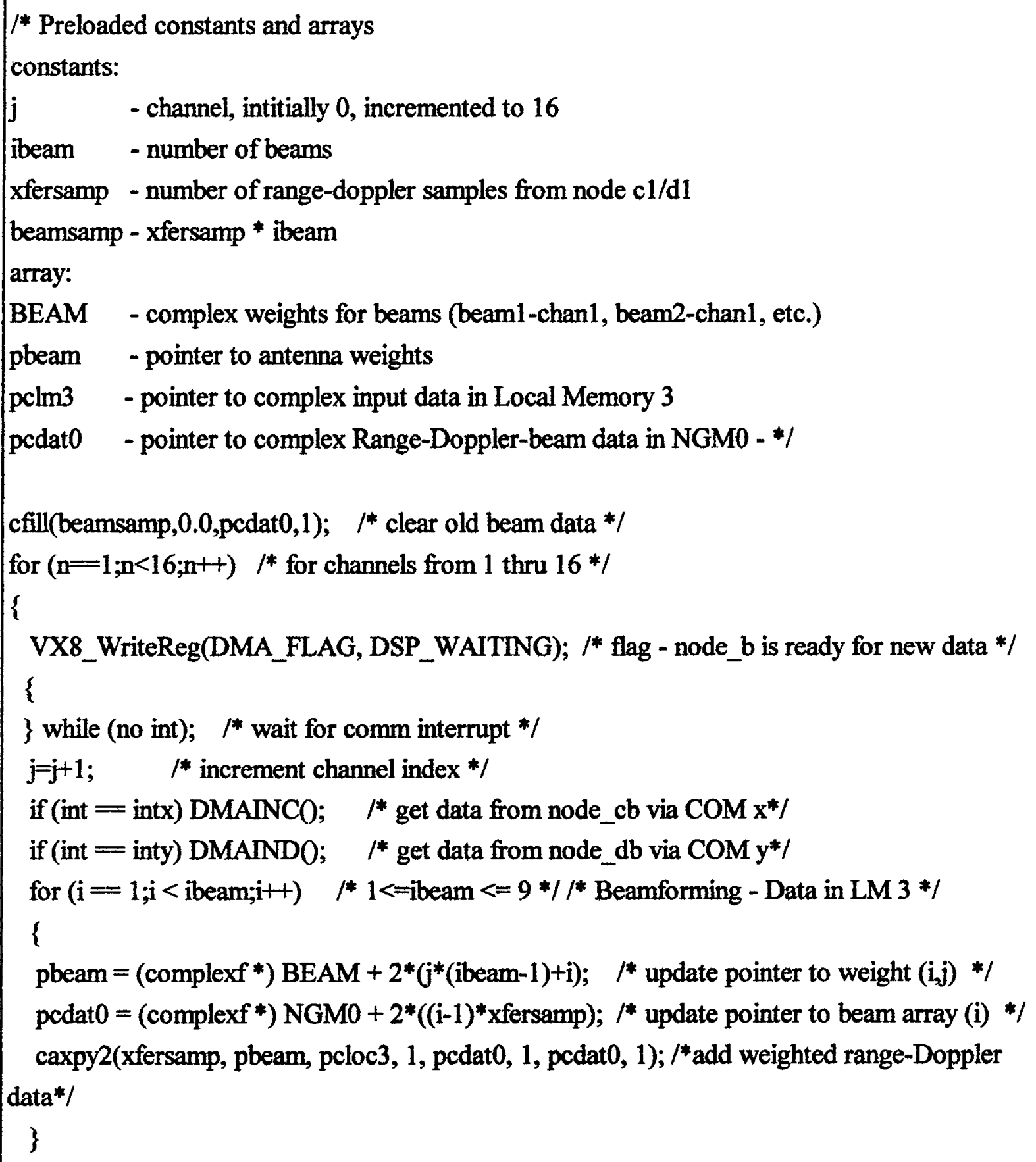

Figure 5.15 Beamforming Processing (Node B) 
\} $/ *$ for $(\mathrm{n}==1 ; \mathrm{n}<16 ; \mathrm{n}++) * /$

vflt2ieees(beamsamp*2, pfdat 0, pfdat $0,1,1) ; \quad / *$ convert to IEEE */

VX8_Lock(DRAM0); /* Lock Global Shared Bus */

VX8_FastTransfer(DRAM0, NGM(0),status); $/ *$ transfer data to DRAM */

VX8_UnLock ()$; \quad / *$ Unlock Global Shared Bus */

VX8_WriteReg(HOST_FLAG, DSP_DONE); /* Tell Node A/host - DRAM data is ready */

Figure 5.15 Beamforming and Data Transfer (Node B) (continued)

Table 5.4 Timing Profile for Node B Processing per 64K record

Process Number of -.--- Estimated Values----------- $\quad$---- Measured Values --Samples Equation Cycles Time Clock Cycles Time

(N) (usec) (usec)

\section{Beamforming}

* Misc

$30 \quad 1$

30

* Channel Wt.

$\& \mathrm{add} /$ beam

$7232 \mathrm{C} \quad 2 * 6 \mathrm{~N}$

$86784 \quad 2893$

115800

3860

* Total/Beam

$86814 \quad 2894$

115830

3861

* Total/9 Beams

$781326 \quad 26042$

1042470

34746

* Nodes C1+D1

$1562652 \quad 52084$

2084940

69492

Extra time available

between $\mathrm{C} 1$ transfers

2498964

83290

1976676

65883

FLT->IEEE $\quad 65088 \mathrm{C} \quad 2 * 4 \mathrm{~N}$

$520704 \quad 17355$

520755

17357

/Transfer to DRAM $65088 \mathrm{C} \quad 2 * 7 \mathrm{~N}$

$911232 \quad 30371$

Flag NodeA/host

76

$1000000^{*}$

33330*

Misc/Setup

100

3

76

100

* estimate - see text 
example used, this represents 9 simple beams. The data for each beam is stored in range order for each Doppler filter.

For more complex beams such as an adaptive beamforming process, up to two beams can be stored within the allocated $32 \mathrm{KWords}$ for LM1 where a different beam weight would be stored for each range-Doppler cell.

\section{Timing Profile}

Table 5.4 presents the estimated and measured timing for the processing provided by Node B. The example used the same $64 \mathrm{~K}$ recording used for the other node. However, due to decimation and the long pulse width, only 7232 fully processed complex samples are contained in each transfer from Nodes $\mathrm{C} 1$ and $\mathrm{D} 1$. The measured time needed to process two transfers, one transfer from Node $\mathrm{C} 1$ and one from Node D1, is about 69.5 milliseconds out of the 135 millisecond processing period between consecutive transfers from $\mathrm{C} 1$ (Figure 5.11). Roughly half of the processor's capability is available for other real-time processing.

The beamforming process creates nine beams and stores the range-Doppler-beam data in its Near Global Memory. After the data from the last channel has been received from Node D1 and integrated into the nine beams, the data is converted from the $\mathrm{C} 4 \mathrm{x}$ floating point format to the IEEE floating point format and transferred to the DRAM to await transfer to the host. Some undescribed conflict with Code Composer created delays over 1 second during attempts to profile this transfer and prevented using Code Composer to accurately measure this time. An article in the TI Designer's notebook [24] seems to offer an explanation for this problem. Consequently, the transfer time to DRAM given in Table 5.4 is an estimate.

\subsubsection{Node A - Host Interface and Interrupt Servicing}

In this effort, Node $\mathrm{A}$ is used primarily as a host-C4x interface, a boot loader for the VX8 board and to service interrupts from the VXIbus. Node $A$ has several unique capabilities that prevented its consideration as a dedicated pipeline processor. First, Node $\mathrm{A}$ has the unique capability of suspending the HP Local Bus DMA transfers, allowing Node A to access the Global Shared or DRAM Shared Bus as needed. Second, Node A's local bus has a A16 Control/Status register support to provide VXIbus A16 support and an Interrupt Route Matrix control register interface. This allows Node A to receive interrupts and to have the complete control of the VX8 Shared Buses needed to service them. The above functions take only a small amount of Node A's processing capability. Therefore, Node $\mathrm{A}$ is available to perform auxiliary processing as needed. 


\subsubsection{Data Transfer and System Thruput}

The original intent of the effort was to integrate the DSP software with the existing bistatic control software. However, during the interface software development, two major incompatibilities were discovered between the VX8 system and the existing bistatic radar. First, the VX8 software was incompatible with the HP 1489C/E1482B MXI interface in the existing system. Second, the VX8 host software was incompatible with the Borland $\mathrm{C}++$ software development system used to create the existing system software. Other difficulties are discussed in a later section titled Lessons Learned.

In June 1999, AFRL replaced the HP MXI interface with a National MXI-2 interface that was compatible with the VX8 host software. With respect to profiling the system thruput, the incompatibility between the VX8 software and Borland prevented the development of the host VX8 software routines and the integration the VX8 software into the existing system control software. This prevented any tests of the Local Bus and the VXIbus data transfer rates to and from the VX8 board. After following many suggestions from Borland and Spectrum on how the VX8 libraries might be used with Borland without success, a copy of Microsoft Visual C was purchased to complete the DSP tests. Redoing the existing bistatic control software was beyond the scope of the current effort. Instead, small programs were created to test the VXIbus communication between the host and the VX8 board, to test the VXIbus and Local Bus data transfer routines provided by Spectrum and to obtain estimates of data transfer times and thruput rate. Estimates of these transfer times were then used with the other measurements to estimate the total processing time from digitization to storage or display of the data on the host.

\section{VX8 to host Transfer}

A small program was created to test the transfer of data from the VX8 DRAM to the host computer. The test transferred 65088 complex words (130176 32-bit words) to memory on the host and store the data on a file. The transfer took less than $.5 \mathrm{sec}$ which corresponds close to the 2 MBytes/sec data rate expected.

\section{HP-Local Bus transfer}

A host small program was created to test the data transfer rate from the digitizers to the VX8 board. The program initialized the digitizers to collect a $64 \mathrm{~K}$ sample and then sequentially transfer the data starting with the first digitizer. A test program based on one of Spectrum's example programs was created to receive the data. The program was to simply transfer the data 
from first digitizer to the NGM of a C44 module, then immediately overwrite the data by the next transfer. The purpose was to estimate the total time needed to transfer the 16 channel data using the host controller. An attempt was made to use Code Composer to measure the transfer time. However, some conflict similar to the DRAM transfer problem mentioned earlier prevented an accurate measurement. Observations indicated that the time required to transfer the data in all 16 channels of data took about 3 seconds. However, much of this time was caused by the overhead in host processing and VXIbus delays in resetting and restarting the VXI modules required by the local bus protocol. There was also a problem in resetting and restarting the $\mathrm{C} 4 \mathrm{x}$ module running the modified example program. Spectrum has recently provided newer versions of the VX8 software that have re-addressed the HP Local routines [25].

\section{System Timing and Thruput}

Figure 5.11 presents a functional description of the timing of the parallel pipeline system. Much of the processing is being performed in parallel, reducing the total processing time. Using the measurements and estimates described earlier, this figure can be used to estimate the total time needed to process the data. Table 5.5 adds the estimated and measured times and shows that the complete process requires about 1.6 seconds. This estimate does not include the overhead times associated with the Win NT host software which can easily increase the total time to 4.5 seconds.

The thruput of the example is a less impressive $130 \mathrm{KBytes}$ per 1.6 seconds. One reason for this low thruput rate is the number of bytes discarded. While 2 MBytes of data was transferred from the digitizers, the large reduction in samples is due to the decimation in the digital $I / Q$ process and the large number of samples in the pulse width. A similar analysis using a shorter pulse such a 6 microseconds would provide a thruput of over $500 \mathrm{KBytes}$ per second. Second, the SRAM of each $\mathrm{C} 4 \mathrm{x}$ module is limited to $128 \mathrm{KWords}$. This limits the size of the data that can be transferred and processed simultaneously. It also requires the use of the digitizers as memory storage until all the data transfers can be completed. This forces the bistatic system to operate in a batch mode.

The processing time provides the bistatic system with a real-time adjunct capability that can be demonstrated with many existing monostatic systems with scan rates of 5 seconds or more. This real-time capability can be used in demonstrating bistatic target detection or making bistatic clutter and attenuation measurements within a small range-azimuth sector. The multiple-pulse burst recordings provide the samples needed to resolve the attenuation signal from the clutter while multiple burst recordings, taken at a 2-3 second rate, should provide uncorrelated samples of the changes in the attenuation signal and clutter returns. 
Table 5.5 Total System Timing per 64K Record (See Figure 5.11)

\begin{tabular}{|rr} 
Process & $\begin{array}{r}\text { Time } \\
\text { (usec) }\end{array}$ \\
\hline Time difference between transfer of channel 1 and channel 2(1) & 3000 \\
HP Local Bus Transfer/channel $(65536 * 2$ bytes/40MBytes/usec) $(2)$ & 3280 \\
Digital I/Q/channel+xfer to node C1 & 139373 \\
Total per channel & 141558 \\
8 channels & 1132464 \\
& \\
Range-Doppler Processing-last channel & 82363 \\
Beamforming - last channel & 69492 \\
Floating Point Conversion and transfer to DRAM & 50687 \\
Transfer to Host $(65088 * 2 * 4=520704 \text { bytes @ 2Mbytes/sec) })^{(2)}$ & 250000 \\
Total time per $64 \mathrm{~K}$ collection & 1588006
\end{tabular}

(1) estimated time to reset $\mathrm{C} 4 \mathrm{x}$ and other VXI modules for next transfer. Does not include overhead caused by host software.

(2) estimated using advertised values 


\subsection{LESSONS LEARNED}

This effort involved trying to integrate a newly developed product into an existing system. It was recognized from the start that new products may have bugs or characteristics that are not completely defined for all situations. Consequently, the 10 month contract was scheduled to begin with a six month software development and debugging period from Mid-December 1997 thru Mid-May 1998 and a four month testing period from Mid-May 1998 thru Mid-September 1998. One of AFRL's obligations in this contract was to purchase the VXI-based DSP board and associated hardware and software and to provide access to the existing bistatic system, the DSP board and the associated development hardware and software at the beginning of the contract. However, several of the difficulties occurred that were not foreseen and had a significant impact on the effort. This chapter lists some of the difficulties incurred during the effort and the lessons learned during the process.

\section{A. Delays and Lack of Timely Information}

Some of the most significant unforeseen problems were the delays incurred after the proposed schedule was accepted. These delays include the lack of timely information on the MXI interface and $\mathrm{C}$ compiler requirements and a delay in providing the JTAG emulator.

\section{LeMans specifications regarding MXI interface requirements:}

The earlier information provided by Spectrum was that shown in Appendix B-1. The information indicated that the system would be usable in a Windows environment (Windows 95 and WinNT) using VISA resource managers. Initial discussions with Spectrum indicated that the VX8 would be sold thru Hewlett-Packard (HP), the vendor of the existing MXI interface module. None of the literature or discussions with HP nor Spectrum, prior to the purchase of the system, indicated that the existing MXI interface was incompatible with the LeMans VX8 support software and drivers.

In December 1997, Spectrum originally delivered the LeMans VX8 board with UNIX software, not the WinNT software ordered. The accompanying manuals presented some discussion of the VISA implementation, but focused mainly on the UNIX/SICL software. The description of the host-VXI interface did not require the use of a MXI-2 interface instead of the Hewlett Packard MXI interface existing in the system. The revised versions of the VISA interface documents received in early 1998 did admit that the software was developed using a MXI2 interface and it took several hours of effort to determine that the drivers were not compatible with the HP MXI interface. If this information was provided prior to purchase, AFRL would have been 
given the opportunity to allocate funds for the purchase of a MXI2 board in 1997 for use in this effort in 1998.

\section{Delay in receiving the JTAG interface}

Initially, the development and testing of the DSP software were expected to dominate software development time. Therefore, the effort was to begin with a six month development period before taking the system to the Newport site in May 1998. The DSP software development system chosen by AFRL was Code ComposerTM, a system developed by GO DSP. However, the JTAG emulator required by Code Composer was not delivered with the Code Composer software at the start of the effort. It was finally delivered in May 1998 after the system was moved to the Newport site. The impact of this delay was to eliminate the 6 month DSP software development and debugging period proposed in the original schedule and to make the development and testing of the DSP software within the remaining four months of the original contract schedule unlikely.

\section{Delay in receiving source code and $\mathrm{C}$ compiler requirement information}

The existing bistatic system software was developed using Borland $\mathrm{C}++4.5$. The original information on the VX8 module did not indicate a preference for a particular version of $C$. When purchase information was obtained by AFRL, there was a reference that the modules were developed with Microsoft Visual C 5.0. However, Spectrum indicated that the VXI Instrument Driver was written in $\mathrm{C}$ and that the source code would be provided to allow optimization with a user's system. Unfortunately, the source code for one module, the System Definition Module, was not provided. This library, sdf_32.lib, was a static library that was not readable by Borland. Consequently, a Borland-compatible version of the VX8 Instrument Driver could not be created during the effort.

Several standard procedures were attempted to use the Microsoft-based libraries without success. These procedures include the use of IMPDEF and IMPLIB on the VX8 Driver DLL and explicit linking. In August 1999, Spectrum provided the password for a newer version of the VX8 software that could be downloaded from the Spectrum web site. Spectrum also suggested that explicit linking might be used successfully with this newer version.

None of the Spectrum literature received before the purchase of the VX8 board referenced an incompatibility with Borland. After the purchase, the statement that no Borland support was currently available was found in only one of the tardy VISA manuals [27, p31]. If this information was provided in 1997, AFRL would have had time to consider recreating all the existing bistatic control software in a Microsoft Visual C environment. 
The lesson to be learned is that purchasers of current equipment and software must get vendors to commit on all the interface and compatibility requirements before purchasing the equipment.

\section{B. Code Composer ${ }^{\mathrm{TM}}$}

Code Composer ${ }^{\mathrm{TM}}$ is a comprehensive Integrated Development Environment (IDE) developed by GO DSP, a Texas Instruments Company. Once one becomes familiar with Code Composer, it was pleasure to use. Few problems were encountered when programming within one C4x DSP module. When combined with the DSP routines from Sinectonalysis, the environment made DSP programming and testing easy and quick.

However, some problems were encountered using Code Composer for testing and profiling data transfers using the Global Shared Bus to DRAM or the NGM of another DSP, using DMA and the COMM ports or using the HP Local Bus interface. The debugger seems to hang and profiling gives incorrect timing values. This problem prevented getting measured times for the DMA transfers between the DSP modules, the HP Local Bus transfers and the data transfers from Node B to the DRAM. A designer note from TI [27] indicates some of the issues that might be creating the problem. 


\subsection{SUMMARY AND RECOMMENDATIONS}

This report presents the results of two tasks performed during this effort. First, measurements were performed on the attenuation of foliage and the impact of screening on the detection of a small moving vehicle. The second task developed some near real-time radar signal processing software for a DSP module that was recently added to the bistatic system.

The foliage attenuation measurements included one-way and two-way measurements of attenuation at various locations along a road that offered various depths of foliage screening from a few feet to over 100 feet. The one-way measurements of the direct path through the forest indicated attenuation values at short depths from 10 to 20 feet could be approximated by a constant attenuation rate of $1 \mathrm{~dB} / \mathrm{ft}$ for dry vegetation and $2 \mathrm{~dB} / \mathrm{ft}$ for wet vegetation. Measured values range from $8 \mathrm{~dB}$ at 9 foot depth to $15 \mathrm{~dB}$ attenuation at a 17 foot depth. However, at positions along the road representing depths of 33 feet and 118 feet, little additional attenuation was observed. It was noted that energy from angles other than the direct path was comparable to the energy measured from the direct path. It was suggested that indirect illumination from multipath could provide the dominate illuminating energy in regions where the attenuation of the direct path energy was high. It is suggested that this is geometry related and that higher multipath energy would be obtained in screened regions that had an unobstructed access to directly illuminated vegetation or structures.

The screened vehicle measurements along the same road supported the suggestion that multipath energy could increase the detection of moving targets along screened roads above that indicated by the direct path attenuation only. A small vehicle target was detected along a road even when the direct signal was screened by over 100 feet of foliage.

The LeMans VX8 Digital Signal Processor (DSP) module was physically integrated in the bistatic VXI system. The VX8 system contains 2 embedded $60 \mathrm{MHz}$ TMS320C40 DSPs and two of Spectrum's MDC44ST twin TMS320C44 modules providing up to 360 MFLOPS of peak processing power. The VX8 system also features a Local Bus interface compatible with HP Local Bus protocol and host interface software compatible with Win NT and VISA.

The DSP software development focused on replacing the radar signal processing that had been performed off-line [8,9]. These routines included digital $\mathrm{V} / \mathrm{Q}$ demodulation, channel compensation, range matched filtering, 3PMTI, DFT and antenna beamforming. A parallel pipeline approach was used to provide a near real-time processing capability that could be used in 
field experiments and demonstrations. The DSP software was designed to process 64 pulse bursts for up to 1024 range samples and nine azimuth beams using uncoded or pulse compressed waveforms. This corresponds to fully processed outputs for up to a $4 \mathrm{~nm} \times 60$ degree sector in less than 2 seconds.

Several recommendations for the upgrade and use of the bistatic system were developed during the effort. First, the VX8 software was incompatible with the Borland $\mathrm{C}++$ development system used to develop the existing system control software, preventing integration of the DSP software. It is recommended that this integration be completed. Spectrum recently provided an upgraded version of the VX8 software and suggested that this software might be usable with Borland [25, 29]. An alternate approach is to rewrite the existing system software in the Microsoft Visual $\mathrm{C}$ environment used to develop the VX8 software. This development should also include the creation of $\mathrm{X}-\mathrm{Y}$, range-Doppler and range-angle displays to allow the rapid display of the DSP outputs. Also, the integration of AFRL's FAA radar display interface would augment the performance of demonstrations and experiments.

It is also recommended that the VX8 subsystem be upgraded with additional memory and DSP modules. One of the current limitations is size of the Near Global Memory in each processor. This is the memory used by the HP Local Bus interface and its size limits the amount of data that can be downloaded from the digitizers in a single transfer. The current memory limits the transfer size to about $25 \%$ of the digitizer's memory. SRAM memory upgrades from the current 1 MByte/ processor to $4 \mathrm{MBytes} /$ processor can increase the processing size to match the digitizer memory and increase the processing sector size up to a $16 \mathrm{~nm} \times 60$ degree sector while still providing near real-time processing at less than an 8 second rate.

An additional 2 modules will extend the capability of the current software to process a 32 element array system while maintaining a 2 second processing rate. If used in the existing 16 element array, the additional modules offer a tradeoff of providing additional sector coverage or reducing the processing time.

One of the issues in the use of the non-real-time measurements performed during the last three years is the time required to perform, store and process a single set of measurements. One 128 pulse recording required over 15 minutes to off load into storage. Processing off-line using MATLAB required from 15 minutes for one range-Doppler-beam profile to over an hour for the processing needed for a complete set of range-azimuth displays of each Doppler filter. These long recording and processing times limited the number of measurements that could be taken and 
processed in a day. One of the advantages of near real-time processing is the ability to obtain and process the number of measurements needed to provide statistically valid conclusions on the issue being measured.

It is recommended that the existing system be used to address some of the issues in the foliage penetration problem. One recommended set of measurements is the attenuation of foliage at $\mathrm{S}$-band which provide the mean and fluctuation statistics of the attenuation as a function of foliage depth, wind conditions and leave moisture. Simultaneously, the measurements could also obtain the mean and fluctuating statistics of the clutter return from the foliage. Another set of recommended measurements was the mean and fluctuation of the radar cross section of screened military targets under the same conditions as given for the attenuation measurements. The recent additional of a UHF capability would provide the opportunity to perform the same measurements at UHF, allowing a comparison as a function of frequency. 


\section{BIBLIOGRAPHY}

[1] Wang, H. and Cai, L., "On Adaptive Spatial-Temporal Processing or Airborne Surveillance Radar Systems", IEEE Transctions on Aerospace and Electronic Systems, AES-30, 3 (July 1994), 660-670

[2] Barbarossa, S and Farina, A. "Space-Time-Frequency Processing of Synthetic aperture Radar Signals,", IEEE Transactions on Aerospace and Electronic Systems, AES-30, 2 (April 1994), 341-358

[3] Fante, R. L., "Cancellation of Specular and Diffuse Jammer Multipath Using a hbrid Adaptive Array", IEEE Transactions on Aerospace and Electronic Systems, AES-27, 5 (September 1991), 823-836

[4] Morgan, D. R. and Aridgides, A., "Adaptive Sidelobe cancellation of Wide-Band Multipath Interference", IEEE Transactions Antennas and Propagation, AP-33, August 1995), 908-917

[5] Tang, C. E. T., Liu, K. J. R., and Tretter, S. A., "Optimal Weight Extraction for Adaptive Beamforming Using Systolic Arrays", IEEE Transactions on Aerospace and Electronic Systems, AES-30, 2 (April 1994), 367-384

[6] Simkins, W. L., Multichannel Receiver Characterization for Adaptive Array Applications, Phase 1: Characterization of the Bistatic Receiver Testbed, Dec. 1995

[7] Simkins, W. L., Multichannel Receiver Characterization for Adaptive Array Applications, Phase 2: Algorithms for Calibration of the Bistatic Radar Testbed, April, 1997

[8] Simkins, W. L., Multichannel Receiver Characterization for Adaptive Array Applications, Phase 3: Assessment of the Bistatic Receiver Testbed as a Measurement Tool for Clutter Phenomenology, April, 1998

[9] Simkins, W. L., Multichannel Receiver Characterization for Adaptive Array Applications, Phase 4: Calibration Procedures/Tests. AFRL-SN-RS-TR-1998-228, January 1999

[10] Test and Measurement Catalog, Test and Measurement Sector, Hewlett-Packard, Santa Clara, CA, 1993 
[11] Skolnik, M. I., Introduction to Radar Systems, McGraw-Hill Book Company, New York, 1962

[12] Scheer, J. A. and Kurtz, J. L. (editors), Coherent Radar Performance Estimation, Artech House, Boston, MA, 1993

[13] HP E1429A/B, $20 \mathrm{MSa} / \mathrm{s}$ 2-Channel Digitizer, Users Manual, Hewlett Packard June, 1993

[14] Ulaby, Fawwaz T. et al, " Measuring the Propagation Properties of a Forest Canopy Using a Polarimetric Scatterometer", AP-38, 2 (February 1990), 251-258

[15] Fleischman, J.G., et al, " Part 1: Foilage Attenuation and Backscatter Analysis of SAR Imagery", AES-32, 1 (January 1996), 135-144

[16] Raney, R. Keith, "Synthetic Aperture Imaging Radar and Moving Targets", AES-7, 3 (May 1971), pp 499-505

[17] Willis, Nicolas J., Bistatic Radar, Technology Service Corporation, Artech House, Norwood, MA, 1991

[18] Real Property Tax Map, Town of Newport, Herkimer County, Map \# 93.004, Oct. 30, 1978

* [19] Simkins, W. L., Clutter Analysis for ADI, RL-TR-95-31, March 1995

[20] LeMans VX8 Carrier Board Technical Reference, Spectrum Signal Processing, Document number 500-00329, Revision 2.00, November, 1997

[21] TIM-40 TMS320C4x Module Specificaiton Version 1.01, Texas Instruments

[22] MDC44ST Super SRAM Dual C44 User Guide, Spectrum Signal Processing, Document Nnumber 500-00333, Revision 1.00, March 1997

[23] LeMans VX8 Carrier Board Programming Guide, Spectrum Signal Processing, Document Number 500-00330, Revision 2.00, November, 1997

[24] TMS340C40 Emulator Tips, TMS320 DSP Designer's Notebook, Texas Instrument, No. 32

[25] Updated VX8 software, www.spectrumsignal.com, Files 00200377.exe and 00203630.exe (password protected)

*RL-TR-95-31 is Distribution Authorized to U.S. Government Agencies and their Contractors; Critical Technology. 
[26] LeMans VX8 Carrier Board Installation Guide, Spectrum Signal Processing, Document Number 500-00328, Revision 1.01, August 1997

[27] Lemans VX8 Carrier Board Installation Guide, Spectrum Signal Processing, Document Number 500-00328, Revision 2.00, November 1997

[28] TMS340C40 Emulator Tips, TMS320 DSP Designer's Notebook, Texas Instrument, No. 32

[29] Private email message from Mr. Mourad Chergui, Spectrum Signal Processing Inc., August 20, 1999

[30] Ho, K.C., Chan, Y. T., Inkol, R., "A Digital Quadrature Demodulation System," AES32-4, October, 1996, 1218-1226

[31] Rice, D. W. and Wu, K. H., " Quadrature Sampling with high dynamic range,", AES18, 4 Nov 1982), 726-739

[32] Ward, H. R., "An optimum filter for direct A/D conversion," AES-27, 56 (Nov. 1991), 883-886

[33] Rader, C. M., " A simple method for sampling in-phase and quadrature components", AES-20, 6 (Nov 1984), 821-824

[34] Mitchell, R. I., " Creating complex signal samples from a band-limited real signal", AES-25, 3 (May 1989), 425-427 


\section{APPENDIX A. TERMS AND DEFINITIONS}

$\mathrm{A}_{\mathrm{e}}(\theta, \phi)=$ Receive antenna aperture toward elevation $\phi$ and azimuth $\theta\left(\right.$ meter $\left.^{2}\right)$

$A_{c o l}=$ effective aperture of a column array

$A_{\text {row }}=$ effective aperture of the row dimension created by digital beamforming

$B_{\text {coh }}=$ noise bandwidth of a coherent processing interval $\cong \frac{L_{\text {wtr }_{n}} \mathrm{~B}_{\mathrm{s}}}{n_{\text {beam }}}(\mathrm{Hz})$

$B_{n}=$ noise bandwidth of analog receiver $(\mathrm{Hz})$

$B_{s}=$ bandwidth of signal $\leq \mathrm{B}_{\mathrm{n}}(\mathrm{Hz})$

$\mathrm{DC}=$ duty cycle $=\frac{\tau_{\mathrm{u}}}{\mathrm{T}_{\mathrm{pri}}}$

$\mathrm{F}_{\mathrm{t}}^{2}(\theta, \phi)=$ transmit propagation pattern

$\mathrm{F}_{\mathbf{r}}^{2}(\theta, \phi)=$ receiver propagation pattern

$\mathrm{G}_{\mathrm{pc}}=$ pulse compression $\mathrm{S} / \mathrm{N}$ gain $=\frac{L_{w t r} s}{L_{w t r_{n}}} \mathrm{~B}_{\mathrm{s}} \tau_{u} \approx \frac{\tau_{\mathrm{u}}}{\tau_{c}}$

$G_{r}=$ Receiver amplication

$G_{t}(\theta, \phi)=$ Transmit gain at azimuth $\theta$ and elevation $\phi$ relative to the maximum gain at $G_{t}(0,0)$

$L_{\text {wta }}=$ weighting loss in azimuth beamforming

$\mathrm{L}_{\mathrm{e}}=$ environment losses

$L_{c o l}=$ loss within a column array

$L_{\text {comp }}=$ signal loss in compensation network

$\mathrm{L}_{\text {conp }}=$ signal - to - noise loss in compensation network

$\mathrm{L}_{\mathrm{p}}=$ signal processing losses

$\mathrm{L}_{\mathrm{r}}=$ receiver losses

$\mathrm{L}_{\mathrm{t}}=$ transmit losses

$L_{w t a_{\mathrm{n}}}=$ noise loss of azimuth beamforming weighting

$L_{w t a_{s}}=$ signal loss of azimuth beamforming weighting

$L_{w t a}=$ signal - to - noise loss of azimuth beamforming weighting

$L_{w t p_{\mathrm{n}}}=$ noise loss of Doppler filter weighting

$L_{w t p_{\mathrm{S}}}=$ signal loss of Doppler filter weighting

$L_{w t p}=$ signal - to - noise loss of Doppler filter weighting 
$L_{w t r}=$ noise loss of pulse compression weighting

$L_{w t r}=$ signal loss of pulse compression weighting

$L_{w t r}=$ signal - to - noise loss of pulse compression weighting

$n_{\text {beam }}=$ number of pulses per beam

$N_{c o l}=$ number of columns

$N_{p}=$ number of pulses coherently integrated

$P_{t}=$ Transmitter Peak Power $=$ Average power within the pulse (watts)

$\bar{P}_{t}=$ average transmit power $=(D C) P_{t}$ (watts)

$Q=$ match filter response to a single pulse

$R_{r}=$ Range from the receiver to the target (meters)

$\mathrm{R}_{\mathrm{t}}=$ Range from the transmitter to the target (meters)

$S=$ average signal power within the pulse

$S(\omega)=$ signal voltage spectrum (italics used to distinguish from signal power )

$\mathrm{T}_{\text {beam }}=$ observation time per beam $=\mathrm{n}_{\text {beam }} \mathrm{T}_{\text {pri }}$ (seconds)

$\mathrm{T}_{\mathrm{S}}(\theta, \phi)=$ System noise temperature in elevation $\phi$ and azimuth $\theta\left({ }^{\circ} \mathrm{K}\right)$

$\mathrm{T}_{\text {pri }}=$ pulse repetition interval (sec)

$\mathrm{T}_{\text {sc }}=\frac{\mathrm{T}_{\text {beam }} \Psi}{\Omega}=$ time to scan solid angle $\Psi(\mathrm{sec})$

$\mathrm{u}(\mathrm{t})=$ transmit waveform

$\chi\left(\tau, \omega_{d}\right)=\int_{-\infty}^{\infty} u(x) u^{*}(\tau+x) e^{j \omega^{x}} d x / \chi_{n}=$ normalized ambiguity function of the burst waveform where $\tau$ and $\omega_{d}$ are offsets from the peak of the burst matched filter response.

$\chi_{n}=\int_{-\infty}^{\infty} n(x) u^{*}(\tau+x) e^{j \omega} d^{x} d x=$ burst matched filter response to noise

$\phi=$ elevation angle

$\theta=$ azimuth angle

$\eta_{a}=$ antenna efficiency (uniform weighting)

$\kappa=$ Boltzmann's constant $=1.38 * 10^{-23}$ watt - second $/{ }^{\circ} \mathrm{K}$

$\sigma_{\mathrm{t}}(\beta, \varphi, \gamma)=$ Target RCS at bistatic angle $\beta$ and aspect $(\varphi, \gamma)\left(\right.$ meter $^{2}$ )

$\tau_{u}=$ uncompressed pulsewidth (sec)

$\tau_{\mathrm{c}}=$ compressed pulsewidth (sec)

$\Omega_{t}=$ solid angle of transmit beam (steradians)

$\Psi_{t}=$ solid angle to be searched by transmitter (steradians) 


\section{APPENDIX B}

\section{LeMans}

Octal TMS320G4x VXIbus Master Board

The LeMrens io a VXIbus board based on Toxas Instruments TMS3z OCEx DSPs. The LolMans fentres two ch-board 60 MIIt THS320C10 DSP, fur a base 120 MFLOP DSP sub-system witich supports shaced mernory VXTbura mexted ave, sond the HP Local Bur. Processing pothex end memory capadty can be increased to 840 MFLOPS by populating the str avallable TIM-40 mochuls sites with Spectrumis compe honsive range of TTMV modules. The LeNans boets sir TIM-40 modulo sites to accommodate a combination of single $\sigma^{\circ}$ doublowidth TIM-40 mondules with SRAM, EDRAM, DRAM, or WO

\section{VXabus Interface}

The LeNans is a C stro VXI boand supporting

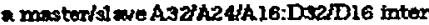
faco. Aby 'C4O or 'CA4 can become VXIbus master to road ar wite over the VXIbus

HP Local Bus Support

The LeMans surports Hewiett-Packerd's Local Bus. The LaMnns allows $60 \mathrm{MBy}$ tes/s tranofers through the shared bus to the globel SRAM of tho embedded ' $\mathrm{CeO}$.

A DMA broadcast write fo ature from the HF Local Bus to the DSPa allow the same block of I/O data to bo troaderst to each 'C4O's globel SRAM. This feature is extremely useful th ovorlap mocesstag applications. During a long HP Local Bus DMA transfor, embetded C $C 10$ may suspoend the tronsfor, servico the VIIbus interrupts, then resume tho HP Lacal Bus DMA transfor

\section{Shared Mamory}

The LeMrons apports a shared bus archttec ture which allows the on-bonerd 'C40s, the TIM-40 modules, and the VXIbus interfince to access an optianal bank of on-bosed giobal shared DRAM. Spectrum provides options for $0,4,8,32$, and 64MB of one watt-state DRAMI The shared memory architecture slso sllows the global SRAM resources avaliahle on-board and on the TIM-10 modules to be accessed firectly by any other C4O DSP. Esch ombed ded "C40 has access to 1 or 4 MBytos of Iero woit-state SRAM divided equally on its Global snd Loend Busses.
Front Panel interfaces

Eight buffered ' $C_{4} \times$ commumication ports are routed to the front panal for communica. tion with other 'C4x communication parts on bourd or on other VXIbus modules. The remaining ' $C 4 x$ communication ports aro interconnected on-boand to provtde optimm interprocessor commomications between the dght 'C40s. The front panel has two RS-232 sorial ports, an application-specific connector for TIM-10 modules supporting ITO, and LEDs for power and status infication.

\section{Software, Debuggers, and Debugging}

The LolMans has JTAG input and output conmectors for use with TTs or Spectrum's XDS debugger. The Lolkons board also has a Tost Bus Controller [TBC. Acossible from the VXIbus, the TBC allows debugaing from WhiNT-based VXIbus re source managers. Device thvor support is also avallable va VISA or SICL for resource managers bosting operating system such ses HP-UX and Windous. Spectrumsepports many more DSP tools based on the C40/CA4 stich as optimizod signal procesoing Itrourtes and DSP operating systoms.

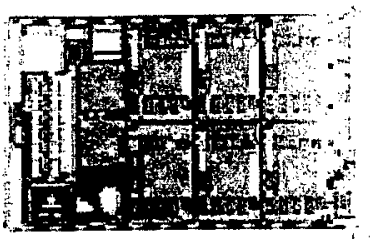

- ato MrLOPS UXubus Mastar/Slave DSP board

- Two enbedded 60 MHII TMS320C40 DSPS wh support for 6 mine-40 sitas

- HP Loeal Bus supports HP's Uxbus Yo hoands

- Eroadcast wite from VXlbus and HP Local Bus

- 4 to CMME or DRAM shargd with Walbus and all TM-10 sites

- Each -Geo can read and wrte to any 'C40's Global memory (1 wes within a page)

- Elght buffered front panel comm ports

- Single-słot vxtous c-sizecompilant

- Drher support for HP-UX and Mindows "9s

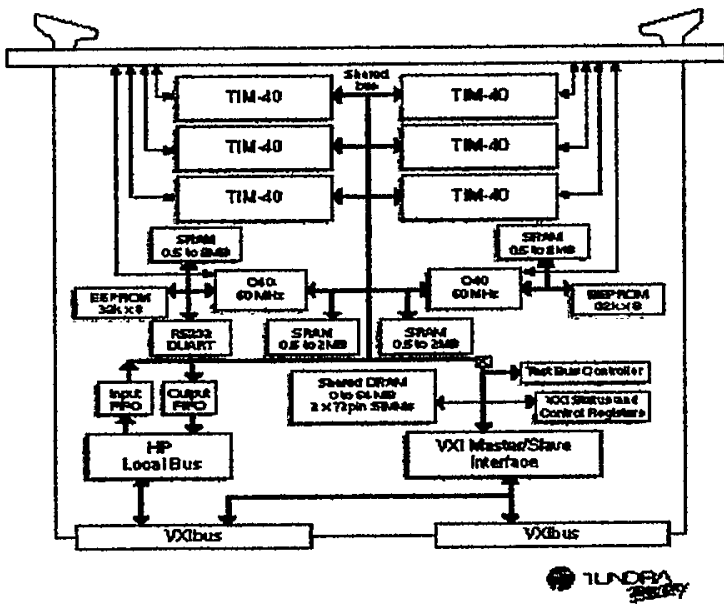

-C4x-bosed carrier boends DO NOT include TIM modules 